\title{
Reduction of Forward Difference Operators in Principal G-bundles
}

\author{
Ana Casimiro ${ }^{1}$, César Rodrigo ${ }^{2, *}$ \\ ${ }^{1}$ CMA - Centro de Matemática e Aplicações, Departamento de Matemática, Faculdade de Ciências e Tecnologia, \\ Universidade Nova de Lisboa, Quinta da Torre 2829-516 Caparica, Portugal \\ ${ }^{2}$ CMAF-CIO - Centro de Matemática, Aplicações Fundamentais e Investigação Operacional, \\ CINAMIL, Academia Militar, Av. Conde Castro Guimarães, 2720-113 Amadora, Portugal
}

\begin{abstract}
Retraction maps on Lie groups can be successfully used in mechanics and control theory to generate numerical integration schemes, for ordinary differential equations with a variational origin, recovering at the same time a discrete version of the energy and symplectic structure conservation properties, that are characteristic of smooth variational mechanics. The present work fixes the specific tool that plays in gauge field theories the same role as retraction maps on geometric mechanics. This tool, the covariant reduced projectable forward difference operator, can be used for a covariant discretization of the main elements of a variational theory: the jet bundle, the Lagrangian density and the associated action functional. Particular interest is dedicated to the trivialized formulation of a gauge field theory, and its reduction into a theory where fields are given as principal connections and $H$-structures. Main characteristics of the presented method are its covariance by gauge transformations and the commutation of the discretization and the reduction processes.
\end{abstract}

Keywords Discretization, Reduction, Variational Principles, Euler-Poincaré Equations, Forward Difference Operators

AMS 2010 subject classifications 39A12, 49M25, 37J15, 65D25, 65D30

DOI: $10.19139 /$ soic.v6i1.468

\section{Introduction}

Classical numerical algorithms formulated on a linear space can, in many cases, be generalized to an arbitrary manifold, if we fix some operator that allows to measure the difference between two elements of the manifold as an object on a linear space. A second operator, inverse to this one, allows to determine the translation of a point by one of these linear elements. With these simple tools, several classical numerical algorithms, originally developed for affine spaces, can be extended to new numerical algorithms on manifolds [1]. The choice of the mechanism to compute such linear differences determines which numerical properties of the classical algorithm are maintained by the new one on manifolds, and is also relevant for the behaviour of the algorithm with respect to geometrical transformations on the manifold.

A particular successful example of these ideas is obtained in geometric mechanics, where the linear difference $\Delta(g, h) \in \operatorname{Lie} G$ between two points on a Lie group $G$ is determined by $\tau(\Delta(g, h))=g^{-1} h$ using $\tau$ : Lie $G \rightarrow G$ the exponential, the Cayley transform, or any other choice of what is called a retraction map on a Lie group [26]. Using this idea, the linear differences are preserved, $\Delta(t g, t h)=\Delta(g, h)$, if one translates the pair of points on $G$ using left multiplication by any other group element $t \in G$. As a consequence, one can devise tools that associate an element of a discrete variational theory to each element of a smooth variational theory, preserving at the same time many of the symmetries available in the smooth formulation. One can then explore the consequences, in the

*Correspondence to: César Rodrigo (Email: crodrigo@geomat-pt.com). Academia Militar, Av. Conde Castro Guimarães, 2720-113 Amadora, Portugal.

ISSN 2310-5070 (online) ISSN 2311-004X (print)

Copyright (C) 2018 International Academic Press 
discrete formulation, of the existence of symmetries in the original smooth objects. In particular, to obtain discrete conservation laws and discrete reduction schemes, resembling those results existing in the smooth theory.

A natural step forward is to extend this kind of ideas to field theories. In Lagrangian gauge field theories, where a subgroup $H \subseteq G$ acts by symmetries of the Lagrangian, it is known that classical Euler-Lagrange equations can be reduced as Euler-Poincaré equations, where dependent variables are a principal connection and an $H$ structure [14]. Its applications appear in several areas. Classical gauge fields with symmetry breaking considered in theoretical physics can be described as solutions of these equations. Many geometrical field theories consider energy minimizing mappings or immersions from a manifold $X$ into another one (for example harmonic maps). In the case that the immersion is into a Lie group $G$ or an homogeneous space $G / H$ (which is a frequent situation), fields can be seen as sections of a trivial principal bundle $X \times G$ or an $H$-reduction of such a section, fitting in our model. Euler-Poincaré equations in this situation are necessary conditions for a field to minimize an action functional described by a first order Lagrangian. In natural sciences such minimization principles are also common for the determination of an equilibrium state or dynamics of a certain continuous medium with or without microstructure. These theories include molecular strands, liquid crystals, fluid flows, or magnetohydrodynamics for example [22].

Discrete formulations of the notion of principal connection have already been explored in geometric mechanics $[24,32]$. There exist approaches to discrete reduced field theories in certain particular cases. The simplest case is that of a field with values on a Lie group (that is, a field that is a section of a trivial principal bundle). Another approach is to consider a discrete field theory as a discrete mechanical theory, where the configuration at a fixed time is composed by many degrees of freedom, and to apply then the available results from discrete geometric mechanics. There has been a certain amount of works exploring these situations [18, 23, 25, 39, 40]. Many of these formulations are purely discrete versions of the smooth theory, and don't explore the several alternative ways that lead from a smooth field theory to a discrete one. This article takes the most general gauge field theory (not necessarily on a trivial bundle) and explores a general covariant mechanism to discretise a variational principle and its possible reduction by a symmetry group, extending to principal bundles ideas that have been originally formulated for the reduction to discrete Euler-Poincaré equations in mechanics on a Lie group.

Our work starts in section 2 with a review of the notions of retraction maps and finite difference operators on manifolds, the relation between them, and how translation-invariant retraction or finite difference maps can be seen as reduced retraction maps. Section 3 reminds how reduced retraction maps on Lie groups render a covariant discretization in variational theories of geometric mechanics. Section 4 explores smooth variational field theories on principal $G$-bundles and its reduction when a subgroup $H \subseteq G$ acts as symmetries of the Lagrangian density. Section 5 describes how a forward difference operator on the principal bundle can be used to relate the main objects of the smooth variational theory with its discrete analogues. Section 6 explores Ehresmann's groupoid associated to a principal bundle as the natural discrete counterpart of the bundle of principal connections. Section 7 explores the notion of covariant forward difference operator and how it is equivalent to a notion of reduced forward difference operator. Section 8 introduces the discrete connection space, related with the smooth bundle of principal connections through a corresponding reduced forward Jacobi operator (that can be generated by a reduced forward difference operator).

To make the article self-contained we include (sections 2, 3 and partly 4) a description of the basic notions and properties of difference operators and retractions, a description of the particular case of discretization of these theories in mechanics, leading to discrete Euler-Poincaré equations, and the needed machinery for gauge field theories and its reduction. Several useful objects in principal bundle theory, like Ehresmann's gauge groupoid, are also presented in the work. However we assume the reader has a good knowledge of differential calculus and geometry on fibered manifolds, in particular knowledge of the usual notation in this area.

Our main results, besides presenting and relating all these areas, are described next:

We give a trivialization, in terms of principal connections, of the jet bundle and of its $H$-reduction (proposition 4.5 and corollary 4.1), introducing and describing the properties of forward difference operators on fibered manifolds, and of its associated objects, the $n$-Jacobian map, which allows to discretize volume forms, and the Forward Jacobi operator, which identifies the jet bundle with a multi-point manifold (Theorem 5.1) allowing to discretize lagrangian densities (definition 5.12). We identify in which cases this discretization of the jet bundle is 
coherent with a limit process (counter-example 5.1 and theorem 5.2) and prove the covariance of the discretization mechanism. The discretization mechanism is covariant, in the sense given by Proposition 5.3.

We introduce then the reduction process. We obtain in theorem 6.2 a description of the tangent space of Ehresmann's gauge groupoid bundle and its possible trivializations using Atiyah's bundle (corollary 6.1), determine a notion of parallelism and how it may be generated from a principal connections if a forward difference operator is fixed (theorem 6.4). The particular case of forward difference operators that are covariant for the natural action of the Lie group lead to the notion of reduced forward difference operators and its properties (proposition 7.2). We prove the existence and describe the structure of a projectable, faithful, reduced forward difference operators on a bundle, fixing a forward difference operator on the base manifold, a parallelism on the principal bundle, and a retraction map on the gauge bundle (theorem 7.3).

Finally, we study the reduction of variational formulations of gauge field theories. We show that for the reduced theory, a reduced forward difference operator leads to a discretization process, identifying the connection bundle of the theory with a discrete version, and the $H$-reduced jet bundle with another discrete version, with the desired behaviour in the limit case for multi-points coalescing to a diagonal point (theorem 8.3, theorem 8.4). This allows to introduce a discretization of $H$-reduced lagrangian densities (definition 8.3). This object maintains all the symmetries of the smooth object, if the RFD operator admits these symmetries, in such a way that both processes of $H$-reduction and of discretization (in the smooth and in the discrete formulations) commute (in the sense of Figure 2 and theorem 8.5).

The present work shows that a single object, the faithful, projectable, reduced forward difference operator, represents a central element in the discretization of variational gauge field theories, leading to mechanisms that relate reduced or un-reduced, smooth or discrete gauge fields, in a compatible way, and preserving the available symmetries.

\section{Retraction maps and Forward difference operators}

An ordinary differential equation (ODE) on a Lie group $G$ has the fundamental form $\dot{g}(t)=g(t) \cdot \xi(t, g(t))$, for some mapping $\xi: \mathbb{R} \times G \rightarrow \operatorname{Lie} G$ determining the ODE. In the case that this mapping has a constant value $\xi \in$ Lie $G$, the differential equation admits left translations as symmetries and the trajectory $g(t)=g_{0} \cdot \exp \left(\left(t-t_{0}\right) \xi\right)$ is a solution for initial conditions $g\left(t_{0}\right)=g_{0} \in G$. This trajectory generated by the exponential map plays, on a Lie group, the role played by line segments in linear spaces. In this sense, ODEs on Lie groups can be numerically solved translating numerical schemes from the vector space Lie $G$ to the nonlinear space $G$ by means of the exponential map, as done in Crouch-Grossman, or Runge-Kutta-Munthe-Kaas methods [17, 26]. Several optimization algorithms on manifolds rely also on some Lie group exponential map [2, 20]. Moreover, these ideas can be easily generalized (and the numerical schemes improved) substituting the exponential map by the more general notion of reduced retraction maps on Lie groups.

Definition 2.1

We call reduced retraction map on a Lie group $G$ any injective local diffeomorphism $\tau: U_{0} \rightarrow G$ defined on an open neighborhood $U_{0} \subset \operatorname{Lie} G$ of the null element $0 \in \operatorname{Lie} G$, such that

$$
\tau(0)=e, \quad \mathrm{~d}_{0} \tau=\operatorname{Id} \in \operatorname{End}(\operatorname{Lie} G)
$$

where $e \in G$ is the unit element of $G$ and in $\mathrm{d}_{0} \tau: T_{0} \operatorname{Lie} G \rightarrow T_{e} G$ we use the identifications $T_{e} G \simeq \operatorname{Lie} G$, Lie $G \simeq T_{0} \operatorname{Lie} G$.

From this definition, a retraction map is a diffeomorphism from $U_{0}$ to some open neighbourhood $U_{e}=\tau\left(U_{0}\right) \subset$ $G$ of the of the unit element $e \in G$. In this sense, they have been also called generalized coordinates. They have proved to be a key element, to derive numerical methods associated to Euler-Lagrange equations of a discrete variational problem, arising in geometric mechanics, with Noether current conservation properties in the presence of symmetries $[5,8,27,28,29,35]$. This approach has also been employed for the dynamics of continuous media [18]. This reduced retraction map notion on a Lie group is simply the reduced version in some quotient space of a 
more general notion of retraction map on any manifold. The origin of such retraction maps in numerical schemes on any manifold can be tracked back to [38], where it is used to generalize Newton's method for the problem of finding the zeros on a manifold for some $\mathbb{R}^{n}$-valued function. It is used in several other algorithms for optimization in manifolds [1].

Definition 2.2 (Adapted from [1, 2], originated in [38])

Consider a manifold $X$, its tangent bundle $\pi_{T X}: T X \rightarrow X$, and the zero section $z: x \in X \mapsto 0_{x} \in T X$.

A smooth retraction on a manifold $X$ is a smooth mapping $R: U_{z} \rightarrow X$ defined on an open subset $U_{z} \subset T X$ containing the image of the zero section, such that for each restriction $R_{x}: U_{x} \rightarrow X$ to the $x$-fiber $U_{x}=U_{z} \cap T_{x} X$ there holds:

1. $R_{x}\left(0_{x}\right)=x$

2. $\mathrm{d}_{0_{x}} R_{x}: T_{0_{x}}\left(T_{x} X\right) \rightarrow T_{x} X$ is the identity map

(using the natural identification $T_{v} V \simeq V$ for the vector $v=0_{x}$ in the vector space $V=T_{x} X$ )

We say the retraction is covariant for some smooth automorphism $\varphi: X \rightarrow X$, if $U_{z} \subset T X$ is invariant by the induced differential mapping $\mathrm{d} \varphi: T X \rightarrow T X$ and $R \circ \mathrm{d} \varphi=\varphi \circ R$.

In Newton's method [2], a retraction is usually chosen as a way to take small steps in the direction of a tangent vector of a manifold, allowing to generate a new point $\bar{x}=R_{x}\left(\delta_{x}\right) \in X$ from a (small enough) tangent vector $\delta_{x} \in U_{x} \subset T_{x} X$. A usual choice of retraction is the exponential on a Riemannian manifold, which solves the geodesic equation using $\delta \in T X$ as initial conditions. Alternative choices may be used to avoid the computational cost of determining the exact exponential in several situations.

For the case of a Lie group $X=G$, this definition of retraction map $R: U_{z} \rightarrow G$ (where $U_{z} \subset T G$ ) does not coincide with the previously given (reduced) notion as a mapping $\tau: U_{0} \rightarrow G$ (where $U_{0} \subset \operatorname{Lie} G$ ).

\section{Proposition 2.1}

For any reduced retraction map $\tau: U_{0} \rightarrow G$ on a Lie group $G$, the locally defined mappings $R_{g}=l_{g} \circ \tau$ 。 $\left(\mathrm{d}_{e} l_{g}\right)^{-1}: T_{g} G \rightarrow G$ generate a retraction map $R: U_{z} \rightarrow G$ on the manifold $G$ that is covariant for left translations $l_{h}: g \in G \mapsto h g \in G$. Conversely, any retraction map $R$ on the manifold $G$ covariant for left translations determines a reduced retraction map $\tau$ that generates $R$ (in the mentioned sense), on some open neighborhood $U_{z} \subset T G$ of the zero section.

Proof

Consider the natural isomorphism $G \times \operatorname{Lie} G \simeq T G$ that identifies $(h, \xi)$ with $\left(\mathrm{d}_{e} l_{h}\right)(\xi)$, for any $\xi \in T_{e} G=\operatorname{Lie} G$ (using the left translation morphism $l_{h}: g \in G \mapsto h g \in G$ ). With this isomorphism, the automorphism $\mathrm{d} l_{g}$ on $T G$ gets identified with $l_{g} \times \operatorname{Id}_{\operatorname{Lie} G}$ on $G \times \operatorname{Lie} G$, the projector $\pi_{T G}: T G \rightarrow G$ gets identified with the natural projector $\pi_{G}: G \times \operatorname{Lie} G \rightarrow G$, and the null section $z: G \rightarrow T G$ with the section (Id, 0): $G \rightarrow G \times \operatorname{Lie} G$.

Any reduced retraction map $\tau: U_{0} \rightarrow G$ naturally extends to $(g, \xi) \in G \times U_{0} \mapsto g \cdot \tau(\xi) \in G$. Using $G \times$ Lie $G \simeq T G$, we have a neighborhood $U_{z} \subset T G$ (identified with $\left.G \times U_{0}\right)$ and a smooth mapping $R: U_{z} \rightarrow G$ defined on fibers $U_{g}=U_{z} \cap T_{g} G=\left(\mathrm{d}_{e} l_{g}\right)\left(U_{0}\right) \subset T_{g} G$, for which $R_{g}=l_{g} \circ \tau \circ\left(\mathrm{d}_{e} l_{g}\right)^{-1}$ holds. Using $\tau(0)=e$, $\mathrm{d}_{0} \tau=\operatorname{Id}_{\text {Lie } G}$ there easily follows $R_{g}\left(0_{g}\right)=g, \mathrm{~d}_{0_{g}} R_{g}=\mathrm{Id}_{T_{g} G}$, hence $R$ is a retraction map. Moreover, this retraction map has the particularity that $R_{h g} \circ \mathrm{d}_{g} l_{h}=l_{h} \circ R_{g}$ on $U_{g}$ (therefore the retraction is covariant with respect to the left action morphisms of the group $G$ on itself).

Conversely, using $T G \simeq G \times \operatorname{Lie} G$, any retraction map $R: U_{z} \rightarrow G$ on the manifold $G$ (where $U_{z} \subset T G$ ) can be seen as a particular mapping $\bar{R}: \bar{U}_{z} \rightarrow G$ with $\bar{U}_{z} \subset G \times \operatorname{Lie} G$ and $\bar{R}(g, \xi) \in G$. In the case that the retraction map is covariant with respect to left translations, the domain $\bar{U}_{z}$ is invariant by left-translations on the component $G$, and has the form $\bar{U}_{z}=G \times U_{0}$ where $U_{0} \subset \operatorname{Lie} G$ is an open neighbourhood of $0 \in \operatorname{Lie} G$. Moreover, we get $\bar{R}(h g, \xi)=h \bar{R}(g, \xi)$ and this covariant retraction map takes the form $\bar{R}(g, \xi)=g \tau(\xi)$ for some mapping $\tau: U_{0} \rightarrow G$ for which $\tau(0)=e, \mathrm{~d}_{0} \tau=\mathrm{Id}$ (because $\mathrm{d}_{0_{g}} R_{g}=\mathrm{Id}: T_{g} G \rightarrow T_{g} G$ ). This implies that $\tau$ is a local diffeomorphism at 0 , and there exists a possibly smaller open neighbourhood of $0 \in \operatorname{Lie} G$ where $\tau$ is a reduced retraction map. There easily follows that this reduced retraction map generates $R$. 
A smooth retraction on any manifold $X$ also determines a smooth morphism $\tau_{R}=\left(\pi_{T X}, R\right): U_{z} \rightarrow X \times X$, defined on the open neighborhood $U_{z} \subset T X$ of the zero section $z: X \rightarrow T X$. The null section $z \subset T X$ is transformed onto the diagonal $d_{1}(X) \subset X \times X$, and any fiber $U_{x}=U_{z} \cap T_{x} X$ is transformed into an open neighborhood $\{x\} \times V_{x} \subset\{x\} \times X$ of the diagonal point $(x, x)$. As the diagonal and the fiber $\{x\} \times X$ are transversal submanifolds, we conclude that $\tau_{R}$ is a local diffeomorphism. Taking a restriction to a smaller neighborhood of the zero section, if needed, we may admit that $\tau_{R}$ is an injective local diffeomorphism, from $U_{z}$ onto an open neighbourhood $U_{d}=\tau_{R}\left(U_{z}\right) \subset X \times X$ of the diagonal section $d_{1}: x \in X \mapsto(x, x) \in X \times X$. Moreover, $\tau_{R}$ is a locally defined fibered mapping covering $\operatorname{Id}_{X}$, when one considers the bundle $\pi_{T X}: T X \rightarrow X$ and the bundle $\pi_{0}:\left(x_{0}, x_{1}\right) \in X \times X \mapsto x_{0} \in X$ (that we call the pair bundle associated to $\left.X\right)$. We have $\pi_{0} \circ \tau_{R}=$ $\pi_{T X}$. Taking the locally defined inverse bundle morphism $\tau_{R}^{-1}: U_{d}=\tau_{R}\left(U_{z}\right) \rightarrow T X$, we obtain a particular instance of a finite difference map $\Delta: X \times X \rightarrow T X$, as defined below.

Definition 2.3 (Adapted from [31])

A finite difference map $\Delta$ is an injective local diffeomorphism $\Delta: U_{d} \rightarrow T X$ defined on an open neighbourhood $U_{d} \subset X \times X$ of the diagonal section $d_{1}: x \in X \mapsto(x, x) \in X \times X$ of the pair bundle, which satisfies the following:

$$
\Delta(x, x)=0_{x} \in T_{x} X, \quad \forall x \in X
$$

We say a difference map $\Delta$ is covariant with respect to some automorphism $\varphi: X \rightarrow X$, if $U_{d}$ is invariant by $\varphi \times \varphi$ and $\Delta \circ(\varphi \times \varphi)=\mathrm{d} \varphi \circ \Delta$.

Again, the condition that $\Delta$ is an injective local diffeomorphism indicates that it is a diffeomorphism onto some open neighborhood $U_{z} \subset T X$ of the zero section.

Observe that $\Delta\left(x_{0}, x_{1}\right) \in T X$ is a tangent vector at a point $\kappa\left(x_{0}, x_{1}\right) \in X$, for some smooth mapping $\kappa=$ $\pi_{T X} \circ \Delta: X \times X \rightarrow X$. We shall be interested in finite difference maps, such that $\pi_{T X} \circ \Delta=\pi_{0}:\left(x_{0}, x_{1}\right) \in$ $X \times X \mapsto x_{0} \in X:$

\section{Definition 2.4}

We call forward difference (FD) operator on a manifold $X$, any finite difference map $\Delta: U_{d} \rightarrow T X$ (in the sense of Definition 2.3) covering $\operatorname{Id}_{X}$ for the first projector $\pi_{0}:\left(x_{0}, x_{1}\right) \in X \times X \mapsto x_{0} \in X$ and the natural projector $\pi_{T X}: T X \rightarrow X$ (that is, $\left.\pi_{T X} \circ \Delta=\pi_{0}\right)$.

In a similar way, a backward difference operator is determined demanding that $\pi_{T X} \circ \Delta$ coincides with the projector $\pi_{1}:\left(x_{0}, x_{1}\right) \mapsto x_{1}$ on its domain of definition. Composition with $\left(x_{0}, x_{1}\right) \mapsto\left(x_{1}, x_{0}\right)$ transforms backward into forward difference operators. More general finite difference maps and its properties are studied in $[31,35]$.

For any retraction $R$, computing a local inverse of $\tau_{R}=\left(\pi_{T X}, R\right)$ leads to a FD operator $\Delta$. If the retraction is covariant for $\varphi$, also $\Delta$ will be covariant for $\varphi$. As a consequence, on Lie groups, there is a relation leading from a reduced retraction map $\tau: U_{0} \subset$ Lie $G \rightarrow G$ to a covariant retraction $R: U_{z} \subset T G \rightarrow G$ and then to a covariant FD operator $\Delta: U_{d} \subset G \times G \rightarrow T G$ (inverse of $\tau_{R}=\left(\pi_{T G}, R\right)$ ). The particular conditions imposed on a retraction map indicate that the differential of $\tau_{R}$ on the $\pi_{T X}$-vertical vectors, at the zero elements $0_{x} \in T_{x} X$ should be the identity mapping, if we use the appropriate identifications. The same can be said about the differential of $\tau_{R}^{-1}$ on the $\pi_{0}$-vertical vectors, at the diagonal elements $(x, x) \in X \times X$, with the appropriate identifications that we describe next.

For any local $X$-bundle morphism $D: X \times X \rightarrow T X$, between the bundles $\pi_{0}: X \times X \rightarrow X$ and $\pi_{T X}: T X \rightarrow$ $X$, defined on a neighborhood of the diagonal, demanding that $D$ is a local diffeomorphism at $\left(x_{0}, x_{1}\right)$ is equivalent to demanding that the vertical component (for the $\pi_{0}$-fibration) of its differential $\mathrm{d}_{\left(x_{0}, x_{1}\right)} D: V_{\left(x_{0}, x_{1}\right)}^{\pi_{0}}(X \times X) \rightarrow$ $V_{D\left(x_{0}, x_{1}\right)}^{\pi_{T X}}(T X)$ is a linear isomorphism.

We may observe that there exist natural identifications $V_{\left(x_{0}, x_{1}\right)}^{\pi_{0}}(X \times X) \simeq T_{x_{1}} X$ and $V_{\delta_{x}}^{\pi_{T X}}(T X) \simeq T_{x} X$. For any $X$-bundle morphism $D: X \times X \rightarrow T X$, the vertical component of the differential $\mathrm{d}_{\left(x_{0}, x_{1}\right)} D$ can be then 
represented using a linear morphism $V D_{\left(x_{0}, x_{1}\right)}: T_{x_{1}} X \rightarrow T_{x_{0}} X$, that makes the following diagram commutative:

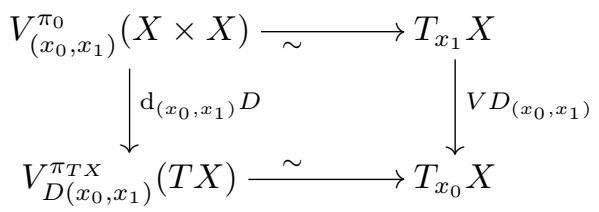

The particular hypothesis $\mathrm{d}_{0} \tau=\mathrm{Id}$ ( or $\mathrm{d}_{0_{x}} R_{x}=\mathrm{Id}$ ) imposed to retractions and reduced retraction maps lead to particular FD operators, that we characterize next:

Definition 2.5

A FD operator $\Delta$ is called faithful, if at any point of the diagonal, the vertical component of its differential $V \Delta_{(x, x)}: T_{x} X \rightarrow T_{x} X$ is the identity map $\operatorname{Id}_{T_{x} X}$ (using the natural identifications $V_{\left(x_{0}, x_{1}\right)}^{\pi_{0}}(X \times X) \simeq T_{x_{1}} X$ and $\left.V_{\delta_{x}}^{\pi_{T} X} T X \simeq T_{x} X\right)$.

Example 2.1

- For an affine space $X$ modelled over a vector space $\vec{X}$, a faithful FD operator can be defined by $\Delta\left(x_{0}, x_{1}\right)=$ $\overrightarrow{x_{0} x_{1}} \in \vec{X} \simeq T_{x_{0}} X$, where we use the classical notation $x_{0}+\overrightarrow{x_{0} x_{1}}=x_{1}$ and the natural identification $T_{x} X \simeq \vec{X}$, at any point $x \in X$.

In particular, $\left(t_{0}, t_{1}\right) \in \mathbb{R} \times \mathbb{R} \mapsto\left(t_{1}-t_{0}\right)(\partial / \partial t)_{t_{0}} \in T \mathbb{R}$ is a faithful FD operator.

- For any manifold, using any $X$-bundle injective local diffeomorphism $D: U_{d} \rightarrow T X\left(U_{d} \subset X \times X\right.$ open subset containing the diagonal), the local diffeomorphism condition ensures that $V D_{(x, x)}: T_{x} X \rightarrow T_{x} X$ is invertible and one may derive a FD operator $\Delta$ defined on $U_{d}$ as $\Delta\left(x_{0}, x_{1}\right)=D\left(x_{0}, x_{1}\right)-D\left(x_{0}, x_{0}\right)$, and a faithful FD operator just by taking $\Delta\left(x_{0}, x_{1}\right)=\left(V D_{\left(x_{0}, x_{0}\right)}\right)^{-1}\left(D\left(x_{0}, x_{1}\right)-D\left(x_{0}, x_{0}\right)\right)$.

- On a Lie group $G$, considering the exponential map exp: $T_{e} G \rightarrow G$, we may determine a smooth map $\tau_{\exp }: T G \rightarrow G \times G$ using $\tau_{\exp }\left(\delta_{g}\right)=\left(g, g \cdot \exp \left(\left(\mathrm{d}_{e} l_{g}\right)^{-1}\left(\delta_{g}\right)\right)\right)$, for each $\delta_{g} \in T_{g} G$. This mapping transforms the null vector $\delta_{g}=0_{g} \in T_{g} G$ into the diagonal point $(g, g) \in G \times G$. Moreover, we observe that $\left(\mathrm{d}_{e} l_{g}\right)^{-1}\left(0_{g}\right)=0_{e},\left(\mathrm{~d}_{e} l_{g}\right)^{-1}: T_{g} G \rightarrow T_{e} G$ is linear, therefore its differential $T_{g} G \simeq T_{0_{g}}\left(T_{g} G\right) \rightarrow$ $T_{0_{e}}\left(T_{e} G\right) \simeq T_{e} G$ coincides with $\left(\mathrm{d}_{e} l_{g}\right)^{-1}$ itself, and $\mathrm{d}_{0_{e}}$ exp: $T_{e} G \simeq T_{0}\left(T_{e} G\right) \rightarrow T_{e} G$ is the identity map. Hence, $\mathrm{d}_{0_{g}} \tau_{\exp }$, restricted to vectors $\delta_{0} \in V_{0_{g}}^{\pi_{T G}}(T G)$ (that is, tangent vectors at $0_{g}$ of the fiber $\pi_{T G}^{-1}(g)=$ $\left.T_{g} G \subset T G\right)$ determines the identity map $A_{g} \in T_{g} G \mapsto \mathrm{d}_{e} l_{g} \circ \mathrm{d}_{0_{e}} \exp \circ\left(\mathrm{d}_{e} l_{g}\right)^{-1}\left(A_{g}\right)=A_{g} \in T_{g} G$. We conclude that $\mathrm{d}_{0_{g}} \tau_{\exp }: T_{g} G \rightarrow V_{(g, g)}^{\pi_{0}}(G \times G)=T_{g} G$ is the identity map and consequently any local inverse $\Delta: G \times G \rightarrow T G$ of $\tau_{\text {exp }}$ determines a faithful FD operator on the Lie group $G$.

- On a Riemannian manifold $X$, we may determine an open subset $U \subset T X$ containing the null vectors $0_{x} \in T_{x} X$, where the Riemannian exponential is well defined, exp: $U_{0} \rightarrow X$. This Riemannian exponential has the property that for each $A_{x} \in T_{x} X$ and for values $\epsilon \geq 0$ such that $\epsilon A_{x} \in U_{0}$, the trajectory $\exp \left(\epsilon A_{x}\right)$ is a minimal geodesic on $X$, with tangent vector $A_{x}$ for $\epsilon=0$. The Riemannian exponential is then a local diffeomorphism defined on a neighborhood of the null section, such that $\exp 0_{x}=x \in X$ for the null element $0_{x} \in T_{x} X$, and whose differential at $0_{x}$ is $\operatorname{Id}_{T_{x} X}: T_{x} X \rightarrow T_{x} X$. Consequently, $\tau_{\text {Rie }}: \delta_{x} \in T_{x} X \mapsto\left(x, \exp \delta_{x}\right) \in X \times X$ is a local diffeomorphism, transforming the null section into the diagonal on $X \times X$. A faithful FD operator is obtained using any local inverse of the mapping $\tau_{\text {Rie }}$.

In the presence of faithful FD operators we may generate curve segments with analogous properties to line segments in affine geometry, as shown below:

Theorem 2.2

Consider any faithful FD operator $\Delta: U_{d} \rightarrow T X$, where $\Delta\left(U_{d}\right) \subset T X$ is a star-convex neighbourhood of the zero section (that is, an open subset containing $\epsilon \delta_{x}$ for each $\delta_{x} \in \Delta\left(U_{d}\right)$ and each $\epsilon \in[0,1]$ ). 
There exists a unique smooth mapping $\alpha:\left(x_{0}, x_{1}, \epsilon\right) \in U_{d} \times[0,1] \mapsto \alpha_{x_{0} x_{1}}(\epsilon) \in X$ satisfying

$$
\Delta\left(x_{0}, \alpha_{x_{0} x_{1}}(\epsilon)\right)=\epsilon \Delta\left(x_{0}, x_{1}\right)
$$

The mapping $\alpha$ determines curve segments $\left\{\alpha_{x_{0} x_{1}}(\epsilon)\right\}_{0 \leq \epsilon \leq 1} \subset X$ joining $x_{0}$ at $\epsilon=0$ to $x_{1}$ at $\epsilon=1$, with tangent vector $\Delta\left(x_{0}, x_{1}\right) \in T_{x_{0}} X$ at $\epsilon=0$.

Moreover, for each point $x_{c}=\alpha_{x_{0} x_{1}}(c)$ there holds $\alpha_{x_{0} x_{c}}(\epsilon)=\alpha_{x_{0} x_{1}}(c \epsilon)$.

Proof

When we apply the inverse mapping $\Delta^{-1}$ to (1) we get:

$$
\left(x_{0}, \alpha_{x_{0} x_{1}}(\epsilon)\right)=\Delta^{-1}\left(\epsilon \Delta\left(x_{0}, x_{1}\right)\right)
$$

Consider the natural projectors $\pi_{0}:\left(x_{0}, x_{1}\right) \mapsto x_{0}, \pi_{1}:\left(x_{0}, x_{1}\right) \mapsto x_{1}$ and $\pi_{T X}: T X \rightarrow X$. We know $\pi_{T X}\left(\epsilon \Delta\left(x_{0}, x_{1}\right)\right)=\pi_{T X}\left(\Delta\left(x_{0}, x_{1}\right)\right)=x_{0}$, therefore the smooth mapping $\alpha$ defined as $\alpha_{x_{0} x_{1}}(\epsilon)=\pi_{1} \circ$ $\Delta^{-1}\left(\epsilon \Delta\left(x_{0}, x_{1}\right)\right)$ is smooth and satisfies (1).

From the definition and $\Delta\left(x_{0}, x_{0}\right)=0$ it is clear that $\alpha_{x_{0} x_{1}}(0)=x_{0}, \alpha_{x_{0} x_{1}}(1)=x_{1}$ and taking derivatives in (1) at $\epsilon=0$ we have

$$
\left.\left(\mathrm{d}_{\left(x_{0}, x_{0}\right)} \Delta\right)\left(0,(d / d \epsilon)_{0} \alpha_{x_{0} x_{1}}(\epsilon)\right)=(d / d \epsilon)_{0}\left(\epsilon \Delta\left(x_{0}, x_{1}\right)\right)\right)=\Delta\left(x_{0}, x_{1}\right)
$$

which, taking into account $\left(\mathrm{d}_{\left(x_{0}, x_{0}\right)} \Delta\right)=\mathrm{Id}$ for a faithful FD operator, shows that the tangent vector at $\epsilon=0$ of the trajectory $\alpha_{x_{0} x_{1}}$ is $\Delta\left(x_{0}, x_{1}\right)$.

The trajectory $\alpha_{x_{0} x_{1}}$ is characterized by (1). Hence for $x_{c}=\alpha_{x_{0} x_{1}}(c)$ there holds:

$$
\Delta\left(x_{0}, \alpha_{x_{0} x_{c}}(\epsilon)\right)=\epsilon \Delta\left(x_{0}, x_{c}\right)=\epsilon \Delta\left(x_{0}, \alpha_{x_{0} x_{1}}(c)\right)=\epsilon c \Delta\left(x_{0}, x_{1}\right)=\Delta\left(x_{0}, \alpha_{x_{0} x_{1}}(\epsilon c)\right)
$$

leading then to $\alpha_{x_{0} x_{c}}(\epsilon)=\alpha_{x_{0} x_{1}}(\epsilon c)$.

\section{Reduction and discretization of variational principles on Lie groups}

We present here the discretization and numerical integration mechanisms introduced for Euler-Poincaré equations in mechanics $[5,8,27,28,29,39]$. For a given Lie group $G$, assume we have a Lagrangian density $\mathcal{L}(t, g, \dot{g}) \mathrm{d} t$ determined by a smooth Lagrangian function $\mathcal{L}: \mathbb{R}_{t} \times T G \rightarrow \mathbb{R}$, and the corresponding action functionals $\mathbb{L}_{K}(g(t))=\int_{K} \mathcal{L}(t, g(t), \dot{g}(t)) \mathrm{d} t$ defined for compact intervals $K \subset \mathbb{R}_{t}$ (so that, integration makes sense) and smooth trajectories $g: t \in \mathbb{R} \mapsto g(t) \in G$. Critical trajectories for these action functionals and for infinitesimal variations, whose support is in the interior of $K$, are characterized by Euler-Lagrange equations of the Lagrangian density:

$$
0=\left(\mathrm{d}_{e} l_{g(t)}\right)^{*}\left(\frac{\partial \mathcal{L}}{\partial g}(t, g(t), \dot{g}(t))-\frac{\mathrm{d}}{\mathrm{d} t}\left(\frac{\partial \mathcal{L}}{\partial \dot{g}}(t, g(t), \dot{g}(t))\right)\right) \in \operatorname{Map}\left(\mathbb{R},(\operatorname{Lie} G)^{*}\right)
$$

where $l_{g}: G \rightarrow G$ is the left-product by an element $g \in G$ and the usual Euler-Lagrange equations have been translated to $(\operatorname{Lie} G)^{*}=\left(T_{e} G\right)^{*}$ using $\left(\mathrm{d}_{e} l_{g}\right)^{*}: \theta \in\left(T_{g} G\right)^{*} \mapsto \theta \circ \mathrm{d}_{e} l_{g} \in(\operatorname{Lie} G)^{*}$, in order to have an expression on a common space $(\operatorname{Lie} G)^{*}$, instead of having it on different spaces $\left(T_{g(t)} G\right)^{*}$, for all $t \in \mathbb{R}$. In the case that the Lagrangian is invariant for the action on the left of the Lie group on itself (that is, $\mathcal{L}(t, g, \dot{g})=\mathcal{L}(t, h g, h \dot{g})$ ) this density can be written as $\ell\left(t, g^{-1} \dot{g}\right) \mathrm{d} t$, where the smooth function $\ell(t, \xi): \mathbb{R}_{t} \times \operatorname{Lie} G \rightarrow \mathbb{R}$ is the reduced Lagrangian. Euler-Lagrange equations take the Euler-Poincaré form [34]:

$$
0=\operatorname{ad}_{\xi(t)}^{*} \frac{\partial \ell}{\partial \xi}(t, \xi(t))-\frac{\mathrm{d}}{\mathrm{d} t}\left(\frac{\partial \ell}{\partial \xi}(t, \xi(t))\right) \in \operatorname{Map}\left(\mathbb{R},(\operatorname{Lie} G)^{*}\right), \quad \xi(t)=g(t)^{-1} \dot{g}(t) \in \operatorname{Map}(\mathbb{R}, \operatorname{Lie} G)
$$

where $\operatorname{ad}_{\xi}^{*}$ is the linear endomorphism on $(\operatorname{Lie} G)^{*}$ induced by the adjoint representation of Lie algebras ad: Lie $G \rightarrow \operatorname{End}(\operatorname{Lie} G)$. Determining trajectories $g(t)$ that satisfy Euler-Lagrange equations is reduced to 
finding solutions $\xi(t): \mathbb{R} \rightarrow \operatorname{Lie} G$ of Euler-Poincaré equations and then solving the reconstruction equation $\xi(t)=(g(t))^{-1} \dot{g}(t)$.

Fixing a (reduced/covariant) retraction map on $G$ and a discrete sequence of time events allows to discretize this situation. If we fix an unbounded increasing sequence of time points $\left(t_{k}\right)_{k \in \mathbb{Z}}$ (hence with a finite number of terms on any compact interval), with time-steps $h_{k}=t_{k+1}-t_{k}>0$ (usually constant time-steps), we may seek for a discrete sequence of configurations $\left(g_{k}\right)_{k \in \mathbb{Z}}$, that serve as good approximation of the values $g\left(t_{k}\right)$ of a smooth trajectory on our time grid. We also need values that serve as good approximations of $\xi\left(t_{k}\right)$, for the curve $\xi(t)=g(t)^{-1} \dot{g}(t)$. This is achieved using the following result:

Lemma 3.1

Consider a smooth trajectory $g(t) \in \operatorname{Map}(\mathbb{R}, G)$ on a Lie group $G$ and the induced trajectory $\xi(t)=g(t)^{-1} \dot{g}(t) \in$ $\operatorname{Map}(\mathbb{R}, \operatorname{Lie} G)$ on its Lie algebra. Consider any reduced retraction map $\tau: U_{0} \subset$ Lie $G \rightarrow G$ and the Lie algebra elements:

$$
\xi_{0}(\epsilon)=\frac{\tau^{-1}\left(g_{0}^{-1} g_{1}(\epsilon)\right)}{t_{1}(\epsilon)-t_{0}} \in \operatorname{Lie} G
$$

associated to points $\left(t_{0}, g_{0}=g\left(t_{0}\right)\right),\left(t_{1}(\epsilon)=t_{0}+\epsilon, g_{1}(\epsilon)=g\left(t_{0}+\epsilon\right)\right)$ on the trajectory graphic $\{(t, g(t))\}_{t \in \mathbb{R}}$, for two temporal events $t_{0}$, and $t_{1}(\epsilon)$ with $\epsilon>0$. There exists $\epsilon_{\max }$ such that, for $0<\epsilon<\epsilon_{\max }$, the elements $\xi_{0}(\epsilon)$ are well defined and there holds:

$$
\lim _{\epsilon \rightarrow 0^{+}} \xi_{0}(\epsilon)=\xi\left(t_{0}\right)
$$

\section{Proof}

As $\lim _{\epsilon \rightarrow 0} g\left(t_{0}+\epsilon\right)=g\left(t_{0}\right)=g_{0}$, we have $\lim _{\epsilon \rightarrow 0} g_{0}^{-1} g_{1}(\epsilon)=e$ and as we know that $\tau\left(U_{0}\right)$ is an open neighbourhood of $e \in G$, we may conclude the existence of $\epsilon_{\max }$ such that $g_{0}^{-1} g_{1}(\epsilon) \in \tau\left(U_{0}\right)$, when $0<\epsilon<\epsilon_{\max }$. Applying $\tau^{-1}$ to this element and dividing by $t_{1}(\epsilon)-t_{0}$, which is possible if $t_{1}(\epsilon) \neq t_{0}$ (that is, for $\epsilon \neq 0$ ).

As $g_{1}(0)=g_{0}$ and $\tau^{-1}(e)=0$ we get:

$$
\xi_{0}(\epsilon)=\frac{\tau^{-1}\left(g_{0}^{-1} g_{1}(\epsilon)\right)}{t_{1}(\epsilon)-t_{0}}=\frac{\tau^{-1}\left(g_{0}^{-1} g_{1}(\epsilon)\right)-\tau^{-1}\left(g_{0}^{-1} g_{1}(0)\right)}{\epsilon}
$$

Therefore, taking the limit as $\epsilon \rightarrow 0$ leads to the definition of tangent vector at $\epsilon=0$, for a trajectory $\tau^{-1}\left(g_{0}^{-1} g_{1}(\epsilon)\right)$ on the vector space Lie $G$. Applying the chain rule and knowing $(d / d \epsilon)_{0} g_{1}(\epsilon)=(d / d \epsilon)_{0} g\left(t_{0}+\epsilon\right)=\dot{g}\left(t_{0}\right)$, we get

$$
\lim _{\epsilon \rightarrow 0} \xi_{0}(\epsilon)=\left(\mathrm{d}_{0} \tau\right)^{-1}\left(g_{0}^{-1} \dot{g}\left(t_{0}\right)\right)
$$

which, using $g_{0}=g\left(t_{0}\right), \mathrm{d}_{0} \tau=\mathrm{Id}$ and the definition $\xi(t)=g(t)^{-1} \dot{g}(t)$, proves our statement.

As a consequence, if $g_{0}, g_{1}$ are considered to be good approximations of $g\left(t_{0}\right), g\left(t_{1}\right)$, for two time events with small time-step $t_{1}-t_{0}=h_{0}>0$, the element $\xi_{0}=\frac{\tau^{-1}\left(g_{0}^{-1} g_{1}\right)}{h_{0}}$ should be considered a good approximation to $\xi\left(t_{0}\right)$. The application of the rectangle quadrature rule to approximate the integral $\int_{t_{0}}^{t_{1}} \mathcal{L}(t, g(t), \dot{g}(t)) \mathrm{d} t$, for the function $\mathcal{L}(t, g, \dot{g})=\ell\left(t, g^{-1} \dot{g}\right)$, on a compact interval $I_{0}=\left[t_{0}, t_{1}\right]$, leads to the approximate value

$$
\int_{t_{0}}^{t_{1}} \mathcal{L}(t, g(t), \dot{g}(t)) \mathrm{d} t \simeq \mathcal{L}\left(t_{0}, g\left(t_{0}\right), \dot{g}\left(t_{0}\right)\right)\left(t_{1}-t_{0}\right)=\ell\left(t_{0}, g\left(t_{0}\right)^{-1} \dot{g}\left(t_{0}\right)\right) h_{0} \simeq \ell\left(t_{0}, \xi_{0}\right) h_{0}
$$

\section{Definition 3.1}

We call discrete Lagrangian induced by the reduced Lagrangian density $\ell(t, \xi) \mathrm{d} t$ and the reduced retraction map $\tau: U_{0} \rightarrow G$, the following function defined on an open subset of $(\mathbb{R} \times G) \times(\mathbb{R} \times G)$ :

$$
\mathcal{L}_{d}\left(t_{0}, g_{0}, t_{1}, g_{1}\right)=\ell\left(t_{0}, \xi_{0}\right) h_{0}, \quad \xi_{0}=\frac{\tau^{-1}\left(g_{0}^{-1} g_{1}\right)}{t_{1}-t_{0}}, h_{0}=t_{1}-t_{0}
$$


We know that $\tau^{-1}$ is defined on some neighborhood of $e \in G$ and the quotient is possible when $t_{1} \neq t_{0}$. Therefore $\mathcal{L}_{d}$ is well defined and smooth on an open subset $\widetilde{\mathbb{R} \times G} \subset(\mathbb{R} \times G) \times(\mathbb{R} \times G)$ not including diagonal points, but adherent to these diagonal points.

Recall that for small time-step $t_{k+1}-t_{k}>0$, the value $\mathcal{L}_{d}\left(t_{k}, g_{k}, t_{k+1}, g_{k+1}\right)$ with $g_{k}=g\left(t_{k}\right), g_{k+1}=g\left(t_{k+1}\right)$ can be considered as a good approximation for $\int_{t_{k}}^{t_{k+1}} \ell\left(t, g(t)^{-1} \dot{g}(t)\right) \mathrm{d} t$. Then, the smooth action functional $\mathbb{L}_{K}(g(t))=\int_{K} \mathcal{L}(t, g(t), \dot{g}(t)) \mathrm{d} t$ associated to the Lagrangian density $\ell\left(t, g^{-1} \dot{g}\right) \mathrm{d} t$ on a compact set $K \subset \mathbb{R}$ can be approximated as:

$$
\mathbb{L}_{K}(g(t)) \simeq \mathbb{L}_{K}\left(g_{i}\right)_{i \in \mathbb{Z}}=\sum_{\left[t_{k}, t_{k+1}\right] \subset K} \ell\left(t_{k}, \xi_{k}\right) h_{k}, \quad \xi_{k}=\frac{\tau^{-1}\left(g_{k}^{-1} g_{k+1}\right)}{h_{k}}, \quad h_{k}=t_{k+1}-t_{k}
$$

Here, the definition of $\xi_{k}$ leads to

$$
g_{k+1}=g_{k} \tau\left(h_{k} \xi_{k}\right)
$$

Let us now determine critical points $\left(g_{i}\right)_{i \in \mathbb{Z}} \in \operatorname{Map}(\mathbb{Z}, G)$ of this discrete action functional. For any fixed $k \in \mathbb{Z}$, when we consider a compact interval $K$ large enough so that $t_{k-1}, t_{k}, t_{k+1} \in K$, demanding that a sequence of configurations $\left(g_{i}\right)_{i \in \mathbb{Z}}$ is critical for the discrete action functional $\mathbb{L}_{K}$ leads to the condition that, for each $\xi \in \operatorname{Lie} G$, the action functional restricted to the curve $g_{k}(\epsilon)=g_{k} \exp \epsilon \xi, g_{i}=$ constant $(\forall i \neq k)$ is a function $\mathbb{L}_{K}(\epsilon)$ with a stationary value at $\epsilon=0$. As we are varying a single component $g_{k}$, the summation defining $\mathbb{L}_{K}(\epsilon)$ has many constant terms, being the only non-constant ones precisely those that explicitly depend on $g_{k}$. This dependence may arise only in the terms $\xi_{k-1}, \xi_{k} \in \operatorname{Lie} G$. Stationarity at $\epsilon=0$ implies then:

$$
\begin{aligned}
0= & \left(\frac{d}{d \epsilon}\right)_{\epsilon=0}\left(\ell\left(t_{k-1}, \xi_{k-1}(\epsilon)\right) h_{k-1}+\ell\left(t_{k}, \xi_{k}(\epsilon)\right) h_{k}\right) \\
& \text { where } \tau\left(h_{k} \xi_{k}(\epsilon)\right)=\left(g_{k} \exp \epsilon \xi\right)^{-1} g_{k+1}=(\exp -\epsilon \xi) g_{k}^{-1} g_{k+1}=r_{g_{k}^{-1} g_{k+1}} \exp (-\epsilon \xi) \\
& \tau\left(h_{k-1} \xi_{k-1}(\epsilon)\right)=g_{k-1}^{-1} g_{k} \exp \epsilon \xi=r_{g_{k-1}^{-1} g_{k}} \exp \epsilon \operatorname{Ad}_{g_{k-1}^{-1} g_{k}} \xi
\end{aligned}
$$

Here, $r_{g}: G \rightarrow G$ stands for the right product with an element $g \in G$ and Ad: $G \rightarrow \operatorname{Aut}(\operatorname{Lie} G)$ stands for the adjoint representation of the Lie group on its Lie algebra (hence $\exp \operatorname{Ad}_{h} \xi=r_{h}^{-1} \circ l_{h}(\exp \xi)$ ).

The differential of $\tau$ at any given point $\xi \in \operatorname{Lie} G$ can be described as $\mathrm{d}_{\xi} \tau: T_{\xi} \operatorname{Lie} G \rightarrow T_{\tau(\xi)} G$ or, using natural identifications $T_{\xi} \operatorname{Lie} G \simeq \operatorname{Lie} G$ and $\left(\mathrm{d}_{e} r_{g}\right)^{-1}: T_{g} G \simeq T_{e} G=\operatorname{Lie} G$, can be described as an endomorphism $(\mathrm{d} \tau)_{\xi}=\left(\mathrm{d}_{e} r_{\tau(\xi)}\right)^{-1} \circ \mathrm{d}_{\xi} \tau \in \operatorname{End}(\operatorname{Lie} G)$ (the trivialized differential). In this situation, deriving the relations above:

$$
\begin{aligned}
& h_{k}\left(\frac{d}{d \epsilon}\right)_{\epsilon=0} \xi_{k}(\epsilon)=-(\mathrm{d} \tau)_{h_{k} \xi_{k}}^{-1} \xi, \\
& h_{k-1}\left(\frac{d}{d \epsilon}\right)_{\epsilon=0} \xi_{k-1}(\epsilon)=(\mathrm{d} \tau)_{h_{k-1} \xi_{k-1}}^{-1} \operatorname{Ad}_{\tau\left(h_{k-1} \xi_{k-1}\right)} \xi
\end{aligned}
$$

therefore, taking the derivative at $\epsilon=0$ we get, as a necessary condition for criticality, the discrete Euler-Poincaré equations [33]:

$$
0=\left(\frac{\partial \ell}{\partial \xi}\left(t_{k-1}, \xi_{k-1}\right)\right) \circ(\mathrm{d} \tau)_{h_{k-1} \xi_{k-1}}^{-1} \circ \operatorname{Ad}_{\tau\left(h_{k-1} \xi_{k-1}\right)}-\left(\frac{\partial \ell}{\partial \xi}\left(t_{k}, \xi_{k}\right)\right) \circ(\mathrm{d} \tau)_{h_{k} \xi_{k}}^{-1}
$$

where $\frac{\partial \ell}{\partial \xi} \in(\operatorname{Lie} G)^{*}$ stands for the Lie $G$-component of the differential of the function $\ell: \mathbb{R} \times$ Lie $G \rightarrow \mathbb{R}$, at any point $(t, \xi)$, and $(\mathrm{d} \tau)_{\xi}$ is the endomorphism on Lie $G$ determined by the differential of $\tau$, at any point $\xi \in \operatorname{Lie} G$, using the right-trivialization to identify $T_{g} G$ with Lie $G$, as indicated above.

Equivalently, we may state:

$$
\mu_{k}=\operatorname{Ad}_{\tau\left(h_{k-1} \xi_{k-1}\right)}^{*} \mu_{k-1}
$$


where $\operatorname{Ad}_{g}^{*} \in \operatorname{Aut}\left((\operatorname{Lie} G)^{*}\right)$ is the linear automorphism on $(\operatorname{Lie} G)^{*}$ induced by the adjoint representation Ad: $G \rightarrow \operatorname{Aut}(\operatorname{Lie} G)$ of $G$ on its Lie algebra, and where we define

$$
\mu_{k}=\left(\frac{\partial \ell}{\partial \xi}\left(t_{k}, \xi_{k}\right)\right) \circ(\mathrm{d} \tau)_{h_{k} \xi_{k}}^{-1}=\mu\left(k, \xi_{k}\right)
$$

In this regard the mapping

$$
\operatorname{Mom}_{\tau, \ell}:(k, \xi) \in \mathbb{Z} \times \operatorname{Lie} G \mapsto(k, \mu(k, \xi)) \in \mathbb{Z} \times(\operatorname{Lie} G)^{*}, \quad \mu(k, \xi)=\left(\frac{\partial \ell}{\partial \xi}\left(t_{k}, \xi\right)\right) \circ(\mathrm{d} \tau)_{h_{k} \xi}^{-1}
$$

plays a central role. It shall be called the momentum map associated to the reduced Lagrangian $\ell$ and retraction map $\tau$. Values $\mu_{k}=\mu\left(k, \xi_{k}\right)$ are called Lie $G$-valued momenta at time $t_{k}$.

Equations (2), (4) (definition of discrete trivialized velocity $\xi_{k}$ and momentum $\mu_{k}$, respectively), together with equation (3) (condition of criticality for the discrete action functional) represent the three steps that allow a discrete integration of Euler-Poincaré equations, using an iterative scheme. The hard part of the integration scheme is reduced to the determination of a right-inverse momentum map

$$
M_{\tau o m}^{-1}:(k, \theta) \in \mathbb{Z} \times(\operatorname{Lie} G)^{*} \mapsto(k, \xi(k, \theta)) \in \mathbb{Z} \times \operatorname{Lie} G
$$

characterized by $M o m_{\tau, \ell} \circ M o m_{\tau, \ell}^{-1}=\mathrm{Id}$, that is, $\mu(k, \xi(k, \theta))=\theta, \forall k \in \mathbb{Z}, \forall \theta \in(\operatorname{Lie} G)^{*}$. Integrating discrete Euler-Poincaré equations relies in:

- From $\mu_{i-1}$ and using $\operatorname{Mom}_{\tau, \ell}^{-1}(k, \theta)=(k, \xi(k, \theta))$ one may obtain $\xi_{i-1}=\xi\left(i-1, \mu_{i-1}\right)$, for which (4) holds (at the $k=i-1$ value). This inverse could be replaced by some algorithm that determines numerical solutions of the equation $\operatorname{Mom}_{\tau, \ell}\left(i-1, \xi_{i-1}\right)=\left(i-1, \mu_{i-1}\right)$.

- From $\xi_{i-1}, \mu_{i-1}$ and using $\tau$ one may obtain $\mu_{i}$, for which (3) holds (at the $k=i$ value).

Iterative application of the first two steps allows to recover $\left(\mu_{k}, \xi_{k}\right)_{k \in \mathbb{N}}$ from $\mu_{0}$.

- From $\left(g_{i-1}, \xi_{i-1}\right)$, and using $\tau$ one may obtain $g_{i}$ for which (2) holds (at the $k=i-1$ value).

Finally application of the last step allows to recover $\left(g_{k}, \mu_{k}, \xi_{k}\right)_{k \in \mathbb{N}}$ from $\left(g_{0}, g_{1}\right)$, from $\left(g_{0}, \xi_{0}\right)$ or from $\left(g_{0}, \mu_{0}\right)$ (which are related one to another by (2) and by (4) at $k=0$ ). Observe that in this iterative scheme the original smooth reduced Lagrangian $\ell$ is used and the specific computation of $\tau^{-1}$ or its derivative is not needed, except possibly for the computation of $\xi_{0}$ if we give $\left(g_{0}, g_{1}\right)$ as initial data.

The reduction procedure we have presented, translating the determination of a trajectory $g(t)$ on the Lie group into a new problem of determining a trajectory $\xi(t)$ on the Lie algebra is not specific of geometric mechanics. It also appears in the dynamics of continuous media and in field theories. For field theories on a principal $G$-bundle that admit the structure group $G$ as symmetries, the corresponding variational formalism leads to Euler-Poincaré equations, as described in $[14,15]$. In the case of dynamics of continuous media, the corresponding field theories have been discretized $[18,25]$, using a very large group $G$, where each single element contains all configurations of several points in the media, choosing then a particular retraction map on this large group, and representing the

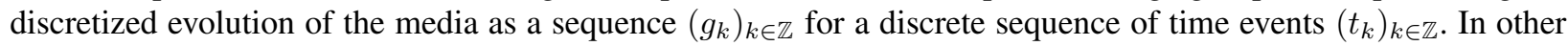
situations, Euler-Poincaré equations in field theories have been discretized [13, 19, 39], using fields on $\mathbb{Z}^{2}$ with values on a simple Lie group, that is, considering fields as sections of the trivial bundle $\mathbb{Z}^{2} \times G \rightarrow \mathbb{Z}^{2}$. In the same particular case, [39] explored the reduction process using the notion of discrete connection in this particular situation.

\section{Reduction of a Lagrangian density in field theories}

Before discretizing and reducing by some group of symmetries the main elements of variational field theories, we present in a suitable form the Ehresmann theory of jets and connections on fibered manifolds. 


\subsection{The connection and jet bundles}

A field theory deals with local sections of a bundle $\pi: Y \rightarrow X$. Such sections are also called (local) fields. For any bundle $\pi: Y \rightarrow X$ the linear morphism $\mathrm{d} \pi: T Y \rightarrow \pi^{*} T X$ is surjective and determines an exact sequence of vector bundles over $Y$ :

$$
0 \rightarrow V Y \hookrightarrow T Y \rightarrow \pi^{*} T X \rightarrow 0
$$

\section{Definition 4.1}

Splittings of the exact sequence (5) are called connections on the bundle $\pi: Y \rightarrow X$.

Any splitting can be determined as a linear immersion $\pi^{*} T X \rightarrow T Y$ covering $\operatorname{Id}_{T X}$. It is equivalent to give the immersion or to give its image (we know that the morphism covers $\operatorname{Id}_{T X}$ ), a vector sub-bundle Hor $\subset T Y$ transversal to the projection $\mathrm{d} \pi$, that is, such that the restricted projector $\left.\mathrm{d} \pi\right|_{\mathrm{Hor}}$ : Hor $\rightarrow \pi^{*} T X$ is an isomorphism. Elements in Hor are called horizontal tangent vectors on $Y$ with respect to the connection.

Observe that a linear mapping $J_{y}: T_{x} X \rightarrow T_{y} Y$ (with $x=\pi(y)$ ) covers the identity on $T_{x} X$ if and only if $\mathrm{d}_{y} \pi \circ J_{y}=\operatorname{Id}_{x}: T_{x} X \rightarrow T_{x} X$. Recall that linear morphisms of vector bundles from $E \rightarrow X$ to $F \rightarrow X$ can be seen as sections of the tensor product bundle $F \otimes E^{*} \rightarrow X$, and the composition $g \circ f \in \Gamma\left(V \otimes E^{*}\right)$ of $f \in \Gamma\left(F \otimes E^{*}\right)$ with $g \in \Gamma\left(V \otimes F^{*}\right)$ gets identified with $c_{F}(g \otimes f)$, where the linear mapping $c_{F}: V \otimes F^{*} \otimes F \otimes E^{*} \rightarrow V \otimes E^{*}$ stands for the contraction on the $F^{*}, F$-components.

\section{Proposition 4.1}

Consider the vector bundle morphism $c_{\pi}: J_{y} \in T Y \otimes \pi^{*} T^{*} X \mapsto c_{T Y}\left(\mathrm{~d}_{y} \pi \otimes J_{y}\right) \in \pi^{*}\left(T X \otimes T^{*} X\right)$. There is a one-to-one correspondence between connections (splittings of the exact sequence (5)) and global sections of the bundle $T Y \otimes \pi^{*} T^{*} X$ for which $c_{\pi}\left(J_{y}\right)=\operatorname{Id}_{\pi(y)} \in\left(\pi^{*} T X \otimes \pi^{*} T^{*} X\right)_{y}$ holds.

As $c_{\pi}$ is a morphism of vector bundles and $\operatorname{Id}_{T X}: Y \rightarrow \pi^{*} T X \otimes \pi^{*} T^{*} X$ is a global section, the equation $c_{\pi}\left(J_{y}\right)=\operatorname{Id}_{\pi(y)}$ determines an affine sub-bundle $J Y \subseteq T Y \otimes \pi^{*} T^{*} X$, modelled over the vector sub-bundle $\operatorname{ker} c_{\pi}=V Y \otimes \pi^{*} T^{*} X \subseteq T Y \otimes \pi^{*} T^{*} X$.

\section{Definition 4.2}

We call bundle of connections associated to $Y \rightarrow X$ and denote it by $\pi_{J}: J Y \rightarrow Y$, the sub-bundle of elements $J_{y} \in T_{y} Y \otimes T_{\pi(y)}^{*} X$ for which $c_{\pi}\left(J_{y}\right)=\operatorname{Id}_{\pi(y)}$ holds.

We call bundle of first-jets of sections associated to $Y \rightarrow X$ (or simply jet-bundle associated to $Y$ ) the bundle $j \pi: J Y \rightarrow X\left(\right.$ where $\left.j \pi=\pi \circ \pi_{J}\right)$.

As a manifold, the bundle of jets and the bundle of connections coincide, but the fibration is considered on different base manifolds. Global sections $J \in \Gamma(Y, J Y)$ of the bundle of connections can be seen as connections on the bundle $Y \rightarrow X$, that is, as splittings of the exact sequence (5). Local sections of the bundle of connections shall be called local connections on the bundle $Y \rightarrow X$.

Each local section $y: X \rightarrow Y$ determines at each point on its domain a linear morphism $\mathrm{d}_{x} y: T_{x} X \rightarrow$ $T_{y(x)} Y$ (which we might represent by the Jacobian matrix associated to the mapping $y$ ). This allows to define a local section $j y: X \rightarrow T Y \otimes \pi^{*} T^{*} X$ of the vector bundle $T Y \otimes \pi^{*} T^{*} X$. This section takes values on the affine sub-bundle $J Y \subseteq T Y \otimes \pi^{*} T^{*} X$ and $j y \in \Gamma(j \pi)$ is called the jet extension of $y \in \Gamma(Y)$.

A system of first order partial differential equations is simply a submanifold $\mathcal{D} \subset J Y$. Solutions of such a system are local sections $y \in \Gamma(Y)$ whose jet extension $j y \in \Gamma(j \pi)$ take values on the submanifold $\mathcal{D}$. A section $\sigma \in \Gamma(j \pi)$ is a holonomic section of the jet bundle if it is a jet extension of some section $y \in \Gamma(Y)$, necessarily the jet extension of the projected section $y=\pi_{J} \circ \sigma: X \rightarrow Y$. Holonomic sections $\sigma$ are characterized by the condition $\sigma=j\left(\pi_{J} \circ \sigma\right)$, which represent a system of first order partial differential equations for sections of $j \pi: J Y \rightarrow X$ (the holonomy system of equations for sections on $J Y$ ). Seeking for a solution of a system of differential equations $\mathcal{D}$ is equivalent to seeking local sections $\sigma \in \Gamma(j \pi)$ that are contained in $\mathcal{D}$ and are holonomic. Higher order partial differential equations might be defined in a similar way [36], using submanifolds of $J(J \ldots(J Y))$. 


\subsection{The bundle of principal connections}

We shall be interested in the behaviour of first order partial differential equations in the presence of a group of symmetries. By this we mean a representation of some Lie group $G$ as automorphisms of the configuration bundle $\pi: Y \rightarrow X$ of the field theory.

\section{Definition 4.3}

An action of a Lie group $G$ on a bundle $\pi: Y \rightarrow X$ is a morphism of groups $\lambda: g \in G \mapsto \lambda_{g} \in \operatorname{Aut}(Y)$ from $G$ to the group of bundle automorphisms. We say the action is smooth if $a c:(g, y) \in G \times Y \mapsto \lambda_{g}(y) \in Y$ is smooth (in a similar way, we may talk of proper actions if $a c$ is proper). Each $\lambda_{g}: Y \rightarrow Y$ projects into $\lambda_{g}^{X}: X \rightarrow X$, defining thus an action on the base manifold $X$. We call fibered action any smooth action such that $\lambda_{g}^{X}=\operatorname{Id}_{X}$ (hence the group morphism $\lambda$ takes values on the subgroup $\operatorname{Aut}_{X}(Y)$ of $X$-bundle automorphisms).

The most simple situation of a fibered group action appears when we have a smooth proper action of a Lie group $G$ on the bundle $\pi: Y \rightarrow X$, by $X$-bundle morphisms and free and transitive on each fiber (so that $X=Y / G$ ). This is the case of a principal $G$-bundle.

\section{Definition 4.4}

For any Lie group $G$ we call principal $G$-bundle any fiber bundle $\pi: P \rightarrow X$, together with a proper, smooth, fibered action of $G$ on $P$, that is free and transitive on the fibers of $P$.

The automorphism $\lambda_{g}: P_{x} \rightarrow P_{x}$ is called the left translation determined by $g \in G$ on the fiber $P_{x}$. Using the notation $\lambda_{g}\left(p_{x}\right)=g p_{x}$ for left translations there holds:

$$
\pi\left(g p_{x}\right)=\pi\left(p_{x}\right)=x, \quad g\left(h p_{x}\right)=(g h) p_{x}, \quad G p_{x}=P_{x}, \quad g p_{x}=p_{x} \Leftrightarrow g=e
$$

\section{Remark 4.1}

For historical reasons, and originating in Ehresmann [21], principal $G$-bundles are usually defined using a group anti-homomorphism from $G$ to $\operatorname{Aut}_{X}(P)$, that is, using right actions instead of left actions. However, in geometric mechanics the principal bundle $\mathbb{R} \times G \rightarrow \mathbb{R}$ is implicit in each step, and the reduction mechanism described in section 3 is always performed for the left action of $G$ on this bundle. To maintain the parallelism of reduced variational field theories with reduced mechanical formalisms, we adopt the less common choice of left actions in the definition of a principal bundle.

Apart from the group $G$, faithfully represented as the group of left-translations on any fiber, a second subgroup of automorphisms is relevant in the theory of principal bundles: the subgroup of gauge transformations on a fiber.

\section{Definition 4.5}

We denote by Gau $P_{x}$ and call Gauge group associated to a fiber $P_{x}$ of a principal $G$-bundle $\pi: P \rightarrow X$, the group of automorphisms of the fiber $P_{x}$ that commute with all left-translations $\lambda_{g}$.

The set of all gauge transformations Gau $P=\sqcup_{x \in X}$ Gau $P_{x}$ has a natural smooth bundle structure $\pi_{\text {Gau }}$ : Gau $P \rightarrow X$, called gauge bundle, where each fiber Gau $P_{x}$ is a Lie group, with composition of mappings as product and the identity mapping $\operatorname{Id}_{x}: P_{x} \rightarrow P_{x}$ as unit element.

\section{Definition 4.6}

For a Lie group $(G, \cdot)$, we call reverse Lie group structure the one induced in the manifold $G$ by the reversed product $\circ$, defined as $g \circ h=h \cdot g$. The manifold $G$ with the reverse product $\circ$ is a different Lie group that shall be denoted by $G^{r}$.

Principal $G$-bundles defined as a group anti-homomorphism $\rho: G \rightarrow \operatorname{Aut}_{X}(P)\left(\right.$ right action $\rho_{g h}=\rho_{h} \circ \rho_{g}$ ) can be seen as principal $G^{r}$-bundles (in this case $\rho$ is a left-action, because $\rho_{h \circ g}=\rho_{h} \circ \rho_{g}$ for the element $h \circ g=g h$ ).

The following notation recovers Ehresmann's original notation [21], with a reversed writing due to our choice of left actions in the definition of a principal $G$-bundle:

Definition 4.7

Let $\pi: P \rightarrow X$ be a principal $G$-bundle. For any pair $\left(p_{x}, \bar{p}_{x}\right) \in P \times_{X} P$ we denote by $\bar{p}_{x} p_{x}^{-1} \in G$ the unique 
group element $g \in G$ whose associated left-translation transforms $p_{x}$ into $\bar{p}_{x}$ (that is $g p_{x}=\bar{p}_{x}$ ). The mapping $\left(p_{x}, \bar{p}_{x}\right) \in P \times{ }_{X} P \mapsto \bar{p}_{x} p_{x}^{-1} \in G$ shall be called the group difference map on the principal $G$-bundle $P$.

For any pair $\left(p_{x}, \bar{p}_{x}\right) \in P \times_{X} P$ the mapping $q_{x} \in P_{x} \mapsto\left(q_{x} p_{x}^{-1}\right) \bar{p}_{x} \in P_{x}$ is a gauge transformation on the fiber $P_{x}$, the only one transforming $p_{x}$ into $\bar{p}_{x}$. We denote this transformation as $p_{x}^{-1} \bar{p}_{x} \in$ Gau $P_{x}$. The mapping $\left(p_{x}, \bar{p}_{x}\right) \in P \times_{X} P \mapsto p_{x}^{-1} \bar{p}_{x} \in$ Gau $P$ shall be called the gauge difference map on the principal $G$-bundle $P$.

Ehresmann (with a reversed convention in [21]) characterized as $P_{x}^{-1} P_{x}$ the group $G$ of left translations and $P_{x} P_{x}^{-1}$ the gauge group Gau $P_{x}$ on any fiber of the principal $G$-bundle. On the Lie group (Gau $P_{x}, \circ$ ), we may denote the reverse product by - or simply by juxtaposition of elements, therefore for $\varphi_{x}, \phi_{x} \in$ Gau $P_{x}$ we denote by $\phi_{x} \varphi_{x}=\phi_{x} \cdot \varphi_{x}=\varphi_{x} \circ \phi_{x} \in$ Gau $P_{x}$ the automorphism $p_{x} \in P_{x} \mapsto \varphi_{x}\left(\phi_{x}\left(p_{x}\right)\right) \in P_{x}$. Using these notations and $\phi_{x}\left(p_{x}\right)=p_{x} \phi_{x}$, for gauge transformations acting on a fiber $P_{x}$, we have $\left(\forall \phi_{x}, \varphi_{x} \in\right.$ Gau $P_{x}, \forall p_{x}, q_{x}, r_{x} \in P_{x}$ $\forall g \in G)$

$$
\begin{array}{lrl}
g\left(p_{x} \phi_{x}\right)=\left(g p_{x}\right) \phi_{x}, & \left(q_{x} p_{x}^{-1}\right) p_{x}=q_{x}, & q_{x}\left(q_{x}^{-1} p_{x}\right)=p_{x} \\
\pi\left(p_{x} \phi_{x}\right)=\pi\left(p_{x}\right)=x, & \left(p_{x} \phi_{x}\right) \varphi_{x}=p_{x}\left(\phi_{x} \varphi_{x}\right), & p_{x} \phi_{x}=p_{x} \Leftrightarrow \phi_{x}=\operatorname{Id}_{x} \\
\left(g q_{x}\right) p_{x}^{-1}=g\left(q_{x} p_{x}^{-1}\right), & p_{x}^{-1}\left(q_{x} \phi_{x}\right)=\left(p_{x}^{-1} q_{x}\right) \phi_{x}, & p_{x}\left(q_{x}^{-1} r_{x}\right)=\left(p_{x} q_{x}^{-1}\right) r_{x} \\
p_{x} q_{x}^{-1}=e \Leftrightarrow p_{x}=q_{x}, & p_{x} q_{x}^{-1}=\left(q_{x} p_{x}^{-1}\right)^{-1}, & \left(p_{x} \phi_{x}\right)\left(q_{x} \phi_{x}\right)^{-1}=p_{x} q_{x}^{-1} \\
p_{x}^{-1} q_{x}=\mathrm{Id}_{x} \Leftrightarrow p_{x}=q_{x} & q_{x}^{-1} p_{x}=\left(p_{x}^{-1} q_{x}\right)^{-1}, & \left(g p_{x}\right)^{-1}\left(g q_{x}\right)=p_{x}^{-1} q_{x}
\end{array}
$$

The listed properties can be proved from the corresponding definitions. Recall that there is no meaning for the concept of a product or the inverse of elements in $P_{x}$ (in the same manner as there is no meaning for the concept of addition or opposite of points in an affine space, but the difference of points is meaningful as an element in the underlying vector space).

\section{Remark 4.2}

On a trivial $G$-bundle $P=X \times G$, there holds $(x, \bar{g})(x, g)^{-1}=\bar{g} g^{-1} \in G$. Moreover, all gauge transformations on a fibe $P_{x}$ of this bundle have the form $r_{h}:(x, g) \mapsto(x, g h)$, the reverse product of any pair of gauge transformations is $r_{h} \cdot r_{\bar{h}}=r_{\bar{h}} \circ r_{h}=r_{h \bar{h}}$, and the only gauge transformation that takes $(x, g)$ into $(x, \bar{g})$ is $r_{h}$ for $h=g^{-1} \bar{g}$. This justifies our notation choice and establishes an identification Gau $P=X \times G^{r}$, for the trivial bundle $P=X \times G$.

Smooth actions of a Lie group $G$ by $\lambda_{g}$ on a bundle $\pi: Y \rightarrow X$ induce smooth actions on $T Y$ by $\mathrm{d} \lambda_{g}$, on $\pi^{*} T^{*} X=Y \times_{X} T^{*} X$ by $\lambda_{g} \times\left(\mathrm{d} \lambda_{g^{-1}}^{X}\right)^{*}$, and on $T Y \otimes \pi^{*} T^{*} X$ by the tensor product of these actions. The submanifold $J Y \subset T Y \otimes \pi^{*} T^{*} X$ is invariant by this action, leading to a new action $j \lambda_{g}$ of the group on $J Y$. This action transforms a jet $j_{x} y$ associated to a section $y(x)$ at $x \in X$ into the jet $j_{\bar{x}} \bar{y}$ associated to the section $\bar{y}=\lambda_{g} \circ y \circ \lambda_{g^{-1}}^{X}$ at $\bar{x}=\lambda_{g}^{X}(x) \in X$. (See [36] for more on group actions on a jet bundle)

We explore now, for the particular case of principal $G$-bundles, the subset of $G$-covariant connections. For each $g \in G$, we have a commutative diagram of linear morphisms, each of them covering $\lambda_{g}: P \rightarrow P$ :

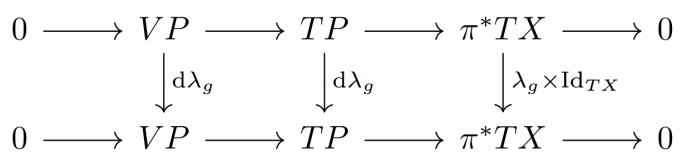

We will not enter into the particular details of the existence of quotient manifolds, when we deal with the structure Lie group and its natural action on natural bundles derived from a principal $G$-bundle (for a good general reference, consult [37]). In all cases considered in our work, the existence of this quotient can be proved taking into account that the principal $G$-bundle is locally trivial and studying the situation for trivial bundles. Taking the quotient by these actions on the exact sequence (5) of vector bundles on $Y=P$, we obtain an induced exact sequence of vector bundles on $X$

$$
0 \rightarrow V P / G \rightarrow T P / G \rightarrow \pi^{*} T X / G \rightarrow 0
$$

\section{Definition 4.8}

We call $\pi_{A t}: T P / G \rightarrow P / G$ Atiyah bundle, and denote it by $\pi_{A t}: T_{G} P \rightarrow X$. We call $\pi_{\mathrm{Ad}}: V P / G \rightarrow P / G$ adjoint bundle, and denote it by $\pi_{\mathrm{Ad}}: \operatorname{Ad} P \rightarrow X$. 
Using these notations we have an exact sequence (so-called Atiyah sequence [3]) of vector bundles:

$$
0 \rightarrow \operatorname{Ad} P \rightarrow T_{G} P \rightarrow T X \rightarrow 0
$$

There is a clear interpretation of the quotients: $P / G=X,\left(\pi^{*} T X\right) / G=T X$. A geometrical interpretation is possible also for the bundles $T_{G} P=T P / G \rightarrow X$ and $\operatorname{Ad} P=V P / G \rightarrow X$.

Consider for any fiber $P_{x} \subset P$, the action mapping $\left(p_{x}, \phi_{x}\right) \in P_{x} \times \operatorname{Gau} P_{x} \mapsto p_{x} \phi_{x} \in P_{x}$. It induces a corresponding linear isomorphism of bundles over $P_{x}$, given by $\left(p_{x}, a_{x}\right) \in P_{x} \times \operatorname{Lie} \operatorname{Gau} P_{x} \mapsto(d / d \epsilon)_{0} p_{x} \exp \epsilon a_{x} \in$ $T\left(P_{x}\right)=(V P)_{x}$ (bijectivity is easy to check working with trivializations $P_{x} \simeq G$, Gau $P_{x} \simeq G^{r}$ of the fiber and gauge group). This linear isomorphism covariates with the action $\lambda_{g} \times \operatorname{Id}$ on $P_{x} \times \operatorname{Lie} \operatorname{Gau} P_{x}$ and $\mathrm{d} \lambda_{g}$ on $V P_{x}$, and leads to a linear isomorphism Lie Gau $P_{x} \rightarrow(V P)_{x} / G=\operatorname{Ad} P_{x}$. To be more specific, for any choice $p_{x} \in P_{x}$ the identification

$$
a_{x} \in \operatorname{Lie} \operatorname{Gau} P_{x} \mapsto \pi^{G}\left((d / d \epsilon)_{0} p_{x} \exp \epsilon a_{x}\right) \in(V P)_{x} / G=\operatorname{Ad} P_{x}
$$

where $\pi^{G}: V P \rightarrow V P / G=\operatorname{Ad} P$, does not depend on the particular choice $p_{x} \in P_{x}$, and establishes a natural isomorphism Lie Gau $P_{x} \simeq \operatorname{Ad} P_{x}$

\section{Proposition 4.2}

The natural mapping $A \in T P \rightarrow\left(\pi_{T P}(A), \pi^{G}(A)\right) \in P \times T_{G} P$ establishes a natural diffeomorphism of the bundle $\pi_{T P}: T P \rightarrow P$ with the pull-back $\pi^{*} T_{G} P=P \times_{\left(\pi, \pi_{A t}\right)} T_{G} P \rightarrow P$ of Atiyah's bundle $\pi_{A t}: T_{G} P \rightarrow X$ by $\pi: P \rightarrow X$. Moreover, the left action $\mathrm{d} \lambda_{g}$ on $T P$ is identified in the pull-back bundle with $\lambda_{g} \times \operatorname{Id}_{T_{G} P}$.

The natural mapping $A \in V P \rightarrow\left(\pi_{V P}(A), \pi^{G}(A)\right) \in P \times \operatorname{Ad} P$ establishes a natural diffeomorphism of the bundle $\pi_{V P}: V P \rightarrow P$ with the pull-back $\pi^{*}$ Ad $P \rightarrow P$ of the adjoint bundle $\pi_{A d}:$ Ad $P \rightarrow X$ by $\pi: P \rightarrow X$. Moreover, the left action $\mathrm{d} \lambda_{g}$ on $V P$ is identified in the pull-back bundle with $\lambda_{g} \times \operatorname{Id}_{\operatorname{Ad} P}$.

$$
T P \simeq \pi^{*} T_{G} P, \quad V P \simeq \pi^{*} \text { Ad } P \text { as bundles with a fibered G-action }
$$

\section{Proof}

We give the proof for Atiyah's bundle (the adjoint bundle case parallels this one). For each $(p, a) \in P \times_{\left(\pi, \pi_{A t}\right)} T_{G} P$ we may consider the fiber $P_{x}$ associated to $x=\pi(p)=\pi_{A t}(a)$. As $G$ acts freely and transitively on this fiber, and $a \in T_{G} P=T P / G$ is an equivalence class, there exists exactly a unique representative $A_{p} \in\left(\pi^{G}\right)^{-1}(a) \subset T P$ with $\pi_{T P}\left(A_{p}\right)=p, \pi^{G}\left(A_{p}\right)=a$. Thus the mapping in the statement is injective and its image is precisely $P \times_{\left(\pi, \pi_{A t}\right)} T_{G} P$. As the given natural mapping is a regular smooth mapping, injectivity allows to conclude that it is a diffeomorphism with its image, which is the pull-back $\pi^{*} T_{G} P=P \times_{\left(\pi, \pi_{A t}\right)} T_{G} P$.

Moreover, for $A_{p} \simeq\left(p, \pi^{G}\left(A_{p}\right)\right) \in T P \simeq P \times_{X} T_{G} P$ the element $\left(\mathrm{d}_{p} \lambda_{g}\right)\left(A_{p}\right) \in T_{g p} P$ gets identified with $\left(g p, \pi^{G} \circ\left(\mathrm{d}_{p} \lambda_{g}\right)\left(A_{p}\right)\right)=\left(g p, \pi^{G}\left(A_{p}\right)\right) \in P \times_{X} T_{G} P$ hence the action $\mathrm{d} \lambda_{g}$ on $T P$ gets identified with $\lambda_{g} \times$ $\operatorname{Id}_{T_{G} P}$.

Following this result, sections $a: x \in X \mapsto a_{x} \in T_{G} P$ extend to sections $A: \pi^{*} X \rightarrow \pi^{*} T_{G} P$, therefore to vector fields $A: P \rightarrow T P$ that are covariant for the action $\lambda_{g}$ on $P$ and $\mathrm{d} \lambda_{g}$ on $T P$, hence elements in $\Gamma\left(X, T_{G} P\right)$ can be seen as vector fields on $P$ that are invariant by the natural action of $G$ on the module of vector fields. Conversely, invariant vector fields $A: P \rightarrow T P$ factor through the action $\lambda_{G}$ on $P$ and $\mathrm{d} \lambda_{g}$ on $T P$ inducing sections $a: P / G=X \rightarrow(T P) / G=T_{G} P$ of the Atiyah bundle. There is an immersion $\Gamma\left(X, T_{G} P\right) \subset \Gamma(P, T P)$, whose image is the set of $G$-invariant vector fields. Atiyah's bundle $T_{G} P$ can be called the bundle of $G$-invariant vector fields on $P$.

The surjective morphism $\mathrm{d} \pi: T P \rightarrow \pi^{*} T X$ induces in the quotient by $G$ a surjective morphism of vector bundles $\overline{\mathrm{d} \pi}: T_{G} P \rightarrow T X$, whose kernel is the subbundle of vertical (over $X$ ) invariant vector fields on $P$. This subbundle may be identified with $V P / G$ (observe that $\mathrm{d} \lambda_{g}$ leaves invariant the vector subbundle $V P \subset T P$, so the quotient makes sense). The adjoint bundle Ad $P$ can be called the bundle of $G$-invariant vertical vector fields on $P$, or bundle of infinitesimal gauge transformations (its fiber are the Lie algebras associated to the gauge Lie groups Gau $P_{x}$ ).

\section{Definition 4.9}

[3] We call principal connection any splitting of the exact sequence (6). 
Again, as in Definition 4.1 such a splitting can be described as a linear immersion $T X \rightarrow T_{G} P$ covering $\operatorname{Id}_{T X}$. It is equivalent to give the immersion or to give its image (we know that the morphism covers $\operatorname{Id}_{T X}$ ), a vector subbundle Hor $\subset T_{G} P$ transversal to the projection $\overline{\mathrm{d} \pi}$, that is, such that the restricted projector $\left.\overline{\mathrm{d} \pi}\right|_{\text {Hor }}:$ Hor $\rightarrow T X$ is an isomorphism.

Similar to Proposition 4.1, we conclude here

Proposition 4.3

Consider the vector bundle morphism $\bar{c}_{\pi}: \chi_{x} \in T_{G} P \otimes T^{*} X \mapsto c_{T_{G} P}\left(\overline{\mathrm{d}}_{x} \otimes \chi_{x}\right) \in T X \otimes T^{*} X$. There is a oneto-one correspondence between principal connections (splittings of the exact sequence (6)) and global sections $\chi$ of the bundle $T_{G} P \otimes T^{*} X$ for which $\bar{c}_{\pi}(\chi)=\operatorname{Id}_{T X} \in \Gamma\left(X, T X \otimes T^{*} X\right)$ holds.

\section{Definition 4.10}

We call bundle of principal connections associated to $\pi: P \rightarrow X$ and denote it by $\pi_{\mathrm{CP}}: \mathrm{CP} \rightarrow X$ the sub-bundle of elements $\chi_{x} \in T_{G} P \otimes T^{*} X$ for which $\bar{c}_{\pi}\left(\chi_{x}\right)=\operatorname{Id}_{x} \in T X \otimes T^{*} X$ holds.

As $\bar{c}_{\pi}$ is a morphism of vector bundles and $\operatorname{Id}_{T X}: X \rightarrow T X \otimes T^{*} X$ is a global section, the equation $\bar{c}_{\pi}\left(\chi_{x}\right)=\operatorname{Id}_{T X}(x)$ determines an affine sub-bundle $\mathrm{CP} \subseteq T_{G} P \otimes T^{*} X$, modelled over the vector sub-bundle $\operatorname{ker} \bar{c}_{\pi}=\operatorname{Ad} P \otimes T^{*} X \subseteq T_{G} P \otimes T^{*} X$.

Regarding the linear morphisms $c_{\pi}$ and $\bar{c}_{\pi}$ that define the connection bundle $J P \rightarrow P$ and the bundle of principal connections $\mathrm{CP} \rightarrow X$ (respectively by conditions $c_{\pi}(J)=\mathrm{Id}, \bar{c}_{\pi}(\chi)=\mathrm{Id}$ ), we have commutative diagrams, where the left side arises for any choice of $g \in G$ :

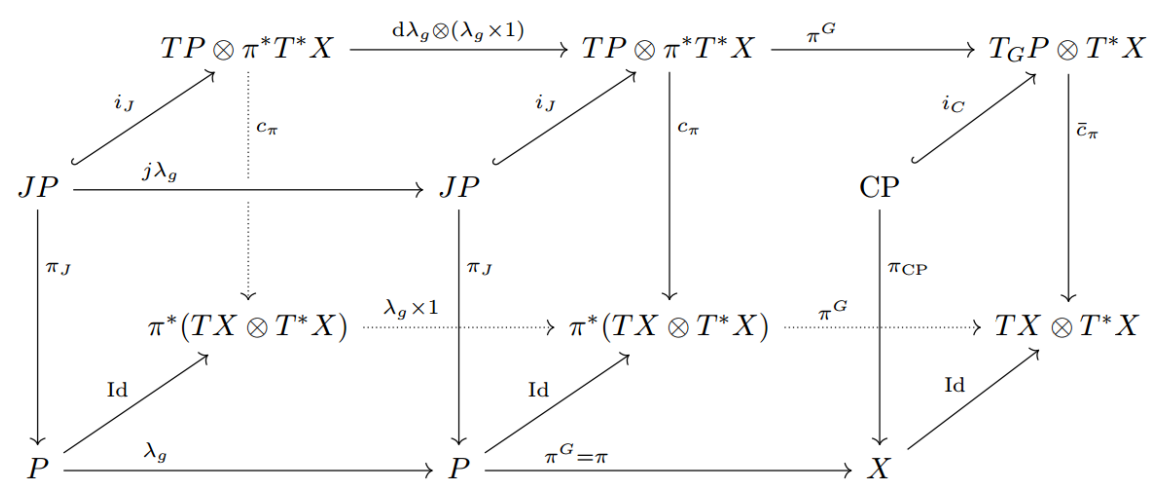

Figure 1. Commutative diagrams.

here $\pi^{G}$ denotes the quotient maps by the natural $G$-action, $i_{J}, i_{C}$ denote the natural inclusion maps, and Id denotes the identity section of the vector bundle $T X \otimes T^{*} X$.

Proposition 4.4

Consider any principal $G$-bundle $\pi: P \rightarrow X$. The bundle $\pi_{\mathrm{CP}}: \mathrm{CP} \rightarrow X$ of principal connections has a natural identification with the quotient $J P / G \rightarrow X$ of the connection bundle $J P \rightarrow P$ by the action $j \lambda_{g}$ of $G$.

Proof

From Figure 1 we see that for any element $J_{x} \in J P$, seen as $i_{J}\left(J_{x}\right) \in T P \otimes \pi^{*} T^{*} X$, its projection by $\pi^{G}$ is an element $\pi^{G}\left(i_{J}\left(J_{x}\right)\right)=\chi_{x} \in T_{G} P \otimes T^{*} X$ such that $\bar{c}_{\pi}\left(\chi_{x}\right)=\operatorname{Id}_{x}$, therefore, by definition, $\chi_{x}$ is an element contained (using $i_{C}$ ) in $C P \subset T_{G} P \otimes T^{*} X$.

Hence, $\pi^{G} \circ i_{J}$ on $J P$ factors as the composition $i_{C} \circ \bar{\pi}$, for some mapping $\bar{\pi}: J P \rightarrow C P$. This mapping, missing in diagram Figure 1, describes $\mathrm{CP}$ as a quotient manifold $J P / G$, if it is a regular projection whose fibers coincide with the $G$-orbits on $J P$. 
As the quotient manifolds are taken with respect to the action of the group $G$, in the diagram Figure 1 on each horizontal line we may state $\pi^{G} \circ\left(\mathrm{d} \lambda_{g} \otimes\left(\lambda_{g} \times 1\right)\right)=\pi^{G}, \pi^{G} \circ\left(\lambda_{g} \times 1\right)=\pi^{G}, \pi^{G} \circ \lambda_{g}=\pi^{G}$, respectively.

We shall see that the fibers of $\bar{\pi}$ are precisely the orbits in $J P$ by the $j \lambda_{g}$ action.

Indeed, if we have two elements $J_{x},\left(j \lambda_{g}\right)\left(J_{x}\right) \in J P$ on the same $G$-orbit, then

$$
i_{C} \circ \bar{\pi} \circ\left(j \lambda_{g}\right)\left(J_{x}\right)=\pi^{G} \circ i_{J} \circ\left(j \lambda_{g}\right)\left(J_{x}\right)=\pi^{G} \circ\left(\mathrm{d} \lambda_{g} \otimes\left(\lambda_{g} \times 1\right)\right) \circ i_{J}\left(J_{x}\right)=\pi^{G} \circ i_{J}\left(J_{x}\right)=i_{C} \circ \bar{\pi}\left(J_{x}\right)
$$

As $i_{C}$ is an immersion, we conclude that $\bar{\pi}\left(\left(j \lambda_{g}\right)\left(J_{x}\right)\right)=\bar{\pi}\left(J_{x}\right)$. Elements in the same $G$-orbit in $J P$ project by $\bar{\pi}$ into the same element in $\mathrm{CP}$.

Conversely, given two elements $J_{1}, J_{2} \in J P$ such that $\bar{\pi}\left(J_{1}\right)=\bar{\pi}\left(J_{2}\right)$, we have $i_{C} \circ \bar{\pi}\left(J_{1}\right)=i_{C} \circ \bar{\pi}\left(J_{2}\right)$ hence $\pi^{G} \circ i_{J}\left(J_{1}\right)=\pi^{G} \circ i_{J}\left(J_{2}\right)$, which implies $\left(\pi^{G}\right.$ is the quotient map by the group action) the existence of some element $g \in G$ with $\left(\mathrm{d} \lambda_{g} \otimes\left(\lambda_{g} \times 1\right)\right) \circ i_{J}\left(J_{1}\right)=i_{J}\left(J_{2}\right)$ and therefore $i_{J} \circ j \lambda_{g}\left(J_{1}\right)=i_{J}\left(J_{2}\right)$. As $i_{J}$ is an immersion, we conclude $j \lambda_{g}\left(J_{1}\right)=J_{2}$. Two elements that project by $\bar{\pi}$ into the same element $\chi \in \mathrm{CP}$ are, necessarily, elements in the same orbit by the action of the group $G$ on $J P$.

We conclude that $\bar{\pi}: J P \rightarrow \mathrm{CP}$ is the quotient map $\pi^{G}: J P \rightarrow J P / G$ by the group $G$ acting as $j \lambda_{g}$.

As the bundle of principal connections can be seen as a quotient bundle, the geometrical meaning of principal connections, and its relation to the general notion of connection $J \in \Gamma(J P)$ is clarified next.

\section{Proposition 4.5}

The natural mapping $J_{x} \in J P \rightarrow\left(\pi_{J}\left(J_{x}\right), \pi_{G}\left(J_{x}\right)\right) \in P \times \mathrm{CP}$ establishes a natural diffeomorphism of the connection bundle $\pi_{J}: J P \rightarrow P$ with the pull-back $\pi^{*} \mathrm{CP}=P \times_{\left(\pi, \pi_{\mathrm{CP}}\right)} \mathrm{CP} \rightarrow P$ of the bundle of principal connections $\pi_{\mathrm{CP}}: \mathrm{CP} \rightarrow X$ by $\pi: P \rightarrow X$. Moreover, the left action $j \lambda_{g}$ on $J P$ is identified in the pull-back bundle with $\lambda_{g} \times \operatorname{Id}_{\mathrm{CP}}$.

The natural projector $\pi^{G}: J P \rightarrow \mathrm{CP}$ determines a mapping that identifies the set $\Gamma^{G}(P, J P)$, of globally defined $G$-covariant connections, with the set $\Gamma(X, \mathrm{CP})$, of globally defined principal connections.

\section{Proof}

We use the morphisms in the Figure 1, now completed with the quotient morphism $\pi^{G}: J P \rightarrow \mathrm{CP}$. As $\pi \circ \pi_{J}=\pi_{\mathrm{CP}} \circ \pi^{G}$ on $J P$, it is clear that the fibered regular projector $J_{x} \in J P \rightarrow\left(\pi_{J}\left(J_{x}\right), \pi_{G}\left(J_{x}\right)\right) \in P \times \mathrm{CP}$ takes values on the sub-bundle $P \times_{\left(\pi, \pi_{\mathrm{CP}}\right)} \mathrm{CP} \rightarrow P$. It suffices to prove that the regular mapping $J_{x} \in J P \rightarrow$ $\left(\pi_{J}\left(J_{x}\right), \pi_{G}\left(J_{x}\right)\right) \in P \times_{\left(\pi, \pi_{\mathrm{CP}}\right)} \mathrm{CP}$ is bijective to prove that it is a global diffeomorphism.

Consider any element $\left(p_{x}, \chi_{x}\right) \in P \times_{X} \mathrm{CP}$. Using $i_{C}$, we get an element $\left(p_{x}, i_{C}\left(\chi_{x}\right)\right) \in P \times_{X}\left(T_{G} P \otimes T^{*} X\right)$. Using the identifications $P \times_{X} T_{G} P=T P$ and $P \times_{X} T^{*} X=\pi^{*} T^{*} X$, we get $\left(p_{x}, i_{C}\left(\chi_{x}\right)\right) \in T P \otimes \pi^{*} T^{*} X$. To prove that this element belongs to $J P \subset T P \otimes \pi^{*} T^{*} X$, it suffices to see that its image by $\pi^{G} \circ c_{\pi}$ is $\operatorname{Id}_{x} \in$ $\left(T X \otimes T^{*} X\right)_{x}$. In fact:

$$
\pi^{G}\left(c_{\pi}\left(p_{x}, i_{C}\left(\chi_{x}\right)\right)\right)=\bar{c}_{\pi}\left(\pi^{G}\left(p_{x}, i_{C}\left(\chi_{x}\right)\right)\right)=\bar{c}_{\pi}\left(i_{C}\left(\chi_{x}\right)\right)=\operatorname{Id}\left(\pi_{\mathrm{CP}}\left(\chi_{x}\right)\right)=\operatorname{Id}_{x}
$$

This proves that $\left(p_{x}, i_{C}\left(\chi_{x}\right)\right) \in J P \subset T P \otimes \pi^{*} T^{*} X$. The projection of this element by $\left(\pi_{J}, \pi^{G}\right)$ is the original element $\left(p_{x}, \chi_{x}\right) \in P \times_{X} \mathrm{CP}$, hence we have surjectivity of the mapping in our statement.

As we know $\left(\pi_{T P}, \pi^{G}\right): T P \rightarrow P \times T_{G} P$ and $i: \pi^{*} T^{*} X \hookrightarrow P \times T^{*} X$ are immersions whose images are $P \times_{X} T_{G} P=\pi^{*} T_{G} P$ and $P \times_{X} T^{*} X=\pi^{*} T^{*} X$, respectively. Therefore they determine a bundle immersion for the tensor products $T P \otimes \pi^{*} T^{*} X \rightarrow \pi^{*}\left(T_{G} P \otimes T^{*} X\right)$. The restriction to the sub-bundle $J P$ is $J_{x} \in J P \rightarrow$ $\left(\pi_{J}\left(J_{x}\right), \pi_{G}\left(J_{x}\right)\right) \in P \times_{X}\left(T_{G} P \otimes T^{*} X\right)$, which is still injective and, as we already know, has image $P \times_{X} \mathrm{CP}$. This proves our statement and allows to identify the pull-back $\pi^{*} \mathrm{CP}=P \times_{X} \mathrm{CP}$ of the bundle of principal connections with the connection bundle $J P$.

Moreover, as $\pi_{J} \circ j \lambda_{g}=\lambda_{g} \circ \pi_{J}$ and $\pi^{G} \circ j \lambda_{g}=\pi^{G}$ we may also state that the action $j \lambda_{g}$ on $J P$ is transformed into $\left(\lambda_{g}, \mathrm{Id}_{\mathrm{CP}}\right)$ on $\pi^{*} \mathrm{CP}=P \times_{X} \mathrm{CP}$

Finally, to prove the equivalence between $\Gamma^{G}(P, J P)$ with $\Gamma(X, \mathrm{CP})$, observe that any connection $J: P \rightarrow$ $J P$ such that $J \circ \lambda_{g}=j \lambda_{g} \circ J$ determines a smooth mapping on the quotient $\chi: P / G \rightarrow J P / G$, that is a principal connection $\chi \in \Gamma(X, \mathrm{CP})$. Conversely, any smooth mapping $\chi \in \Gamma(X, \mathrm{CP})$ determines an induced mapping $J=\left(\operatorname{Id}_{P}, \chi\right): \pi^{*} X=P \times_{X} X \rightarrow \pi^{*} \mathrm{CP}=P \times_{X} \mathrm{CP}$, such that $J \circ\left(\lambda_{g}, \operatorname{Id}_{X}\right)=\left(\lambda_{G}, \operatorname{Id}_{\mathrm{CP}}\right) \circ J$. Using our identification $\pi^{*} \mathrm{CP}=J P$, this is a section $J \in \Gamma(P, J P)$ covariant in the sense $J \circ \lambda_{g}=j \lambda_{g} \circ J$. 


\subsection{Reduction by closed subgroups}

The quotient manifold $J P / H$ for some subgroup $H \subseteq G$ has a more convenient expression in the trivialization given in our last proposition. In this case:

\section{Corollary 4.1}

Consider $\pi: P \rightarrow X$ a principal $G$-bundle, $H \subseteq G$ a closed subgroup, and $\pi_{H S t r}: H$ Str $=P / H \rightarrow X$ the quotient bundle (bundle of $H$-structures). The natural projectors $\pi_{J}: J P \rightarrow P$ and $\pi^{G}: J P \rightarrow \mathrm{CP}$ determine a natural identification $\left(\bar{\pi}_{J}, \bar{\pi}^{G}\right): J P / H \rightarrow H \operatorname{Str} \times{ }_{X} \mathrm{CP}$.

Variational principles for sections of the bundle $P$ are characterized by the choice of a Lagrangian density, a $\Lambda^{n} T^{*} X$-valued function on the jet bundle $J P$. Fixing a particular (non-vanishing) volume element $\operatorname{vol}_{X} \in$ $\Omega^{n}(X)=\Gamma\left(X, \Lambda^{n} T^{*} X\right)$, this section can be expressed as $\mathcal{L} \cdot \operatorname{vol}_{X}$ where $\mathcal{L}$ is a smooth function on $J P$.

\section{Definition 4.11}

Let $\pi: P \rightarrow X$ be a principal $G$-bundle. We call Lagrangian function any smooth function $\mathcal{L} \in \mathcal{C}^{\infty}(J P)$. We say a Lagrangian function is $\varphi$-invariant for some $X$-bundle automorphism $\varphi: P \rightarrow P$, if $\mathcal{L} \circ j \varphi=\mathcal{L}$. We say $\mathcal{L}$ is translation-invariant if it is invariant for all left translations $\lambda_{g}$.

As the induced action $j \varphi: J P \rightarrow J P$, for some translation morphism $\varphi=\lambda_{g}: P \rightarrow P$, is identified with $\varphi \times \operatorname{Id}$ on $P \times{ }_{X} \mathrm{CP}, \lambda_{g}$-symmetries are then more easy to deal with in the fibered product representation $P \times{ }_{X} \mathrm{CP}$ than in the jet bundle $J P$. Field variational theories on principal $G$-bundles may be trivialized, using the bundle of principal connections $\mathrm{CP}$ and the following notions:

\section{Definition 4.12}

For any Lagrangian function $\mathcal{L}(j p): J P \rightarrow \mathbb{R}$, we call associated trivialized Lagrangian $\ell$ the function $\ell(p, \chi): P \times_{X} \mathrm{CP} \rightarrow \mathbb{R}$ induced by $\mathcal{L}$, using the natural identification $J P \simeq \pi^{*} \mathrm{CP}=P \times_{X} \mathrm{CP}$. If the Lagrangian function $\mathcal{L}$ is invariant for $j \lambda_{h}$, for each translation $h \in H$ on a certain closed subgroup $H \subset G$, the values $\ell(p, \chi)$ only depend on the connection and the $H$-structure $H p \in P / H$, determining a smooth function $\ell(q, \chi): H \operatorname{Str} \times{ }_{X} \mathrm{CP} \rightarrow \mathbb{R}$, called the $H$-reduced Lagrangian associated to $\mathcal{L}$, where $\pi_{H \operatorname{Str}}: H \operatorname{Str}=P / H \rightarrow X$ is the bundle of $H$-structures and $\pi_{\mathrm{CP}}: \mathrm{CP} \rightarrow X$ the bundle of principal connections.

For the case $H=\{e\}$, the $H$-reduced Lagrangian $\ell$ is simply the trivialized Lagrangian associated to the Lagrangian function $\mathcal{L}(j p)$. For the case $H=G$, with a $G$-invariant Lagrangian $\mathcal{L}$, the associated $H$-reduced Lagrangian $\ell$ is called the reduced Lagrangian, which is a function on CP. Conversely, any smooth function $\ell(q, \chi)$ on $H \operatorname{Str} \times{ }_{X} \mathrm{CP}$ defines, by composition with $\pi^{H}: P \times_{X} \mathrm{CP} \rightarrow H \operatorname{Str} \times_{X} \mathrm{CP}$, a smooth function on $P \times_{X} \mathrm{CP}$ invariant for the action $\lambda_{h} \times \mathrm{Id}$, determining then a Lagrangian function $\mathcal{L} \in \mathcal{C}^{\infty}(J P)$ that is invariant for the action $j \lambda_{h}$, for each $h \in H$.

Regarding the behaviour of the Lagrangian, the trivialized Lagrangian, or the reduced Lagrangian, when we use other symmetries (not just $G$-translations) we get:

\section{Proposition 4.6}

Consider any $X$-bundle automorphism $\phi: P \rightarrow P$ commuting with all left translations $\lambda_{g}$ (we say $\phi$ is a gauge transformation). Consider the induced bundle morphism $j \phi: J P \rightarrow J P$. Both $\phi$ and $j \phi$ factor by translations leading to $a c_{\phi}: H \operatorname{Str} \rightarrow H$ Str on the bundle $H$ Str $=P / H$ of $H$-structures, defined by $a c_{\phi}(H p)=H(p \phi)$, $\mathrm{c} \phi: \mathrm{CP} \rightarrow \mathrm{CP}$ on the bundle of principal connections $\mathrm{CP}=J P / G$ defined by $\mathrm{c} \phi\left(\chi_{x}\right)=(\overline{\mathrm{d} \phi} \otimes 1)\left(\chi_{x}\right)$ where $\overline{\mathrm{d} \phi} \otimes 1: T_{G} P \otimes T^{*} X \rightarrow T_{G} P \otimes T^{*} X$ is induced by $\mathrm{d} \phi: T P \rightarrow T P$, and also leading to $\overline{j \phi}: J P / H \rightarrow J P / H$ on the quotient jet bundle $J P / H$, in such a way that $\overline{j \phi}$ is identified with $\left(a c_{\phi}, \mathrm{c} \phi\right)$ when we use the trivialization $J P / H \simeq H \operatorname{Str} \times{ }_{X} \mathrm{CP}$.

\section{Proof}

Taking into account that $j \phi: J P \rightarrow J P$ is determined on the affine sub-bundle $J P \subset T P \otimes \pi^{*} T^{*} X$ as the restriction of $\mathrm{d} \phi \otimes 1 \in \operatorname{End}\left(T P \otimes \pi^{*} T^{*} X\right)$, we easily get that $\overline{\mathrm{d} \phi} \otimes 1: T_{G} P \otimes T^{*} X \rightarrow T_{G} P \otimes T^{*} X$ restricts to $\mathrm{CP} \subset T_{G} P \otimes T^{*} X$ as the induced mapping $\mathrm{c} \phi: \mathrm{CP} \rightarrow \mathrm{CP}$. 
The mapping induced by $\phi$ on the quotient $P / H$ is again clear, and can be expressed as $a c_{\phi}: H p \mapsto H(p \phi)$. Finally, using the identification $J P \simeq P \times{ }_{X} \mathrm{CP}$, we may identify $j \phi$ with $(\phi, c \phi)$. As $j \lambda_{h}$ is identified with $\left(\lambda_{h}\right.$, Id on $H \operatorname{Str} \times{ }_{X} \mathrm{CP}$, we conclude that $\overline{j \phi}$ is expressed in the quotient $P / H \times{ }_{X} \mathrm{CP}$ as $\left(a c_{\phi}, \mathrm{c} \phi\right)$.

Corollary 4.2

If $\mathcal{L}: J P \rightarrow \mathbb{R}$ is $\phi$-invariant for some gauge transformation $\phi: P \rightarrow P$ and $H$-invariant (invariant for $j \lambda_{h}: J P \rightarrow$ $J P$, for each $h \in H$ ) for some closed subgroup $H \subset G$, then the associated $H$-reduced Lagrangian function $\ell: H \operatorname{Str} \times_{X} \mathrm{CP}$ is also invariant for the corresponding action $\left(a c_{\phi}, \mathrm{c} \phi\right)$.

\section{Discretization of Lagrangian densities using Forward Difference operators}

In order to introduce a discretization mechanism for all the objects that have been introduced in field theories, we go back to the notions of retraction and finite difference maps, now for a fibered manifold.

\section{Definition 5.1}

Let $\pi: Y \rightarrow X$ be a bundle and $\Delta: U_{d} \rightarrow T Y$ a FD operator on $Y$ defined on an open neighbourhood $U_{d} \subset Y \times Y$ of the diagonal (Definition 2.4). We say $\Delta$ is projectable if it is a locally defined bundle morphism from $\pi \times \pi: Y \times Y \rightarrow X \times X$ to $\mathrm{d} \pi: T Y \rightarrow T X$, covering a mapping $\Delta^{X}: U_{d}^{X}=(\pi \times \pi)\left(U_{d}\right) \rightarrow T X$.

Lemma 5.1

If $\Delta: U_{d} \rightarrow T Y$ is a FD operator on $Y$, projectable as $\Delta^{X}: U_{d}^{X} \rightarrow T X$, then $\Delta^{X}$ is also a FD operator on $X$. Moreover, if $\Delta$ is faithful so is $\Delta^{X}$.

Proof

In this situation (compare Definitions 2.4 and 2.5) we get a pair of diagrams where all the sides, except for the right hand side triangle, are known to be commutative:
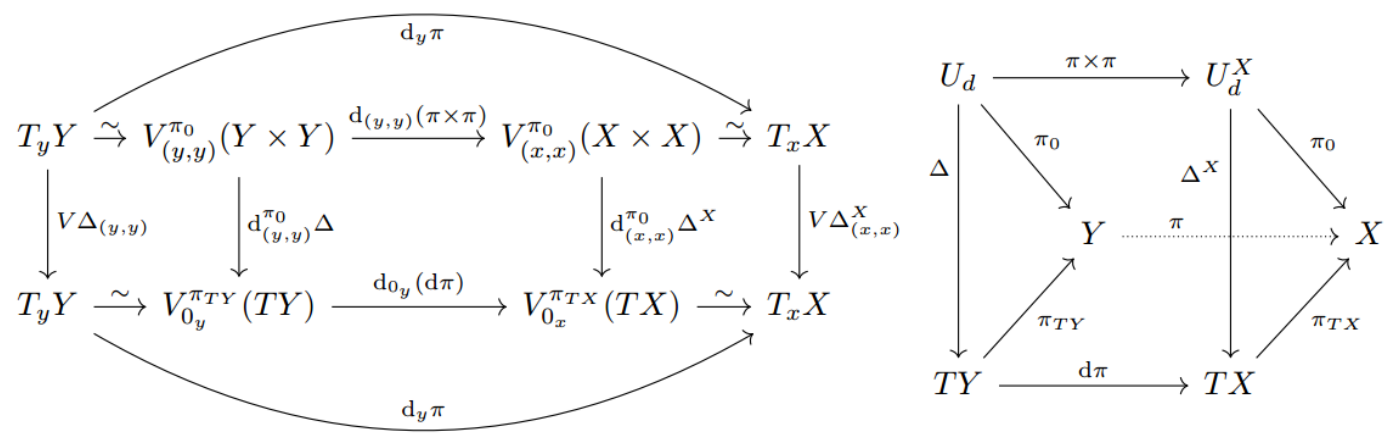

As $\pi \times \pi$ is a submersion, the image $(\pi \times \pi)\left(U_{d}\right) \subset X \times X$ is an open subset. If the smooth mapping $\Delta$ factors as $\Delta^{X}$ by the submersion, also $\Delta^{X}$ will be smooth. As $\pi \times \pi$ transforms the diagonal into the diagonal and $\mathrm{d} \pi$ the null vector into the null vector, we conclude that if $\Delta$ transforms the diagonal into the null vector so does $\Delta^{X}$. Moreover, being $\pi \times \pi$, surjective we easily verify that the right hand side triangle is also commutative, and we conclude that for a projectable FD operator the projected mapping $\Delta^{X}$ is also a FD operator on $X$. Finally, as $\mathrm{d}_{y} \pi$ is surjective we easily conclude that $V \Delta_{(y, y)}=\operatorname{Id}_{T_{y} Y}$ implies $V \Delta_{(x, x)}^{X}=\operatorname{Id}_{T_{x} X}$ and then if $\Delta$ is faithful so is $\Delta^{X}$.

We shall indicate how a projectable FD operator on a fiber bundle allows to discretize a smooth Lagrangian density, and study its particular structure in the case of $G$-invariant Lagrangian densities.

Definition 5.2

For any manifold $Y$, we denote by $Y^{\times n}$ the product $\prod_{i=0}^{i=n} Y$ of $n+1$ copies of $Y$, and denote by $\pi_{i}: Y^{\times n} \rightarrow Y$ the 
projection that maps $y^{\times n}=\left(y_{0}, \ldots, y_{n}\right) \in Y^{\times n}$ into $y_{i}$. For any bundle $\pi: Y \rightarrow X$, we denote by $\pi^{\times n}: Y^{\times n} \rightarrow$ $X^{\times n}$ the product bundle of $n+1$ copies.

For any bundle $\pi: Y \rightarrow X$, we denote by $Y^{\times_{\pi} n} \rightarrow X$ the fibered product $Y \times_{X} Y \times_{X} \ldots \times_{X} Y$ of $n+1$ copies of the bundle $\pi: Y \rightarrow X$.

Pull-back with $\pi_{i}: Y^{\times n} \rightarrow Y$ allows to construct, from any bundle $\pi_{E}: E \rightarrow Y$, a new bundle $\pi_{i}^{*} E \rightarrow Y^{\times n}$, whose fiber at a point $\left(y_{0}, \ldots, y_{n}\right) \in Y^{\times n}$ is simply $E_{y_{i}}$. Observe that the bundle $\pi_{E}^{\times n}: E^{\times n} \rightarrow Y^{\times n}$ obtained as a fibered product of $n+1$ copies of a vector bundle $\pi_{E}: E \rightarrow Y$ can be seen as a direct sum of the bundles $\pi_{i}^{*} E \rightarrow Y^{\times n}$, namely

$$
E^{\times n}=\pi_{0}^{*} E \oplus \pi_{1}^{*} E \oplus \ldots \oplus \pi_{n}^{*} E \rightarrow Y^{\times n}
$$

Consider now a FD operator $\Delta: U_{d} \rightarrow T Y$ on a manifold $Y$ (where $d_{1}(Y) \subset U_{d} \subset Y \times Y$ ). As $\Delta$ is a locally defined $Y$-bundle morphism from the bundle $\pi_{0}: Y \times Y \rightarrow Y$ to $\pi_{T Y}: T Y \rightarrow Y$, it determines, for any $n \in \mathbb{N}$, a morphism $\Delta^{\times n-1}:\left(y_{0}, y_{i}\right)_{i=1 \ldots n} \in\left(U_{d}\right)^{\times_{\pi_{0}} n-1} \mapsto\left(\Delta\left(y_{0}, y_{i}\right)\right)_{i=1 \ldots n} \in(T Y)^{\times_{\pi_{T Y}}}{ }^{n-1}$, that establishes a diffeomorphism of an open domain $\left(U_{d}\right)^{\times_{\pi_{0}} n-1}$ with an open domain $\left(U_{z}\right)^{\times_{\pi_{T Y}} n-1}$. Using the natural identifications $(Y \times Y)^{\times_{\pi_{0}} n-1}=Y \times Y^{\times n-1}=Y^{\times n}$ and $(T Y)^{\times_{\pi_{T Y}} n-1}=T Y \oplus \ldots \oplus T Y \simeq T Y \otimes \mathbb{R}^{n}$, this locally defined diffeomorphism $\Delta^{\times n-1}$ determines a map that is relevant for the discretization of jets:

\section{Definition 5.3}

For any fixed $n \in \mathbb{N}$, a FD operator $\Delta: U_{d} \rightarrow T Y$ induces the $n$-Jacobian map:

$$
J^{Y}: U_{d_{n}^{Y}} \rightarrow T Y \otimes \mathbb{R}^{n} \quad J^{Y}\left(y_{0}, \ldots, y_{n}\right)=\sum_{i=1}^{n} \Delta\left(y_{0}, y_{i}\right) \otimes e_{i}
$$

defined on the open subset $U_{d_{n}^{Y}}=\left(U_{d}\right)^{\times_{\pi_{0}}{ }^{n-1}} \subset Y^{\times n}$, neighbourhood of the diagonal $d_{n}: Y \hookrightarrow Y^{\times n}$.

We may also write $\left\langle J^{Y}\left(y^{\times n}\right), e_{i}\right\rangle=\Delta\left(y_{0}, y_{i}\right)$, where $e_{1}, \ldots, e_{n}$ stands for the natural basis on $\mathbb{R}^{n}, y_{i}=\pi_{i}\left(y^{\times n}\right)$, and $\langle\phi, e\rangle$ stands for the contraction $c_{\mathbb{R}^{n}}(\phi \otimes e)$, that is, the application of $\phi$ to a vector $e \in \mathbb{R}^{n}$ if we identify elements in $T Y \otimes \mathbb{R}^{n}$ with linear mappings $\mathbb{R}^{n} \rightarrow T Y$.

As $\Delta: U_{d} \rightarrow \Delta\left(U_{d}\right)=U_{z}$ is a diffeomorphism, the $n$-Jacobian map establishes a diffeomorphism of $U_{d_{n}^{Y}}$ with

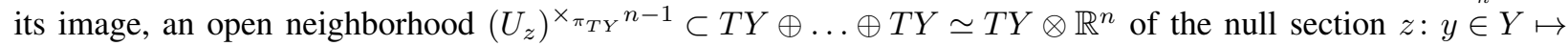
$0_{y} \in T Y \otimes \mathbb{R}^{n}$.

\section{Lemma 5.2}

If $\Delta$ is projectable to $\Delta^{X}$, then the associated Jacobian maps $J^{Y}$ and $J^{X}$ are related by:

$$
J^{X} \circ \pi^{\times n}=(\mathrm{d} \pi \otimes 1) \circ J^{Y}
$$

If $\varphi: Y \rightarrow Y$ is an automorphism on the manifold $Y$, and if $\Delta: Y \times Y \rightarrow Y$ is covariant for $\varphi$, then $J^{Y}: Y^{\times n} \rightarrow$ $T Y \otimes \mathbb{R}^{n}$ is also covariant, that is:

$$
J^{Y} \circ \varphi^{\times n}=(\mathrm{d} \varphi \otimes 1) \circ J^{Y}
$$

Proof

In the case of a projectable FD operator, we know $\mathrm{d} \pi \circ \Delta=\Delta^{X} \circ(\pi \times \pi)$, hence:

$$
\begin{gathered}
\left\langle J^{X} \circ \pi^{\times n}\left(y_{0}, \ldots, y_{n}\right), e_{i}\right\rangle=\left\langle J^{X}\left(\pi\left(y_{0}\right), \ldots, \pi\left(y_{n}\right)\right), e_{i}\right\rangle=\Delta^{X}\left(\pi\left(y_{0}\right), \pi\left(y_{i}\right)\right)=\mathrm{d} \pi\left(\Delta\left(y_{0}, y_{i}\right)\right) \\
\left\langle(\mathrm{d} \pi \otimes 1)\left(J^{Y}\left(y_{0}, \ldots, y_{n}\right)\right), e_{i}\right\rangle=\mathrm{d} \pi\left(\left\langle J^{Y}\left(y^{0}, \ldots, y^{n}\right), e_{i}\right\rangle\right)=\mathrm{d} \pi\left(\Delta\left(y_{0}, y_{i}\right)\right)
\end{gathered}
$$

thus proving our statement.

In the case that $\Delta$ is $\varphi$-covariant, we know $\mathrm{d} \varphi \circ \Delta=\Delta \circ(\varphi \times \varphi)$, and the same arguments as before changing $\Delta^{X}$ with $\Delta, J^{X}$ with $J^{Y}$ and $\pi$ with $\varphi$ proves our statement. 
One interesting aspect of the $n$-Jacobian map is the following: consider the domain $U_{d} \subset X \times X$ and the image $U_{z} \subset T X$ of some FD operator $\Delta^{X}$. Using smaller domains if needed, we may assume that $U_{x}=T_{x} X \cap U_{z}$ are convex open subsets. The inverse of the FD operator $\tau_{R}: U_{z} \subset T X \rightarrow U_{d} \subset X \times X$ transforms $0_{x_{0}} \in T_{x_{0}} X$ into $\left(x_{0}, x_{0}\right) \in X \times X$ and has the form $\left(\pi_{T X}, R\right)$ for some smooth retraction $R: U_{z} \rightarrow X$. For any choice of sequence $\left(x_{0}, x_{1}, \ldots, x_{n}\right) \in\left(U_{d}\right)^{\times_{\pi_{0}} n-1} \subset X^{\times n}$, such that $v_{i}=\Delta^{X}\left(x_{0}, x_{i}\right) \in T_{x_{0}} X$ are linearly independent vectors, we know that $R_{x_{0}}(0)=x_{0}, R_{x_{0}}\left(v_{i}\right)=x_{i}$. Hence, $R_{x_{0}}$ transforms the simplex $\left\{s_{1} v_{1}+\ldots+s_{n} v_{n}: s_{i} \geq 0, s_{1}+\ldots+\right.$ $\left.s_{n} \leq 1\right\}$ with vertices $0, v_{1}, \ldots, v_{n}$ on the linear space $T_{x_{0}} X$, into a simplicial domain $K_{\left(x_{0}, \ldots, x_{n}\right)} \subset X$ with vertices $x_{0}, x_{1}, \ldots, x_{n}$. Moreover, we know that $J_{0}=J^{X}\left(x_{0}, \ldots, x_{n}\right) \in T_{x_{0}} X \otimes \mathbb{R}^{n}$ is a linear transformation, taking the canonical basis $e_{1}, \ldots, e_{n}$ of $\mathbb{R}^{n}$ into $\Delta^{X}\left(x_{0}, x_{i}\right)=v_{i}$, hence the composition $R_{x_{0}} \circ J_{0}$ is a smooth map from $\mathbb{R}^{n}$ to $X$ transforming $e_{i}$ into $x_{i}, 0$ into $x_{0}$, and the simplex generated by $0, e_{1}, \ldots, e_{n}$ into a simplicial domain $K_{\left(x_{0}, \ldots, x_{n}\right)}$ with vertices $x_{0}, \ldots, x_{n}$.

For any density $\omega_{X} \in \Omega^{n}(X)$, its integral on the simplicial domain $K_{\left(x_{0}, \ldots, x_{n}\right)} \subset X$ can be computed using the pull-back $\omega_{\mathbb{R}^{n}}=\varphi^{*} \omega_{X} \in \Omega^{n}\left(\mathbb{R}^{n}\right)$ through $\varphi=R_{x_{0}} \circ J_{0}: \mathbb{R}^{n} \rightarrow X$ and integrating on the simplicial domain $S=\varphi^{-1}\left(K_{\left(x_{0}, \ldots, x_{n}\right)}\right) \subset \mathbb{R}^{n}$ with vertices $0, e_{1}, \ldots, e_{n}$ (with a possible sign change if $\varphi$ is not orientationpreserving). At $0 \in T_{x_{0}} X$ we know that $\mathrm{d}_{0} R_{x_{0}}: T_{x_{0}} X \rightarrow T_{x_{0}} X$ is the identity mapping. Therefore, the differential form $\omega_{\mathbb{R}^{n}}$ has a value at $0 \in \mathbb{R}^{n}$ given by $\omega_{0}=J_{0}^{*} \omega_{x_{0}} \in \Lambda^{n} \mathbb{R}^{n} \subset \Omega^{n}\left(\mathbb{R}^{n}\right)$. Any skew tensor field on $\mathbb{R}^{n}$ can be approximated by the unique translation-invariant (constant) skew tensor field with coincident value at the origin $0 \in \mathbb{R}^{n}$, and the integral of these vector fields on the simplex generated by $0, e_{1}, \ldots, e_{n}$ can be considered one approximation to another, a mechanism that represents the extension to simplices of the classical rectangle quadrature rule for integration in some interval of the real line. The integral of a constant differential form $\omega_{0}$ on the simplicial domain $S$ is simple to compute: $\frac{1}{n !} \omega_{0}\left(e_{1}, \ldots, e_{n}\right)$. For any density $\omega \in \Omega^{n}(X)$ and sequence $\left(x_{0}, \ldots, x_{n}\right) \in X^{\times n}$ near the diagonal, we have:

$$
\int_{K} \omega \simeq \pm \frac{1}{n !} \omega_{x_{0}}\left(J_{0}\left(e_{1}\right), \ldots, J_{0}\left(e_{n}\right)\right), \quad J_{0}=J^{X}\left(x_{0}, \ldots, x_{n}\right) \in T_{x_{0}} X \otimes \mathbb{R}^{n}
$$

where $K$ is a simplicial domain with vertices $x_{0}, \ldots, x_{n}$, and the sign depends on the choice of orientation on $X$ and the ordering of these vertices. The following definition is then justified:

\section{Definition 5.4}

Given a volume form $\operatorname{vol}_{X} \in \Omega^{n}(X)$ on an $n$-dimensional manifold, we call volume function induced on the bundle $T X \otimes \mathbb{R}^{n}$, the function:

$$
\operatorname{vol}^{T X \otimes \mathbb{R}^{n}}\left(J_{0}\right)=\left|\int_{S} J_{0}^{*} \operatorname{vol}_{x_{0}}\right|=\left|\frac{1}{n !} \operatorname{vol}_{x_{0}}\left(\left\langle J_{0}, e_{1}\right\rangle, \ldots,\left\langle J_{0}, e_{n}\right\rangle\right)\right|
$$

where $x_{0}=\pi_{X}\left(J_{0}\right) \in X, \operatorname{vol}_{x_{0}} \in \Lambda^{n} T_{x_{0}}^{*} X$ is the value associated to $\operatorname{vol}_{X}$ at $x_{0}, J_{0}^{*}: \Lambda^{n} T_{x_{0}}^{*} X \rightarrow \Lambda^{n} \mathbb{R}^{n}$ is the extension of $J_{0}^{*}: T_{x_{0}}^{*} X \rightarrow \mathbb{R}^{n}$ to the exterior algebra, and $S \subset \mathbb{R}^{n}$ is the simplicial domain given by $n+1$ vertices $0, e_{1}, \ldots, e_{n} \in \mathbb{R}^{n}$ (domain $S$ of points $\left(s_{1}, s_{2}, \ldots, s_{n}\right) \in \mathbb{R}^{n}$ with $s_{1}+\ldots+s_{n} \leq 1, s_{i} \geq 0$ ).

We observe that $\operatorname{vol}^{T X \otimes \mathbb{R}^{n}}\left(J_{0}\right) \neq 0$ holds precisely if $J_{0}: \mathbb{R}^{n} \rightarrow T_{x_{0}} X$ is invertible. The set of elements $J_{0} \in T X \otimes \mathbb{R}^{n}$ representing an invertible linear mapping shall be called the regular domain, or frame bundle $F_{X} \subset T X \otimes \mathbb{R}^{n} \rightarrow X$ in the bundle $T X \otimes \mathbb{R}^{n}$. The frame bundle is a dense open subset contained in $T X \otimes \mathbb{R}^{n}$.

\section{Definition 5.5}

Consider some $n$-dimensional manifold $X$ with a FD operator $\Delta^{X}: U_{d} \rightarrow T X$, and consider the associated $n$ Jacobian map $J^{X}: U_{d_{n}} \rightarrow T X \otimes \mathbb{R}^{n}$ (defined on points $\left(x_{0}, \ldots, x_{n}\right)$ such that $\left(x_{0}, x_{i}\right) \in U_{d}$ ). Points $x^{\times n} \in U_{d_{n}} \subset$ $X^{\times n}$ whose image by this map are frames (that is, points in the inverse image of the frame bundle), are called regular points in $X^{\times n}$ for the FD operator $\Delta^{X}$. Regular points are an open subset:

$$
\widetilde{X}=\left(J^{X}\right)^{-1}\left(F_{X}\right) \subset U_{d_{n}} \subset X^{\times n}
$$

In a similar way, given a projectable FD operator on a bundle $Y \rightarrow X$, for $N=\operatorname{dim} Y$, we might consider a domain of regular points $\widetilde{Y} \subset Y^{\times N}$. However, we call regular points on $Y^{\times n}$ (in this case $n=\operatorname{dim} X<\operatorname{dim} Y$ ) the open 
sub-bundle given by those elements $\left(y_{0}, \ldots, y_{n}\right)$ such that $\left(y_{0}, y_{i}\right)$ is on the domain of $\Delta$ and whose projection by $\pi^{\times n}$ is a regular point $\left(x_{0}, \ldots, x_{n}\right) \in \widetilde{X} \subset X^{\times n}$. This set of regular points is denoted by $\widetilde{J Y}$. It has a natural bundle structure $\pi^{\times n}: \widetilde{J Y} \rightarrow \widetilde{X}$.

The regular domains $\widetilde{X} \subset\left(U_{d}\right)^{\times_{T X} n-1} \subset X^{\times n}, \widetilde{J Y} \subset\left(U_{d}^{Y}\right)^{\times_{T Y} n-1} \subset Y^{\times n}$ are dense open subsets in the open neighborhoods $\left(U_{d}\right)^{\times_{T X} n-1},\left(U_{d}^{Y}\right)^{\times_{T Y} n-1}$ of the diagonals $d_{n}(X), d_{n}(Y)$, respectively. However diagonal points do not belong to these regular domains.

\section{Remark 5.1}

This generalizes to manifolds $X$ the process that leads from a line element $\mathrm{d} t$ on $\mathbb{R}$ and an interval $\left(t_{k}, t_{k+1}\right)$ to the difference value $h_{k}=t_{k+1}-t_{k}$, used in definition 3.1 to discretize reduced lagrangian mechanics.

\section{Definition 5.6}

Let $\operatorname{vol}_{X} \in \Omega^{n}(X)$ be a volume element and $\Delta^{X}$ a faithful FD operator on $X$. Consider $\tilde{X} \subset X^{\times n}$ the set of regular points for this FD operator. We call discrete volume function vol: $\widetilde{X} \rightarrow \mathbb{R}^{+}$associated to $\operatorname{vol}_{X}, \Delta^{X}$ the following:

$$
\widetilde{\operatorname{vol}}(\widetilde{x})=\operatorname{vol}^{T X \otimes \mathbb{R}^{n}}\left(J^{X}(\widetilde{x})\right)=\left|\frac{1}{n !} \operatorname{vol}_{x_{0}}\left(\Delta^{X}\left(x_{0}, x_{1}\right), \ldots, \Delta^{X}\left(x_{0}, x_{n}\right)\right)\right|, \quad \widetilde{x}=\left(x_{0}, \ldots, x_{n}\right) \in \widetilde{X} \subset X^{\times n}
$$

We still aim to discretize Lagrangian densities, not just volume forms.

\section{Definition 5.7}

For any bundle $\pi: Y \rightarrow X$, consider $\pi_{0}: X^{\times n} \rightarrow X$ and the induced bundle $\pi_{0}^{*}\left(T Y \otimes \mathbb{R}^{n}\right) \rightarrow X^{\times n}$, whose elements can be described as $\left(J_{0}, x_{1}, \ldots, x_{n}\right)$ with $J_{0} \in T Y \otimes \mathbb{R}^{n}$ and $x_{i} \in X$. Consider moreover the bundle $\pi_{0}^{*} Y \rightarrow X^{\times n}$ whose elements can be described in the form $\left(y_{0}, x_{1}, \ldots, x_{n}\right)$ for $y_{0} \in Y, x_{i} \in X$. There exists a natural bundle structure $\left(\pi^{\times n}, \pi_{0}\right): Y^{\times n} \rightarrow \pi_{0}^{*} Y \subset\left(X^{\times n}\right) \times_{\left(\pi_{0}, \pi\right)} Y$ and a natural bundle structure Id $\times \pi_{T Y \otimes \mathbb{R}^{n}}: \pi_{0}^{*}\left(T Y \otimes \mathbb{R}^{n}\right) \rightarrow \pi_{0}^{*} Y$ (induced by $\left.\pi_{T Y \otimes \mathbb{R}^{n}}: T Y \otimes \mathbb{R}^{n} \rightarrow Y\right)$.

\section{Lemma 5.3}

If $\Delta: U_{d} \rightarrow T Y$ is a projectable FD operator, the associated $n$-Jacobian map $J^{Y}$ determines a $\pi_{0}^{*} Y$-bundle injective local immersion $\left(\pi^{\times n}, J^{Y}\right): U_{d_{n}^{Y}} \subset Y^{\times n} \rightarrow \pi_{0}^{*}\left(T Y \otimes \mathbb{R}^{n}\right)$ (that we call $n$-Jacobian immersion). Its image is contained in the subset:

$$
\left\{\left(J_{0}, x_{1}, \ldots, x_{n}\right) \in \pi_{0}^{*}\left(T Y \otimes \mathbb{R}^{n}\right):\left\langle(\mathrm{d} \pi \otimes 1)\left(J_{0}\right), e_{i}\right\rangle=\Delta^{X}\left(\pi_{X}\left(J_{0}\right), x_{i}\right), \forall i=1 \ldots n\right\} \subset \pi_{0}^{*}\left(T Y \otimes \mathbb{R}^{n}\right)
$$

where $\pi_{X}=\pi \circ \pi_{T Y}: T Y \otimes \mathbb{R}^{n} \rightarrow X$ is the natural projector.

Proof

When we consider any element in the image $\left(J_{0}, x_{1}, \ldots, x_{n}\right)=\left(\pi^{\times n}, J^{Y}\right)\left(y_{0}, \ldots, y_{n}\right)$, in our definition $\Delta\left(y_{0}, y_{i}\right)=\left\langle J^{Y}\left(y_{0}, \ldots, y_{n}\right), e_{i}\right\rangle$, we may apply $\mathrm{d} \pi$ and obtain $\left\langle(\mathrm{d} \pi \otimes 1)\left(J_{0}\right), e_{i}\right\rangle=\mathrm{d} \pi\left(\Delta\left(y_{0}, y_{i}\right)\right)=$ $\Delta^{X}\left(\pi\left(y_{0}\right), \pi\left(y_{i}\right)\right)=\Delta^{X}\left(\pi_{X}\left(J_{0}\right), x_{i}\right)$. Therefore the image of $\left(\pi^{\times n}, J^{Y}\right)$ is contained in the subset given in the statement.

It is obvious that $\left(\pi^{\times n}, J^{Y}\right): Y^{\times n} \rightarrow \pi_{0}^{*}\left(T Y \otimes \mathbb{R}^{n}\right)$ respects the fibers associated to any $\left(y_{0}, x_{1}, \ldots, x_{n}\right) \in \pi_{0}^{*} Y$. It is a $Q$-bundle morphism, where $Q=\pi_{0}^{*} Y$. To prove that it is an injective local immersion it suffices to prove that, on each fiber over $q=\left(y_{0}, x_{1}, x_{2}, \ldots, x_{n}\right) \in \pi_{0}^{*} Y$, the mapping $J^{Y}$ is an injective local immersion. However, the bundles under consideration are fibered products of bundles on $Q$ :

$$
Y^{\times n}=D_{1} \times_{Q} \ldots \times_{Q} D_{n} \quad D_{i}=\pi_{i}^{*} Y, \quad\left(\pi_{i}: q \in Q \mapsto x_{i} \in X\right)
$$

Elements in $D_{i}$ can be described in the form $\left(y_{0}, x_{1}, \ldots, y_{i}, \ldots, x_{n}\right)$ where $y_{0}, y_{i} \in Y, x_{j} \in X$.

$$
\pi_{0}^{*}\left(T Y \otimes \mathbb{R}^{n}\right)=F_{1} \oplus_{Q} \cdots \oplus_{Q} F_{n}, \quad F_{i}=\pi_{0}^{*}\left(T Y \otimes \mathbb{R} e_{i}\right)
$$


Elements in $F_{i}$ can be described in the form $\left(\delta \otimes e_{i}, x_{1}, \ldots, x_{n}\right)$ where $\delta \in T Y, e_{i}$ is the $\mathrm{i}$-th vector in the canonical basis of $\mathbb{R}^{n}$ and $x_{j} \in X$.

Moreover, on the $q=\left(y_{0}, x_{1}, \ldots, x_{n}\right)$-fiber the mapping $\left(\pi^{\times n}, J^{Y}\right)$ arises from the consideration of $\varphi_{i}: y_{i} \in$ $\left(D_{i}\right)_{q} \mapsto \Delta\left(y_{0}, y_{i}\right) \otimes e_{i} \in\left(F_{i}\right)_{q}$ on each component. As we know, $\Delta$ is an injective local diffeomorphism from an open subset in $\left\{y_{0}\right\} \times Y$ to $T_{y_{0}} Y$, therefore $\Delta$ is also an injective local immersion when restricted to the $x_{i}$ fiber $\left\{y_{0}\right\} \times Y_{x_{i}}$. We conclude that each of the mappings $\varphi_{i}: Y_{x_{i}}=\left(D_{i}\right)_{q} \rightarrow\left(F_{i}\right)_{q}=T_{y_{0}} Y \otimes \mathbb{R} e_{i} \simeq T_{y_{0}} Y$ (for $i=1 \ldots n)$ are injective local immersions and consequently produce an injective local immersion $\varphi_{1} \oplus \ldots \oplus \varphi_{n}=$ $\left(\pi^{\times n}, J^{Y}\right): Y^{\times n} \rightarrow \pi_{0}^{*}\left(T Y \otimes \mathbb{R}^{n}\right)$ on an open domain of the fibered product.

Following this result, the $n$-Jacobian operator $J^{Y}$ takes values in a space somehow resembling the jet bundle, substituting vectors $e_{i}$ with vectors $\Delta^{X}\left(x_{0}, x_{i}\right)$. We can mend this situation using $J^{X}$ as follows.

On the bundle $T X \otimes \mathbb{R}^{n}$, we have the dense open subset of invertible mappings, that we called the bundle of linear frames $F_{X}$. In the same way, on the bundle $\mathbb{R}^{n} \otimes T^{*} X$ we have the dense open subset of invertible mappings, the bundle $C F_{X}$ of linear coframes. Between these open sub-bundles there exists a natural smooth morphism, the inversion morphism inv: $J \in F_{X} \mapsto J^{-1} \in C F_{X}$ defined by the condition $c_{\mathbb{R}^{n}}\left(J \otimes J^{-1}\right)=\operatorname{Id}_{T X}$. This inversion morphism transforms a given frame into its dual coframe.

\section{Definition 5.8}

We call reverse n-Jacobian map, the morphism $J_{X}^{-1}=i n v \circ J^{X}: \widetilde{X} \rightarrow C F_{X} \subset \mathbb{R}^{n} \otimes T^{*} X$. It is univocally determined by the condition $c_{\mathbb{R}^{n}}\left(J^{X} \otimes J_{X}^{-1}\right)=\operatorname{Id}_{T X}$.

For a bundle $\pi: Y \rightarrow X$ on an $n$-dimensional manifold $X$ and for any FD operator $\Delta$ on $Y$ covering $\Delta^{X}: U_{d}^{X} \rightarrow$ $T X$, if we denote by $J^{Y}, J^{X}$ the associated $n$-Jacobian maps, then $c_{T Y}\left(\mathrm{~d}_{y_{0}} \pi \otimes J^{Y}(\tilde{y})\right)=J^{X}(\tilde{x})$ (for any $\tilde{y} \in \widetilde{J Y} \subset Y^{\times n}, y_{0}=\pi_{0}(\tilde{y})$ and $\left.\tilde{x}=\pi^{\times n}(\tilde{y}) \in \widetilde{X} \subset X^{\times n}\right)$. As a consequence, the tensor $J_{X}^{Y}(\tilde{y})=c_{\mathbb{R}^{n}}\left(J^{Y}(\tilde{y}) \otimes\right.$ $\left.J_{X}^{-1}(\tilde{x})\right) \in T_{y_{0}} Y \otimes T_{\pi\left(y_{0}\right)}^{*} X$ defined for $\tilde{y} \in \widetilde{J Y}$ satisfies $c_{T Y}\left(\mathrm{~d}_{y_{0}} \pi \otimes J_{X}^{Y}(\tilde{y})\right)=\operatorname{Id}_{T_{x_{0}} X}$, so it belongs to $(J Y)_{y_{0}} \subset$ $\left(T Y \otimes \pi^{*} T^{*} X\right)_{y_{0}}$.

\section{Definition 5.9}

For a fixed bundle $Y \rightarrow X$ over an $n$-dimensional manifold $X$, and given a projectable FD operator $\Delta: U_{d} \rightarrow T Y$, we call induced forward Jacobi (FJ) operator the mapping:

$$
J_{X}^{Y}: \widetilde{J Y} \rightarrow \pi_{0}^{*}(J Y) \quad J_{X}^{Y}(\tilde{y})=\left(c_{\mathbb{R}^{n}}\left(J^{Y}(\tilde{y}) \otimes J_{X}^{-1}(\tilde{x})\right), x_{1}, \ldots, x_{n}\right)
$$

where $\tilde{x}=\pi^{\times n}(\tilde{y}), x_{i}=\pi_{i}(\tilde{x})$.

Theorem 5.1

Consider a projectable FD operator $\Delta: U_{d} \rightarrow T Y$ on a bundle $\pi: Y \rightarrow X$. Consider $\pi_{0}: X^{\times n} \rightarrow X$ the projector to the first component and $\widetilde{X} \subset X^{\times n}$ the set of regular points.

The induced FJ operator $J_{X}^{Y}: \widetilde{J Y} \rightarrow \pi_{0}^{*}(J Y)$ is an injective local diffeomorphism of $\widetilde{X}$-bundles. Moreover, for any $X$-bundle automorphism $\varphi: Y \rightarrow Y$, if $\Delta$ is covariant for $\varphi$, there holds:

$$
J_{X}^{Y} \circ \varphi^{\times n}=j \varphi \circ J_{X}^{Y}
$$

Proof

We already know (Lemma 5.3) that $\left(\pi^{\times n}, J^{Y}\right): U_{d_{n}^{Y}} \rightarrow \pi_{0}^{*}\left(T Y \otimes \mathbb{R}^{n}\right)$ is an injective local immersion, and remains so when restricted to the open domain $\widetilde{J Y} \subset U_{d_{n}^{Y}} \subset Y^{\times n}$.

On the other hand, if we restrict bundles on $X^{\times n}$ to the open subset $\widetilde{X}$ of regular points, for this restriction there exists a smooth $\widetilde{X}$-bundle morphism and its inverse:

$$
\begin{aligned}
& \left(J^{Y}, x_{1}, \ldots, x_{n}\right) \in \widetilde{X} \times_{\left(\pi_{0}, \pi_{X}\right)}\left(T Y \otimes \mathbb{R}^{n}\right) \mapsto c_{\mathbb{R}^{n}}\left(J^{Y} \otimes\left(J_{X}^{-1}\left(x_{0}, \ldots, x_{n}\right)\right)\right) \in \widetilde{X} \times_{\left(\pi_{0}, \pi_{X}\right)}\left(T Y \otimes \pi^{*} T^{*} X\right) \\
& \left(J_{X}^{Y}, x_{1}, \ldots, x_{n}\right) \in \widetilde{X} \times_{\left(\pi_{0}, \pi_{X}\right)}\left(T Y \otimes \pi^{*} T^{*} X\right) \mapsto c_{T X}\left(J_{X}^{Y} \otimes\left(J^{X}\left(x_{0}, \ldots, x_{n}\right)\right)\right) \in \widetilde{X} \times_{\left(\pi_{0}, \pi_{X}\right)}\left(T Y \otimes \mathbb{R}^{n}\right)
\end{aligned}
$$

Hence both morphisms are smooth diffeomorphisms, inverse one to the other. 
As a consequence, composing the already studied immersion with this diffeomorphism shows that, the forward Jacobi operator $J_{X}^{Y}: \widetilde{J Y} \rightarrow \pi_{0}^{*}\left(T Y \otimes T^{*} X\right)$ is an injective local immersion. We already know that it takes values on the subbundle $\pi_{0}^{*}(J Y)$.

To prove that it is an injective local diffeomorphism on its domain, it suffices to observe that $\widetilde{J Y}$ and $\pi_{0}^{*}(J Y)$ have the same dimension.

Using $n=\operatorname{dim} X, n+m=\operatorname{dim} Y$ (the fiber on $Y$ has dimension $m$ ), we know that the affine bundle $J Y \rightarrow Y$ has a fiber with dimension $\operatorname{dim} V_{y} Y \otimes T_{x} X=m \cdot n$ and $J Y \rightarrow X$ has then fiber with dimension $m+m \cdot n$. On the other hand, $\pi_{0}: X^{\times n} \rightarrow X$ has a fiber with dimension $n \cdot \operatorname{dim} X=n^{2}$, hence $\pi_{0}^{*}(J Y) \rightarrow X$ has a fiber with dimension $n^{2}+m+m n$, and as a manifold it has dimension $n+n^{2}+m+m n=(n+1) \cdot(n+m)=$ $(n+1) \cdot \operatorname{dim} Y$, which is precisely the dimension of $Y^{\times n}$ (and of its open subset $\widetilde{J Y}$ ) as a manifold.

To prove the desired covariance property, we observe that if $\varphi$ projects as $\varphi^{X}=\operatorname{Id}_{X}$ then, $j \varphi: J Y \rightarrow J Y$ is simply the restriction of $\mathrm{d} \varphi \otimes 1: T Y \otimes \pi^{*} T^{*} X \rightarrow T Y \otimes \pi^{*} T^{*} X$ to $J Y$. Therefore, the stated covariance property is a simple consequence of being $J^{Y}$ covariant (Lemma 5.2).

\section{Remark 5.2}

The $F J$ operator $J_{X}^{Y}$ generalizes to any fibered manifold $Y \rightarrow X$ the mechanism that allowed to generate an element $\chi=\frac{\tau^{-1}\left(g_{0}^{-1} g_{1}\right)}{t_{1}-t_{0}} \in \operatorname{Lie} G$ from $\left(t_{0}, g_{0}, t_{1}, g_{1}\right) \in(\mathbb{R} \times G)^{\times 1}$, used in definition 3.1 to discretize lagrangian functions in mechanics.

This results illustrates that a choice of $n+1$ elements on $Y$ is, for a certain regular family of choices, in one-toone correspondence to a choice of $n+1$ fibers (points $x_{0}, x_{1}, \ldots, x_{n}$ ) and a single jet of section on the $x_{0}$-fiber. This correspondence can be obtained fixing a projectable FD operator. A natural question now is to determine if, in a similar way to lemma 3.1, for any smooth section $y(x)$ and any regular sequence $\tilde{y}=\left(y\left(x_{0}\right), \ldots, y\left(x_{n}\right)\right)$ of $n+1$ elements on the section $y(x)$, that are close enough to a diagonal element $(y, \ldots, y) \in Y^{\times n}$, the induced elements $\left(j_{x_{0}} y, x_{1}, \ldots, x_{n}\right) \in \pi_{0}^{*}(J Y)$, and $J_{X}^{Y}(\tilde{y}) \in \pi_{0}^{*}(J Y)$, are good approximations one to another, in some sense. The intuitive affirmative answer can be misleading, as shown in the following example.

\section{Example 5.1}

Consider the bundle $\pi: Y=\mathbb{R}_{\left(y^{1}, y^{2}, y^{3}\right)}^{3} \rightarrow X=\mathbb{R}_{\left(x^{1}, x^{2}\right)}^{2}$ with the projection $x^{1}=y^{1}, x^{2}=y^{2}$. Sections of this bundle are smooth functions $y^{3}\left(x^{1}, x^{2}\right)$. We may consider on $Y$ the projectable faithful FD operator $\Delta\left(\left(y_{0}^{1}, y_{0}^{2}, y_{0}^{3}\right),\left(y_{1}^{1}, y_{1}^{2}, y_{1}^{3}\right)\right)=\left(y_{1}^{1}-y_{0}^{1}\right)\left(\partial / \partial y^{1}\right)_{y_{0}}+\left(y_{1}^{2}-y_{0}^{2}\right)\left(\partial / \partial y^{2}\right)_{y_{0}}+\left(y_{1}^{3}-y_{0}^{3}\right)\left(\partial / \partial y^{3}\right)_{y_{0}}$. Using the natural matricial notation for linear transformations, the associated 2-Jacobian map transforms any three points $y_{0}=\left(y_{0}^{1}, y_{0}^{2}, y_{0}^{3}\right), y_{1}=\left(y_{1}^{1}, y_{1}^{2}, y_{1}^{3}\right), y_{2}=\left(y_{2}^{1}, y_{2}^{2}, y_{2}^{3}\right)$ on $Y$ into:

$$
J^{Y}=\left[\begin{array}{ll}
y_{1}^{1}-y_{0}^{1} & y_{2}^{1}-y_{0}^{1} \\
y_{1}^{2}-y_{0}^{2} & y_{2}^{2}-y_{0}^{2} \\
y_{1}^{3}-y_{0}^{3} & y_{2}^{3}-y_{0}^{3}
\end{array}\right]
$$

Moreover, if the projection of these points to $\mathbb{R}^{2}$ is a non-degenerate triangle (non co-linear points on the plane $\left.\mathbb{R}_{\left(x^{1}, x^{2}\right)}^{2}\right)$, the corresponding FJ operator produces:

$$
J_{X}^{Y}=\left[\begin{array}{ll}
y_{1}^{1}-y_{0}^{1} & y_{2}^{1}-y_{0}^{1} \\
y_{1}^{2}-y_{0}^{2} & y_{2}^{2}-y_{0}^{2} \\
y_{1}^{3}-y_{0}^{3} & y_{2}^{3}-y_{0}^{3}
\end{array}\right] \cdot\left[\begin{array}{ll}
y_{1}^{1}-y_{0}^{1} & y_{2}^{1}-y_{0}^{1} \\
y_{1}^{2}-y_{0}^{2} & y_{2}^{2}-y_{0}^{2}
\end{array}\right]^{-1}
$$

As the FD operator was chosen to be faithful, for any smooth curve $y(\epsilon) \subset Y$ the curve $\Delta(y(0), y(\epsilon))$ on $T_{y(0)} Y$ takes the null value for $\epsilon=0$, and has a tangent vector at $\epsilon=0$ that gets identified with $(d / d \epsilon)_{\epsilon=0} y(\epsilon) \in T_{y_{0}} Y$. Hence, for a given smooth curve $y(\epsilon)=\left(y^{1}(\epsilon), y^{2}(\epsilon), y^{3}(\epsilon)\right)$ on $Y$, with $y(0)=y_{0} \in Y$ and for any sequence $\left(\epsilon_{k}\right)_{k \in \mathbb{N}}$ converging to zero, the sequence of tangent vectors $\frac{1}{\epsilon_{k}} \Delta\left(y_{0}, y\left(\epsilon_{k}\right)\right) \in T_{y_{0}} Y$ converge to the tangent vector $\dot{y}(0)$ of the curve $y(\epsilon)$ at $\epsilon=0$.

We might think that for any given sequence $\tilde{x}(k)=\left(x_{0}, x_{1}(k), x_{2}(k)\right)$ of non-degenerate triangles (regular points on $\left.\widetilde{X} \subset X^{\times 2}=X \times X \times X\right)$ with a common vertex $x_{0}=\left(x_{0}^{1}, x_{0}^{2}\right)$, a sequence converging to the diagonal, and for 
any given function $y(x): \mathbb{R}^{2} \rightarrow \mathbb{R}$, the associated triangles defined in $Y$ by $\left(x^{1}, x^{2}, y\left(x^{1}, x^{2}\right)\right)$ would be transformed by $J_{X}^{Y}$ into some convergent sequence on $(J Y)_{x_{0}}$ and that the limit is somehow related to the 1 -jet $j_{x_{0}} y$ associated to $y(x)$ at $x_{0}=\left(x_{0}^{1}, x_{0}^{2}\right) \in \mathbb{R}^{2}$. Next we show that this is not necessarily true.

For example, for a sequence of nondegenerate triangles $\tilde{x}(k)=\left((0,0),\left(1 / k, 1 / k^{2}\right),\left(-1 / k, 1 / k^{2}\right)\right)(k \in \mathbb{N})$, its image by some section $y:\left(x^{1}, x^{2}\right) \mapsto\left(x^{1}, x^{2}, g\left(x^{1}, x^{2}\right)\right)$ given through $g\left(x^{1}, x^{2}\right)=x^{1}+x^{2}$ or $\bar{y}$ given through $\bar{g}\left(x^{1}, x^{2}\right)=x^{1} \cdot\left(1+x^{1}\right)$ (observe that $\left.j_{(0,0)} y \neq j_{(0,0)} \bar{y}\right)$ are exactly the same sequence of triangles on $Y=\mathbb{R}^{3}$, $\tilde{y}(k)=\left((0,0,0),\left(1 / k, 1 / k^{2},(1+k) / k^{2}\right),\left(-1 / k, 1 / k^{2},(1-k) / k^{2}\right)\right)$, always lying on the linear space $y^{1}+y^{2}=$ $y^{3}$ (because the sequence of triangles was intentionally chosen to lie in $x^{2}=x^{1} \cdot x^{1}$ ). Therefore the image by the FJ operator of $\tilde{y}(k)=\bar{y}^{\times 2}(\tilde{x}(k))=y^{\times 2}(\tilde{x}(k))$ (constructed using either $g\left(x^{1}, x^{2}\right)$ or $\bar{g}\left(x^{1}, x^{2}\right)$ ) leads to the constant sequence $J_{X}^{Y}(k)=J_{X}^{Y}\left((0,0,0),\left(1 / k, 1 / k^{2},(1+k) / k^{2}\right),\left(-1 / k, 1 / k^{2},(1-k) / k^{2}\right)\right)$ on $(J Y)_{(0,0)}$ :

$$
J_{X}^{Y}(k)=\left[\begin{array}{cc}
1 & 0 \\
0 & 1 \\
1 & 1
\end{array}\right]=j_{(0,0)} y \neq j_{(0,0)} \bar{y}=\left[\begin{array}{ll}
1 & 0 \\
0 & 1 \\
1 & 0
\end{array}\right]
$$

Against our original guess, this sequence, $\tilde{y}(k)=\bar{y}^{\times 2}(\tilde{x}(k))=y^{\times 2}(\tilde{x}(k))$, does not converge to the 1-jet of $\bar{y}\left(x^{1}, x^{2}\right)=\left(x^{1}, x^{2}, x^{1}\left(1+x^{1}\right)\right)$ at $(0,0)$.

It would also be easy to construct a sequence of triangles so that, its image by $\bar{y}^{\times 2}$ defines a sequence on $\widetilde{J Y}$ converging to the diagonal point, but with an image by $J_{X}^{Y}$ that is not converging any more. It suffices a sequence of triangles that, for even terms, lie on $x^{2}=x^{1} \cdot x^{1}$, and for odd terms lie on $2 x^{2}=x^{1} \cdot x^{1}$, for example. The image by $\bar{y}^{\times 2}$ produces triangles on $\mathbb{R}^{3}$ that, for even terms lie on the plane $y^{1}+y^{2}-y^{3}=0$ and for odd terms on the plane $y^{1}+2 y^{2}-y^{3}=0$, hence its associated image by $\mathrm{FJ}$ operator oscillates between two points.

As shown in the previous example, convergence of regular points in $\widetilde{J Y}$ to some diagonal point (which does not belong to $\widetilde{J Y}$ ) does not imply convergence of the corresponding images by the FJ operator (which is only defined in $\widetilde{J Y}$ ). Let us further assume that the FD operator is faithful and that regular elements in $\widetilde{X} \subset X^{\times n}$ converging to a diagonal point $\left(x_{0}, \ldots, x_{0}\right) \in X^{\times n}$ are well chosen, in the following sense:

\section{Definition 5.10}

Let $X$ be an $n$-dimensional manifold. We call trajectory on $X^{\times n}$ coalescing to a diagonal point any curve $\alpha(\epsilon)=\left(x_{0}(\epsilon), \cdots, x_{n}(\epsilon)\right) \subset X^{\times n}$ defined on some open interval $0<\epsilon<\epsilon_{\max }$, contained in a single $\pi_{0}$-fiber (therefore $\pi_{0}(\alpha(\epsilon))=x_{0}(\epsilon)=x_{0} \in X$ does not depend on $\epsilon$ ), such that $\lim \alpha(\epsilon) \in d_{n}(X)$ for $\epsilon \rightarrow 0^{+}$(hence $\left.\lim \alpha(\epsilon)=d_{n}\left(x_{0}\right)=\left(x_{0}, \ldots, x_{0}\right)\right)$, and such that $\dot{x}_{i}(0) \in T_{x_{0}} X$ for $i=1 \ldots n$ exist and determine a frame on $T_{x_{0}} X$ (basis of tangent vectors at $x_{0}$ ).

In the presence of a trajectory coalescing to a diagonal point (in contrast with the previous example) and any faithful FD operator, we get the following fundamental property of the associated FJ operator:

\section{Theorem 5.2}

Let $\Delta: U_{d} \rightarrow T Y$ be a projectable faithful FD operator on the bundle $\pi: Y \rightarrow X$ and $J_{X}^{Y}: \widetilde{J Y} \rightarrow \pi_{0}^{*}(J Y)$ the FJ operator determined on the corresponding regular domain. Consider any smooth trajectory $\tilde{x}(\epsilon)=$ $\left(x_{0}, x_{1}(\epsilon), \ldots, x_{n}(\epsilon)\right) \subset \widetilde{X}$ contained in a single $\pi_{0}$-fiber of the regular domain $\tilde{X} \subset X^{\times n}$ and coalescing to a diagonal point.

For each smooth section $y: X \rightarrow Y$ defined on a neighbourhood of $x_{0} \in X$, there exists $\epsilon_{\max }>0$ so that, the induced smooth trajectory $\tilde{y}(\epsilon)=y^{\times n} \circ \tilde{x}(\epsilon) \subset Y^{\times n}$ belongs to the regular domain $\widetilde{J Y}$, for $0<\epsilon<\epsilon_{\max }$ and is transformed by the FJ operator into a trajectory $J_{X}^{Y}(\epsilon) \in(J Y)_{x_{0}}$, whose limit value for $\epsilon \rightarrow 0^{+}$(if it exists) coincides with $j_{x_{0}} y \in(J Y)_{x_{0}}$.

\section{Proof}

We observe first that $U_{d}$ is an open neighborhood of the diagonal, hence $\lim x_{i}(\epsilon)=x_{0}$ leads to $\lim y\left(x_{i}(\epsilon)\right)=$ $y\left(x_{0}\right)$ and therefore for some $\epsilon_{\max }$ we can assure that $\left(y\left(x_{0}\right), y\left(x_{i}(\epsilon)\right)\right) \in U_{d}$, for $0<\epsilon<\epsilon_{\max }$. As the trajectory $\tilde{x}(\epsilon)=\left(x_{0}, x_{1}(\epsilon), \ldots, x_{n}(\epsilon)\right)$ was contained in $\tilde{X}$ for $\epsilon \neq 0$, we get that $\tilde{y}(\epsilon)=y^{\times n}(\tilde{x}(\epsilon))$ is contained in the regular domain $\widetilde{J Y}$, for $0<\epsilon<\epsilon_{\max }$. 
Both $J^{X}, J^{Y}$ are smooth mappings, so they transform smooth curves $\tilde{x}(\epsilon)$ and $\tilde{y}(\epsilon)$ into smooth curves $J^{X}(\epsilon)$, $J^{Y}(\epsilon)$ in the vector spaces $T_{x_{0}} X \otimes \mathbb{R}^{n}$ and $T_{y\left(x_{0}\right)} Y \otimes \mathbb{R}^{n}$, respectively. Observe that by definition, there holds $\left\langle J_{X}^{Y}(\epsilon),\left\langle J^{X}(\epsilon), e\right\rangle\right\rangle=\left\langle J^{Y}(\epsilon), e\right\rangle$, for any $\epsilon>0$ and any $e \in \mathbb{R}^{n}$.

Denote by $y_{i}(\epsilon)=y\left(x_{i}(\epsilon)\right)$ the components of $\tilde{y}$. As $y(x)$ is smooth and $\tilde{x}(\epsilon)$ coalesces to $\left(x_{0}, \ldots, x_{0}\right)$, we get $\lim y_{i}(\epsilon)=y_{0}$ with $y_{0}=y\left(x_{0}\right)$, as $\epsilon \rightarrow 0^{+}$and $\dot{y}_{i}(0)=\left(\mathrm{d}_{x_{0}} y\right)\left(\dot{x}_{i}(0)\right)$. As the FD operator $\Delta$ is smooth and faithful we get:

$$
\begin{gathered}
\dot{y}_{i}(0)=(\mathrm{d} \Delta)_{\left(y_{0}, y_{0}\right)}\left(\dot{y}_{i}(0)\right)=\lim _{\epsilon \rightarrow 0^{+}} \frac{\Delta\left(y_{0}, y_{i}(\epsilon)\right)-\Delta\left(y_{0}, y_{0}\right)}{\epsilon}=\lim _{\epsilon \rightarrow 0^{+}} \frac{\left\langle J^{Y}(\epsilon), e_{i}\right\rangle-0}{\epsilon} \\
\dot{x}_{i}(0)=\left(\mathrm{d} \Delta^{X}\right)_{\left(x_{0}, x_{0}\right)}\left(\dot{x}_{i}(0)\right)=\lim _{\epsilon \rightarrow 0^{+}} \frac{\Delta^{X}\left(x_{0}, x_{i}(\epsilon)\right)-\Delta^{X}\left(x_{0}, x_{0}\right)}{\epsilon}=\lim _{\epsilon \rightarrow 0^{+}} \frac{\left\langle J^{X}(\epsilon), e_{i}\right\rangle-0}{\epsilon}
\end{gathered}
$$

where $\dot{x}_{i}(0) \in T_{x_{0}} X, \dot{y}_{i}(0) \in T_{y_{0}} Y$ stand for the tangent vectors at $\epsilon=0$ of the given curve $x_{i}(\epsilon)$ and its image $y_{i}(\epsilon)$.

Hence, if there exists a limit $J_{0}$ for $J_{X}^{Y}(\epsilon) \in T_{y\left(x_{0}\right)} Y \otimes T_{x_{0}}^{*} X$ as $\epsilon$ tends to zero, we have:

$$
\left\langle J_{0}, \dot{x}_{i}(0)\right\rangle=\left\langle\lim _{\epsilon \rightarrow 0^{+}} J_{X}^{Y}(\epsilon), \lim _{\epsilon \rightarrow 0^{+}} \frac{\left\langle J^{X}(\epsilon), e_{i}\right\rangle}{\epsilon}\right\rangle=\lim _{\epsilon \rightarrow 0^{+}} \frac{\left\langle J_{X}^{Y}(\epsilon),\left\langle J^{X}(\epsilon), e_{i}\right\rangle\right\rangle}{\epsilon}=\lim _{\epsilon \rightarrow 0^{+}} \frac{\left\langle J^{Y}(\epsilon), e_{i}\right\rangle}{\epsilon}=\dot{y}_{i}(0)
$$

We also know by definition that $\left\langle j_{x_{0}} y, \dot{x}_{i}(0)\right\rangle=\left(\mathrm{d}_{x_{0}} y\right)\left(\dot{x}_{i}(0)\right)=\dot{y}_{i}(0)$ for $y_{i}=y \circ x_{i}$. Therefore, both $J_{0}$ and $j_{x_{0}} y$ coincide on a certain basis of $T_{x_{0}} X$, which proves that they coincide as linear mappings from $T_{x_{0}} X$ to $T_{y\left(x_{0}\right)} Y$.

This result is analogue to the fundamental Lemma 3.1, used to discretize variational principles in geometric mechanics on Lie groups. We will not pursue a condition to warrant the existence of a limit. However, the previous result establishes, in the case of trajectories $\tilde{x}(\epsilon)$ coalescing to a diagonal point $d_{n}\left(x_{0}\right)$ in $\widetilde{X} \subset X^{\times n}$, a strong relation between the jet at $x_{0}$ associated to a section $y(x)$ and the extended trajectory $y^{\times n}(\tilde{x}(\epsilon))$ determined on $Y^{\times n}$ by the same section. This relation is given by the Forward Jacobi operator and might be exploited when one considers a family of discrete theories and its limit case when the diameter of the discretized elements tends to zero.

Definition 5.11

We call discrete Lagrangian function on a bundle $\pi: Y \rightarrow X$ any function $\mathcal{L}_{d}: U_{d_{n}^{Y}} \rightarrow \mathbb{R}$ defined on an open subset $U_{d_{n}^{Y}} \subset Y^{\times n}$ adherent to the diagonal $d_{n}^{Y}: Y \hookrightarrow Y^{\times n}$.

As the FJ operator establishes a relation between the jet bundle $J Y$ and the bundle $\widetilde{J Y} \subset Y^{\times n}$, on the corresponding regular domains, we arrive to a discretization notion for Lagrangian densities, extending to the general case the notion of discretization of lagrangian densities that was introduced for mechanics in definition 3.1:

Definition 5.12

For a fixed Lagrangian density $\mathcal{L}_{\mathrm{vol}_{X}}$ described by a smooth volume form $\operatorname{vol}_{X} \in \Omega^{n}(X)$ and a smooth function $\mathcal{L}: J Y \rightarrow \mathbb{R}$, we call associated discrete Lagrangian determined by a projectable faithful FD operator $\Delta$ on $Y$ the following function:

$$
\mathcal{L}_{d}=\left(\mathcal{L} \circ J_{X}^{Y}\right) \cdot\left(\widetilde{\operatorname{vol}} \circ \pi^{\times n}\right): \widetilde{J Y} \rightarrow \mathbb{R}
$$

where $J_{X}^{Y}$ is the FJ operator associated to $\Delta$, $\widetilde{\text { vol }}$ is defined in (9) and $\pi^{\times n}: \widetilde{J Y} \rightarrow \widetilde{X}$ is the restriction of $\pi^{\times n}: Y^{\times n} \rightarrow X^{\times n}$ to the set of regular points.

\section{Remark 5.3}

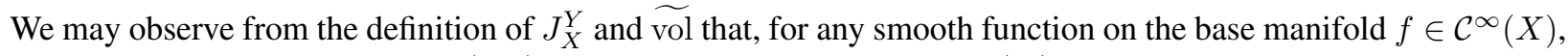
any Lagrangian function $\mathcal{L} \in \mathcal{C}^{\infty}(J Y)$ and any volume form $\operatorname{vol}_{X} \in \Omega^{n}(X)$, there holds

$$
(f \cdot \mathcal{L}) \circ J_{X}^{Y}=\left(f \circ \pi_{0}\right) \cdot\left(\mathcal{L} \circ J_{X}^{Y}\right), \quad \widetilde{f \cdot \operatorname{vol}}=\left(f \circ \pi_{0}\right) \cdot \widetilde{\operatorname{vol}}
$$

where $\pi_{0}: \widetilde{X} \rightarrow X$ is the projector to the first component. As a consequence, the discrete Lagrangian $\mathcal{L}_{d}$ defined above only depends on the particular FD operator $\Delta$ and on the Lagrangian density $\mathcal{L} \operatorname{vol}_{X}$, but not on the particular decomposition as a volume form $\operatorname{vol}_{X} \in \Omega^{n}(X)$ and a smooth function $\mathcal{L} \in \mathcal{C}^{\infty}(J Y)$. 
Following the approximation formula (8) and definitions introduced in this section, if $y \in \Gamma(Y)$ is a local section, and if $K \subset X$ is a simplicial domain with vertices $x_{0}, x_{1}, \ldots, x_{n} \in X$, ordered so that $\Delta^{X}\left(x_{0}, x_{i}\right)$ are a positively oriented basis on $T_{x_{0}} X$, we shall consider $\mathcal{L}_{d}\left(y\left(x_{0}\right), \ldots, y\left(x_{n}\right)\right) \in \mathbb{R}$ to be a good approximation of the action functional associated to $\mathcal{L}_{\mathrm{vol}_{X}}$, on the domain $K$ :

$$
\int_{K} \mathcal{L}\left(j_{x} y\right) \operatorname{vol}_{X} \simeq \mathcal{L}_{d}\left(y\left(x_{0}\right), \ldots, y\left(x_{n}\right)\right)
$$

This discretization mechanism is, furthermore, covariant:

Proposition 5.3

If $\Delta$ is a projectable faithful FD operator, covariant for some $X$-bundle automorphism $\varphi: Y \rightarrow Y$, then the discrete Lagrangian associated to $(\mathcal{L} \circ j \varphi) \cdot \operatorname{vol}_{X}$ is $\mathcal{L}_{d} \circ \varphi^{\times n}$.

In particular, if $\mathcal{L}$ is $\varphi$-invariant, the discrete Lagrangian $\mathcal{L}_{d}$ associated to the Lagrangian density $\mathcal{L} \cdot \operatorname{vol}_{X}$ is also $\varphi$-invariant.

Proof

Knowing that $\varphi$ is fibered on $\operatorname{Id}_{X}$, together with the covariance of $J_{X}^{Y}$, as stated in Theorem 5.1 leads to:

$$
\left((\mathcal{L} \circ j \varphi) \circ J_{X}^{Y}\right) \cdot\left(\widetilde{\operatorname{vol}} \circ \pi^{\times n}\right)=\left(\mathcal{L} \circ J_{X}^{Y} \circ \varphi^{\times n}\right) \cdot\left(\widetilde{\operatorname{vol}} \circ \pi^{\times n}\right)=\left(\left(\mathcal{L} \circ J_{X}^{Y}\right) \cdot\left(\widetilde{\operatorname{vol}} \circ \pi^{\times n}\right)\right) \circ \varphi^{\times n}=\mathcal{L}_{d} \circ \varphi^{\times n}
$$

Therefore, we may generate discrete variational principles, from any smooth variational principle given by a Lagrangian density, using as only tools a simplicial decomposition of the base space $X$ and a particular choice of a projectable faithful FD operator on the bundle $\pi: Y \rightarrow X$. Moreover, if the Lagrangian density is invariant for some $X$-bundle automorphisms $\varphi: Y \rightarrow Y$, the induced discrete variational principle will maintain this symmetry, when $\Delta$ is chosen to be covariant by this automorphism (for the study of variational principles, symmetries and conservation laws in discrete field theories, compare for example [10, 11, 39, 40]).

\section{A notion of parallelism on a principal bundle}

The notion of $G$-covariant projectable FD operator on a principal $G$-bundle is that of a particular smooth morphism $\Delta: P \times P \rightarrow T P$ that covariates with the diagonal action $\lambda_{g} \times \lambda_{g}$ on $P \times P$ and $\mathrm{d} \lambda_{g}$ on $T P$. This leads to the study of a quotient manifold $(P \times P) / G$, a manifold known as Ehresmann Gauge groupoid [7, 9, 30]. This section explores the geometrical structure of $(P \times P) / G$.

\subsection{The groupoid of fiber-to-fiber endomorphisms}

Definition 6.1

We call fiber-to-fiber endomorphism on the principal $G$-bundle $\pi: P \rightarrow X$, any morphism $\psi: P_{x} \rightarrow P$ whose domain is a single fiber $P_{x} \subseteq P$ and such that $\psi(g p)=g \psi(p), \forall p \in P_{x}, g \in G$. The set of all fiber-to-fiber endomorphisms is denoted by End $P$.

Covariance by the action of the whole Lie group implies that $\psi \in$ End $P$ is in fact an isomorphism of $G$-spaces, between the whole (source) fiber $P_{x_{0}}$ and a single image fiber $P_{x_{1}} \subset P$.

\section{Definition 6.2}

We call source map $s$ : End $P \rightarrow X$ the mapping that transforms an element $\psi \in$ End $P$ into its domain, a $G$-orbit $s(\psi) \in P / G$ that we may identify with a point $x_{0}=s(\psi) \in X$. We call target map $t:$ End $P \rightarrow X$ the mapping that transforms an element $\psi \in$ End $P$ into its image, a $G$-orbit $t(\psi) \in P / G$ that we may again identify with a point $x_{1}=t(\psi) \in P / G=X$. 
There is a natural groupoid structure (see [9] for introductory material on groupoids) in the set End $P$, of fiberto-fiber endomorphisms (also called Eheresmann gauge groupoid). Namely, if $s\left(\psi_{2}\right)=t\left(\psi_{1}\right)$, both mappings can be composed to get $\psi_{2} \circ \psi_{1}$, whose source is $s\left(\psi_{1}\right)$, and whose target is $t\left(\psi_{2}\right)$. Hence, $(s, t)$ : End $P \rightarrow X \times X$ is a groupoid morphism into the pair groupoid $X \times X$ (where composition is $\left(x_{1}, x_{2}\right) \circ\left(x_{0}, x_{1}\right)=\left(x_{0}, x_{2}\right)$ ). Moreover, Gau $P$, the gauge bundle, is the isotropy sub-groupoid (elements where $s(\psi)=t(\psi)$ ) associated to End $P$.

The idea of using fiber-to-fiber endomorphisms as discrete versions of the connection notion has its basis in the concept of parallel transport, and has been explored for a trivial bundle in [16, 24, 32]. We shall focus on a natural differentiable structure of the bundle $s$ : End $P \rightarrow X$ and its relation with the theory of covariant FD operators. We begin by fixing some notation, coherent with the choices made in section 4 for the gauge bundle.

For any $\psi \in \operatorname{End} P$ and any element $p \in P_{s(\psi)}$, we denote its image by $\psi(p)=p \psi \in P_{t(\psi)}$. For any (composable) pair of fiber-to-fiber endomorphisms $\left(\psi_{1}, \psi_{2}\right) \in$ End $P \times{ }_{(s, t)}$ End $P$, we denote its composition $p \in P_{s\left(\psi_{2}\right)} \mapsto \psi_{1}\left(\psi_{2}(p)\right) \in P_{t\left(\psi_{1}\right)}$ by $\psi_{1} \circ \psi_{2}$, or defining $\cdot$ as the reverse product $\psi_{2} \cdot \psi_{1}=\psi_{1} \circ \psi_{2}$, also as $\psi_{2} \cdot \psi_{1}=\psi_{2} \psi_{1}$. In this notation, the concatenation of endomorphisms represents the composition in the reverse order.

A fiber-to-fiber endomorphism $\psi: P_{x_{0}} \rightarrow P$ is totally determined if we fix the image by $\psi$ of a single element $p_{0} \in P$, namely if $p_{0} \psi=p_{1}$ then $\left(g p_{0}\right) \psi=g p_{1}$, for each element in $\left\{g p_{0}\right\}_{g \in G}=P_{x_{0}}$.

\section{Definition 6.3}

For any pair of elements $p_{0} \in P_{x_{0}}, p_{1} \in P_{x_{1}}$, we call fiber-to-fiber endomorphism induced by $\left(p_{0}, p_{1}\right)$, denoting it as $p_{0}^{-1} p_{1} \in$ End $P$, the mapping $q_{0} \in P_{x_{0}} \mapsto\left(q_{0} p_{0}^{-1}\right) p_{1} \in P$, unique fiber-to-fiber endomorphism $\psi: P_{x_{0}} \rightarrow P$ that transforms $p_{0}$ into $p_{1}$. The mapping $\pi^{G}:\left(p_{0}, p_{1}\right) \in P \times P \mapsto p_{0}^{-1} p_{1} \in$ End $P$ shall be called the gauge difference map on $P \times P$.

In the same way as the gauge group Gau $P_{x}$ associated to a fiber was denoted by $P_{x} P_{x}^{-1}$, the gauge groupoid End $P$ was denoted by Ehresmann by $P P^{-1}$ (with a reverse notation in [21]). The restriction of $\pi^{G}$ to $P \times{ }_{X} P \subset P \times P$ takes values on Gau $P \subset$ End $P$ and coincides with the gauge difference mapping defined in section 4. Our notation choice is consistent with the notation indicated in that section for gauge transformations $\phi_{x} \in$ Gau $P \subset$ End $P$ acting on elements $p_{x} \in P_{x}$. There still holds:

$$
\begin{array}{lrl}
p_{0}\left(p_{0}^{-1} p_{1}\right)=p_{1}, & \pi\left(p_{0} \psi_{01}\right)=t\left(\psi_{01}\right), & p_{0} \psi_{01}=p_{0} \Leftrightarrow \psi_{01}=\mathrm{Id}_{\pi\left(p_{0}\right)} \\
q_{0}\left(p_{0}^{-1} p_{1}\right)=\left(q_{0} p_{0}^{-1}\right) p_{1}, & (s, t)\left(p_{0}^{-1} p_{1}\right)=\left(\pi\left(p_{0}\right), \pi\left(p_{1}\right)\right), & \left(p_{0}^{-1} p_{1}\right)^{-1}=p_{1}^{-1} p_{0} \\
\left(g p_{0}\right)^{-1}\left(g p_{1}\right)=p_{0}^{-1} p_{1}, & \left(g p_{0}\right) \psi_{01}=g\left(p_{0} \psi_{01}\right), & p_{0}^{-1}\left(p_{1} \psi_{12}\right)=\left(p_{0}^{-1} p_{1}\right) \psi_{12} \\
\left(p_{0} \psi_{01}\right) \psi_{12}=p_{0}\left(\psi_{01} \psi_{12}\right), & \left(q_{0} \psi_{01}\right)\left(p_{0} \psi_{01}\right)^{-1}=q_{0} p_{0}^{-1}, & \left(\psi_{01} \psi_{12}\right)^{-1}=\psi_{12}^{-1} \psi_{01}^{-1} \\
(s, t)\left(\psi_{01} \psi_{12}\right)=\left(s\left(\psi_{01}\right), t\left(\psi_{12}\right)\right), & p_{0}^{-1} p_{1}=\operatorname{Id}_{P_{\pi\left(p_{0}\right)}} \Leftrightarrow p_{0}=p_{1}, &
\end{array}
$$

$\left(g \in G, p_{0}, q_{0}, p_{1} \in P, \psi_{01}, \psi_{12} \in\right.$ End $\left.P, s\left(\psi_{01}\right)=\pi\left(p_{0}\right)=\pi\left(q_{0}\right), t\left(\psi_{01}\right)=s\left(\psi_{12}\right)=\pi\left(p_{1}\right)\right)$

\section{Proposition 6.1}

Consider the bundle of fiber-to-fiber endomorphisms End $P$ associated to a principal $G$-bundle $\pi: P \rightarrow X$.

The mapping $\pi^{G}:\left(p_{0}, p_{1}\right) \in P \times P \rightarrow p_{0}^{-1} p_{1} \in$ End $P$ is surjective and its fibers are the $G$-orbits on $P \times P$ by the diagonal action $\lambda_{g} \times \lambda_{g}$ of the group $G$. There exists a unique smooth structure on End $P$, such that $\pi^{G}$ is a surjective submersion. This submersion factors by a bundle isomorphism $\bar{\pi}^{G}:(P \times P) / G \stackrel{\sim}{\rightarrow}$ End $P$.

\section{Proof}

The mapping $\pi^{G}$ is surjective: for any $\psi \in(\text { End } P)_{x_{0}, x_{1}}$ it suffices to take any $p \in P_{x_{0}}$ and $\bar{p}=p \psi \in P_{x_{1}}$ to obtain $\pi^{G}(p, \bar{p})=p^{-1} \bar{p}=p^{-1} p \psi=\psi$. Moreover, for any other pair $(q, \bar{q})$ defining a fiber-to-fiber endomorphism with source $P_{x_{0}}$, there holds $q=g p$ for some $g \in G$ (in which case $g=q p^{-1} \in G$ ). In this case $q^{-1} \bar{q}=\phi$ holds if and only if $\bar{q}=q \phi=(g p) \phi=g(p \phi)=g \bar{p}$, hence if and only if $(q, \bar{q})=\left(\lambda_{g} \times \lambda_{g}\right)(p, \bar{p})$ for some $g \in G$. We therefore conclude that $\pi^{G}$ is surjective and its fibers are the orbits by the diagonal action. As a consequence, there exists a unique natural bijection $(P \times P) / G \simeq$ End $P$ through which $\pi^{G}: P \times P \rightarrow$ End $P$ gets identified with the quotient mapping $\pi^{G}: P \times P \rightarrow(P \times P) / G$. 
Considering the diagonal action of $G$ by $\lambda_{g} \times \lambda_{g}$ on $P \times P$, there exists (check it in the trivial case $P=X \times G$ ) a unique smooth structure (the quotient structure) on $(P \times P) / G$ such that, the natural projection $\pi^{G}: P \times P \rightarrow$ $(P \times P) / G$ is a smooth surjective submersion. Therefore, the unique smooth structure on End $P$, for which $\pi^{G}$ is a surjective submersion, must be the quotient structure translated to End $P$ by the natural bijection given above.

Therefore, $(P \times P) / G$ (quotient by the diagonal action) exists and can be identified with End $P$, using the gauge difference mapping $\pi^{G}$. The corresponding groupoid structure and several geometrical interpretations are also considered in $[7,9,30]$. We want a better knowledge of the tangent bundle associated to End $P$.

Any fiber-to-fiber endomorphism $\psi_{01} \in(\text { End } P)_{x_{0} x_{1}}$ determines a mapping $\left(p_{0}, \bar{p}_{0}\right) \in P_{x_{0}} \times P_{x_{0}} \mapsto$ $\left(p_{0} \psi_{01}, \bar{p}_{0} \psi_{01}\right) \in P_{x_{1}} \times P_{x_{1}}$. This mapping factors in the quotient as a Lie group morphism $\operatorname{Conj}_{\psi_{01}}: \phi_{0} \in$ Gau $P_{x_{0}} \mapsto \psi_{01}^{-1} \phi_{0} \psi_{01}=\psi_{01} \circ \phi_{0} \circ \psi_{01}^{-1} \in \mathrm{Gau} P_{x_{1}}$.

\section{Definition 6.4}

We call adjoint action by $\psi \in$ End $P$ on $\operatorname{Lie} \operatorname{Gau} P_{s(\psi)}$, the linear morphism $\operatorname{Ad}_{\psi}: \operatorname{Lie} \operatorname{Gau} P_{s(\psi)} \rightarrow \operatorname{Lie} \operatorname{Gau} P_{t(\psi)}$ induced between the corresponding Lie algebras by the Lie group morphism Conj ${ }_{\psi}$. We call parallel transport by $\psi$ on $\operatorname{Ad} P_{s(\psi)}$, the linear morphism $(\psi \cdot): \operatorname{Ad} P_{s(\psi)} \rightarrow \operatorname{Ad} P_{t(\psi)}$ determined by $\operatorname{Ad}_{\psi}$, using the identifications Lie Gau $P_{x} \simeq \operatorname{Ad} P_{x}$ given in (7).

Recall that any $a \in \operatorname{Lie} \operatorname{Gau} P_{x}$ is identified with the $G$-covariant vector field $A: p_{x} \mapsto(d / d \epsilon)_{0} p_{x} \exp \epsilon a$ on $P_{x}$. When $\psi \in(\text { End } P)_{x}$ acts by the adjoint representation, we get $\exp \left(\epsilon \operatorname{Ad}_{\psi}(a)\right)=\psi^{-1}(\exp \epsilon a) \psi \in \operatorname{Gau} P_{t(\psi)}$, therefore the $G$-covariant vector field associated to $\operatorname{Ad}_{\psi}(a)$ is $p_{t(\psi)} \mapsto(d / d \epsilon)_{0} p_{t(\psi)} \psi^{-1}(\exp \epsilon a) \psi=$ $(d / d \epsilon)_{0} \psi\left(p_{t(\psi)} \psi^{-1} \exp \epsilon a\right)$. This vector field takes on any point $\psi(p)=p \psi \in P_{t(\psi)}$, the value $(\mathrm{d} \psi)\left((d / d \epsilon)_{0} p \exp \epsilon a\right)$, which is the $\mathrm{d} \psi$-image of the value on $p$ of the vector field $A \in \mathfrak{X}\left(P_{s(\psi)}\right)$ associated to $a$. As a consequence, the parallel transport $(\psi \cdot): \operatorname{Ad} P_{s(\psi)} \rightarrow \operatorname{Ad} P_{t(\psi)}$ is simply the transformation of any vertical $G$-invariant vector field $A$ on $P_{s(\psi)}$ into the corresponding vertical $G$-invariant vector field $\psi \cdot A$ on $P_{t(\psi)}$, defined by $(\psi \cdot A)_{\psi(p)}=\mathrm{d} \psi\left(A_{p}\right)$, using the diffeomorphism $\psi: P_{s(\psi)} \rightarrow P_{t(\psi)}$.

\section{Theorem 6.2}

The differential of the bundle morphism $\pi^{G}: P \times P \rightarrow$ End $P$ induces a surjective vector bundle morphism $s^{*} T_{G} P \oplus t^{*} T_{G} P \rightarrow T$ End $P$, whose kernel Sym is given by elements $(\psi, A, \psi \cdot A) \in s^{*} \operatorname{Ad} P \oplus t^{*} \operatorname{Ad} P \subset$ $s^{*} T_{G} P \oplus t^{*} T_{G} P$ with $\pi(A)=s(\psi)(\pi:$ Ad $P \rightarrow X)$.

Consequently, there exists an exact sequence:

$$
\begin{gathered}
0 \rightarrow \operatorname{Sym} \hookrightarrow s^{*} T_{G} P \oplus t^{*} T_{G} P \rightarrow T \text { End } P \rightarrow 0 \\
\text { Sym }=\left\{(\psi, A, \psi \cdot A): \psi \in \text { End } P, A \in \operatorname{Ad} P_{s(\psi)}\right\}
\end{gathered}
$$

and we may write $T$ End $P=\left(s^{*} T_{G} P \oplus t^{*} T_{G} P\right) /$ Sym.

\section{Proof}

Using the natural projections $\pi_{i}: P \times P \rightarrow P, i=0,1$ and the tangent bundle $T P \rightarrow P$, we get vector bundles $\pi_{i}^{*} T P \rightarrow P \times P$. In the same way, using $\pi_{0}: X \times X \rightarrow X$ (we maintain the same name for the first projector on $P \times P$ and $X \times X)$ and the Atiyah bundle $T_{G} P \rightarrow X$ we get a vector bundle $\pi_{0}^{*} T_{G} P \rightarrow X \times X$.

It is known that $T(P \times P)=\pi_{0}^{*} T P \oplus \pi_{1}^{*} T P$. Consider $\pi^{G}: P \times P \rightarrow$ End $P$, and the induced surjective vector bundle morphism $\mathrm{d} \pi^{G}: \pi_{0}^{*} T P \oplus \pi_{1}^{*} T P=T(P \times P) \rightarrow\left(\pi^{G}\right)^{*} T$ End $P$.

The quotient of $P \times P$ by $\lambda_{g} \times \lambda_{g}$ is End $P$, consequently the quotient of $\left(\pi^{G}\right)^{*} T$ End $P$ by $\lambda_{g} \times \lambda_{g}$ is $T$ End $P$. Observe also that the quotient of $\pi_{0}^{*} T P \oplus 0 \rightarrow P \times P$ by the action $\left(\mathrm{d} \lambda_{g}, \lambda_{g}\right)$ is precisely $s^{*} T_{G} P \rightarrow$ End $P$ (because $T P / G=T_{G} P$ and $s:$ End $P \rightarrow X$ is the projector induced by $\pi_{0}: P \times P \rightarrow P$ ).

Therefore, $\mathrm{d} \pi^{G}$ restricted to $\pi_{0}^{*} T P \oplus 0 \subset T(P \times P)$ factors by the action $\left(\mathrm{d} \lambda_{g}, \lambda_{g}\right)$ (because $\left.\mathrm{d} \lambda_{g}\left(0_{p}\right)=0_{g p}\right)$, inducing in the quotient a natural morphism $s^{*} T_{G} P \oplus 0 \rightarrow T$ End $P$.

In the same manner, $\mathrm{d} \pi^{G}$ restricted to $0 \oplus \pi_{1}^{*} T P$ factors by $\left(\lambda_{g}, \mathrm{~d} \lambda_{g}\right)$ by a natural morphism $0 \oplus t^{*} T_{G} P \rightarrow$ $T$ End $P$. 
As $\mathrm{d} \pi^{G}$ was a surjective morphism of vector bundles over $P \times P$, we conclude that it factors by an induced surjective morphism of vector bundles over $(P \times P) / G=$ End $P$ :

$$
s^{*} T_{G} P \oplus t^{*} T_{G} P \rightarrow T \text { End } P \rightarrow 0
$$

If $\operatorname{dim} X=n, \operatorname{dim} G=m$, then $\operatorname{dim} P=n+m$ and we know that $T P \rightarrow P$ has rank $n+m$, hence $T_{G} P \rightarrow X$ has rank $n+m$ and consequently $s^{*} T_{G} P \oplus t^{*} T_{G} P$ has rank $2 n+2 m$. On the other hand, $\operatorname{dim} \operatorname{End} P=\operatorname{dim}(P \times$ $P) / G=n+m+n+m-m=2 n+m$, and therefore $T$ End $P$ has rank $2 n+m$. The surjective morphism under consideration will have as kernel some $m$-rank vector bundle over End $P$. The proposed sub-bundle Sym has the needed rank. We only have to prove that these elements map to the null vector on $T$ End $P$, when we apply the morphism induced by $\pi^{G}\left(p_{0}, p_{1}\right)=p_{0}^{-1} p_{1}$ on the quotient manifold.

Fix $\psi \in \operatorname{End} P$ and $\left(p_{0}, p_{1}\right) \in P \times P$ projecting to $\psi=p_{0}^{-1} p_{1}$. For any choice of $A \in \operatorname{Ad} P_{x_{0}}$, we know that there exists a 1-parameter group of gauge transformations generated by $A$, that is, there exists $\phi_{\epsilon}: P_{x_{0}} \rightarrow P_{x_{0}}$ such that $(d / d \epsilon)_{\epsilon=0} p_{0} \phi_{\epsilon}=A_{p_{0}} \in V_{p_{0}} P$. The 1-parameter group of gauge transformations generated by $\psi \cdot A$ is, correspondingly, $\psi \circ \phi_{\epsilon} \circ \psi^{-1}=\psi^{-1} \phi_{\epsilon} \psi: P_{x_{1}} \rightarrow P_{x_{1}}$ (here we use the reverse notation for composition of elements in End $P)$. That is, $(d / d \epsilon)_{\epsilon=0} p_{1} \psi^{-1} \phi_{\epsilon} \psi=(\psi \cdot A)_{p_{1}} \in V_{p_{1}} P$.

Consider the curve $\left(p_{0} \phi_{\epsilon}, p_{1}\left(\psi \circ \phi_{\epsilon} \circ \psi^{-1}\right)\right)=\left(p_{0} \phi_{\epsilon}, p_{1} \psi^{-1} \phi_{\epsilon} \psi\right)$ on $P \times P$. This curve projects to End $P$ as:

$$
\left(p_{0} \phi_{\epsilon}\right)^{-1} p_{1} \psi^{-1} \phi_{\epsilon} \psi=\phi_{\epsilon}^{-1}\left(p_{0}^{-1} p_{1}\right) \psi^{-1} \phi_{\epsilon} \psi
$$

taking into account that $p_{0}^{-1} p_{1}=\psi$, the curve has a constant value $\psi \in$ End $P$, for each $\epsilon$.

Therefore, the tangent vector at $\epsilon=0$ to the curve $\alpha(\epsilon)=\left(p_{0} \phi_{\epsilon}, p_{1} \psi^{-1} \phi_{\epsilon} \psi\right) \subset P \times P$ is transformed by $\mathrm{d} \pi^{G}$ into the null vector $0 \in T_{\psi}$ End $P$. However, the curve under consideration has tangent vector $\left(A_{p_{0}},(\psi \cdot A)_{p_{1}}\right) \in$ $T_{p_{0}} P \oplus T_{p_{1}} P$. As was indicated, the projection into $T$ End $P$ can be done factoring by $T_{p_{0}} P \oplus T_{p_{1}} P \rightarrow\left(T_{G} P\right)_{x_{0}} \oplus$ $\left(T_{G} P\right)_{x_{1}}$. We conclude that $(A, \psi \cdot A) \in\left(T_{G} P\right)_{x_{0}} \oplus\left(T_{G} P\right)_{x_{1}}$ (which in particular is a pair of vertical invariant vector fields) projects into the null vector, no matter the choice of $A \in(\operatorname{Ad} P)_{x_{0}} \subset\left(T_{G} P\right)_{x_{0}}$.

Corollary 6.1

Consider the vertical sub-bundles $V^{s}$ End $P, V^{t}$ End $P, V^{(s, t)}$ End $P$ of the bundle $T$ End $P \rightarrow$ End $P$, corresponding to vertical tangent vectors for the projections $s$ : End $P \rightarrow X, t$ : End $P \rightarrow X$ and $(s, t)$ : End $P \rightarrow$ $X \times X$, respectively.

Denote by Skew $\rightarrow$ End $P$ the vector sub-bundle $\left\{(\psi, A,-\psi \cdot A) \in s^{*} \operatorname{Ad} P \oplus t^{*} \operatorname{Ad} P\right\} \subset s^{*} \operatorname{Ad} P \oplus t^{*} \operatorname{Ad} P$.

The inclusion maps $t^{*} T_{G} P \hookrightarrow s^{*} T_{G} P \oplus t^{*} T_{G} P, s^{*} T_{G} P \hookrightarrow s^{*} T_{G} P \oplus t^{*} T_{G} P$ and Skew $\hookrightarrow s^{*} T_{G} P \oplus t^{*} T_{G} P$ determine natural identifications:

$$
\begin{gathered}
V^{s} \text { End } P=\left(s^{*} \operatorname{Ad} P \oplus t^{*} T_{G} P\right) / \mathrm{Sym} \simeq t^{*} T_{G} P \\
V^{t} \text { End } P=\left(s^{*} T_{G} P \oplus t^{*} \operatorname{Ad} P\right) / \mathrm{Sym} \simeq s^{*} T_{G} P \\
V^{(s, t)} \text { End } P=\left(s^{*} \operatorname{Ad} P \oplus t^{*} \operatorname{Ad} P\right) / \operatorname{Sym} \simeq\left\{\begin{array}{l}
s^{*} \operatorname{Ad} P \\
t^{*} \operatorname{Ad} P \\
\text { Skew }
\end{array}\right.
\end{gathered}
$$

We call the natural identifications $V^{s}$ End $P \simeq t^{*} T_{G} P$ the target-trivialization, $V^{t}$ End $P \simeq s^{*} T_{G} P$ the source trivialization (which induce a target and a source trivialization for $V^{(s, t)}$ End $P$ in terms of $t^{*} \operatorname{Ad} P$ and $s^{*} \operatorname{Ad} P$, respectively), and call $V^{(s, t)}$ End $P \simeq$ Skew the equilibrated trivialization for the vertical bundle associated to $(s, t):$ End $P \rightarrow X \times X$.

\subsection{Parallelisms and principal connections}

A fundamental element in the theory of discrete connections was denoted as discrete Atiyah sequence in [32]. In our notation this sequence is simply:

$$
0 \rightarrow \operatorname{Gau} P \hookrightarrow \text { End } P \rightarrow X \times X \rightarrow 0
$$


This is an exact sequence of groupoids. A discrete connection was defined in [24, 32] as a $G$-equivariant subset $H$ or $\subset P \times P$ transversal to the diagonal, specifically:

$$
\{(p, p)\}_{p \in P}=d_{1}(P) \subset H o r \subset P \times P,\left.\quad(\operatorname{Id} \times \pi)\right|_{H o r}: H o r \rightarrow P \times X \text { invertible }
$$

In this situation, any pair $\left(x_{0}, x_{1}\right) \in X \times X$ on a certain open neighborhood of the diagonal $d_{1}(X)$ determines a fiber-to-fiber $G$-covariant morphism $\mathcal{H}_{\left(x_{0}, x_{1}\right)} \in \operatorname{End} P$, transforming $p_{0} \in P_{x_{0}}$ into the only element $p_{1} \in P_{x_{1}}$ such that $\left(p_{0}, p_{1}\right) \in H o r$, that is, $\left(p_{0}, p_{1}\right)=\left.(\operatorname{Id} \times \pi)\right|_{H o r} ^{-1}\left(p_{0}, x_{1}\right)$. Moreover, as $d_{1}(P) \subset H o r$, associated to $\left(x_{0}, x_{0}\right)$, one obtains $\mathcal{H}_{\left(x_{0}, x_{1}\right)}=\operatorname{Id}_{x_{0}} \in$ End $P$. Conversely, any mapping $\mathcal{H}:\left(x_{0}, x_{1}\right) \mapsto \mathcal{H}_{\left(x_{0}, x_{1}\right)} \in(\text { End } P)_{\left(x_{0}, x_{1}\right)}$, transforming the diagonal into the identity elements, determines a subset Hor in the conditions above, formed by all pairs $(p, q)$ where $q=\mathcal{H}_{\left(x, x_{1}\right)}(p)$, for $x=\pi(p)$ and all possible choices $x_{1} \in X$.

\section{Definition 6.5}

We call parallelism on $P \rightarrow X$, any smooth mapping $\mathcal{H}:\left(x_{0}, x_{1}\right) \in U_{d} \subset X \times X \mapsto \psi_{\left(x_{0}, x_{1}\right)} \in$ End $P$, defined on an open neighborhood $U_{d}$ of the diagonal $d_{1}(X) \subset U_{d} \subset X \times X$, such that:

$$
(s, t)\left(\mathcal{H}\left(x_{0}, x_{1}\right)\right)=\left(x_{0}, x_{1}\right), \quad \mathcal{H}\left(x_{0}, x_{0}\right)=\operatorname{Id}_{x_{0}}
$$

for any elements $x_{0}, x_{1} \in X$ on the corresponding domains.

\section{Remark 6.1}

Even though a simple splitting of the discrete Atiyah bundle recovers all the essential properties desired for a discrete connection notion in the case of mechanics, it does not fulfil all our needs for the case of variational field theories. Therefore, we shall denote sections of the Atiyah sequence satisfying (11) as horizontal lifts or parallelism notions, not as discrete connections.

The object that we call parallelism was called a discrete connection in [32], or groupoid connection by [30], if we assume the additional condition $\mathcal{H}\left(x_{0}, x_{1}\right)=\left(\mathcal{H}\left(x_{1}, x_{0}\right)\right)^{-1}$. However, in discrete theories where $P$ is restricted to a discrete set of nodes $V=\left\{x_{i}\right\}_{i \in I}$ chosen on $X$, each fiber $\mathrm{CP}_{x_{i}} \subset\left(T_{G} P \otimes T^{*} X\right)_{x_{i}}$ is an affine space modelled on $(V P)_{x_{i}} \otimes T_{x_{i}}^{*} X$, with dimension $(\operatorname{dim} X) \cdot(\operatorname{dim} G)$, while each fiber $(\text { End } P)_{x_{i} x_{j}} \simeq\left(P_{x_{i}} \times P_{x_{j}}\right) / G$ has $\operatorname{dimension} 2 \operatorname{dim} G-\operatorname{dim} G=\operatorname{dim} G$. If we pretend to consider one as the discretization of the other one, it is easily justified when $\operatorname{dim} X=1$, but, considering the dimensions of the bundles and the reduction procedure of FJ operator that we explain later, it becomes not so clear for theories where $\operatorname{dim} X>1$.

Calling $\mathcal{H}$ a groupoid connection as in [30] is, on the other hand, incompatible with another generally accepted notion of groupoid connection, seen in the literature on groupoids (see [4] and references therein) as a sub-bundle of $T$ End $P$ with specific particular conditions.

To avoid confusion we choose to denote $\mathcal{H}$ as a parallelism.

A parallelism determines a corresponding notion of parallel sections:

\section{Definition 6.6}

We say a local section $p: X \rightarrow P$ is $\mathcal{H}$-parallel if $p\left(x_{0}\right) \mathcal{H}\left(x_{0}, x_{1}\right)=p\left(x_{1}\right)$, for each pair $\left(x_{0}, x_{1}\right) \in X \times X$ in the corresponding domains.

Using a parallelism we may also determine, in the same sense as in [32], decompositions into horizontal and vertical components.

\section{Definition 6.7}

For a given parallelism $\mathcal{H}: U_{d} \subset X \times X \rightarrow$ End $P$, we call associated source vertical projector $\mathcal{V}^{s}$ : End $P \rightarrow$ Gau $P$ the morphism defined on $(s, t)^{-1}\left(U_{d}\right)$ by $\psi=\mathcal{V}^{s}(\psi) \cdot \mathcal{H}(s(\psi), t(\psi))$.

The source vertical projector is an $X$-bundle morphism from $s$ : End $P \rightarrow X$ to $s:$ Gau $P \rightarrow X$. Moreover, $\psi^{-1} \mathcal{V}^{s}(\psi)$ only depends on $(s(\psi), t(\psi))$, and $\psi=\mathcal{V}^{s}(\psi)$ for the case $\psi \in \operatorname{Gau} P \subset$ End $P$. In a similar way, there exists an associated target vertical projector $\mathcal{V}^{t}$ defined by $\psi=\mathcal{H}(s(\psi), t(\psi)) \cdot \mathcal{V}^{t}(\psi)$, which is an $X$-bundle morphism from $t$ : End $P \rightarrow X$ to $t$ : Gau $P \rightarrow X$. 


\section{Proposition 6.3}

For any fixed parallelism $\mathcal{H}: U_{d} \rightarrow$ End $P$, the differential $\mathrm{d} \mathcal{H}$ restricted to $s$-vertical tangent vectors, at points $(x, x)$ of the diagonal, determines a connection $\chi_{x}^{\mathcal{H}} \in \mathrm{CP}_{x}$.

\section{Proof}

For any smooth trajectory $\left\{x_{\epsilon}\right\} \subset X$, the identification $V_{\left(x_{0}, x_{0}\right)}^{s}(X \times X) \simeq T_{x_{0}} X$ identifies the tangent vector $(d / d \epsilon)_{\epsilon=0} x_{\epsilon} \in T_{x_{0}} X$ with the tangent vector $(d / d \epsilon)_{0}\left(x_{0}, x_{\epsilon}\right) \in V_{\left(x_{0}, x_{0}\right)}^{s}(X \times X)$.

For any smooth trajectory $\left\{\psi_{\epsilon}\right\} \subset(\text { End } P)_{x}$ and for any choice $p \in P_{x}$, the identification $V_{\psi_{0}}^{s}$ End $P \simeq\left(T_{G} P\right)_{x}$ identifies the tangent vector $(d / d \epsilon)_{0} \psi_{\epsilon}$ with the tangent vector $\pi^{G}\left((d / d \epsilon)_{0} p \psi_{\epsilon}\right) \in(T P)_{x} / G=\left(T_{G} P\right)_{x}$.

Consider the restriction $\mathrm{d}_{(x, x)}^{s} \mathcal{H}: V_{(x, x)}^{s}(X \times X) \simeq T_{x} X \rightarrow V_{\operatorname{Id}_{x}}^{s}$ End $P \simeq\left(T_{G} P\right)_{x}$. For any choice $p \in P_{x_{0}}$, the tangent vector $(d / d \epsilon)_{0} x_{\epsilon} \in T_{x_{0}} X$ is transformed by $\mathrm{d}_{\left(x_{0}, x_{0}\right)} \mathcal{H}$ into the tangent vector $\pi^{G}\left((d / d \epsilon)_{0} p \mathcal{H}\left(x_{0}, x_{\epsilon}\right)\right) \in$ $\left(T_{G} P\right)_{x_{0}}$. If we apply $\overline{\mathrm{d} \pi}:\left(T_{G} P\right)_{x_{0}} \rightarrow T_{x_{0}} X$, induced in the quotient by $\mathrm{d} \pi: T P \rightarrow T X$, the image of this tangent vector is $\overline{\mathrm{d} \pi} \circ \pi^{G}\left((d / d \epsilon)_{0} p \mathcal{H}\left(x_{0}, x_{\epsilon}\right)\right)=\mathrm{d} \pi\left((d / d \epsilon)_{0} p \mathcal{H}\left(x_{0}, x_{\epsilon}\right)\right)=(d / d \epsilon)_{0} \pi\left(p \mathcal{H}\left(x_{0}, x_{\epsilon}\right)\right)=$ $(d / d \epsilon)_{0} t\left(\mathcal{H}\left(x_{0}, x_{\epsilon}\right)\right)=(d / d \epsilon)_{0} x_{\epsilon}$.

As all tangent vectors $\delta_{x} \in T_{x} X$ can be written in the form $(d / d \epsilon)_{0} x_{\epsilon}$ for some trajectory, we conclude then that $\mathrm{d}_{(x, x)} \mathcal{H} \in\left(T_{G} P \otimes T^{*} X\right)_{x}$ satisfies $\overline{\mathrm{d} \pi} \circ \mathrm{d}_{(x, x)} \mathcal{H}=\mathrm{Id}$. Hence it is contained in the affine subspace $\mathrm{CP}_{x} \subset\left(T_{G} P \otimes T^{*} X\right)_{x}$.

\section{Definition 6.8}

We call principal connection $\chi^{\mathcal{H}} \in \Gamma(\mathrm{CP})$ determined by a parallelism $\mathcal{H}$ the following section of the connection bundle $\pi_{\mathrm{CP}}: \mathrm{CP} \rightarrow X$ :

$$
\chi_{x}^{\mathcal{H}}=\mathrm{d}_{(x, x)}^{s} \mathcal{H} \in \mathrm{CP}_{x} \subset\left(T_{G} P \otimes T^{*} X\right)_{x}
$$

That is, in the same manner as a local section $y(x): X \rightarrow Y$ of a bundle $\pi: Y \rightarrow X$ determines an extension to the jet bundle $j y: X \rightarrow J Y$, also a locally defined parallelism $\mathcal{H}: U_{d} \rightarrow$ End $P$ of a principal $G$-bundle $\pi: P \rightarrow X$ determines a locally defined principal connection $\chi^{\mathcal{H}}: X \rightarrow \mathrm{CP}$.

\section{Example 6.1}

Any local section $p: x \in X \mapsto p(x) \in P$ of a principal $G$-bundle induces a smooth mapping $p \times p: X \times X \rightarrow P \times$ $P$, which composed with $\pi^{G}$ determines a locally defined parallelism $\mathcal{H}_{p}:\left(x_{0}, x_{1}\right) \in X \times X \mapsto p\left(x_{0}\right)^{-1} p\left(x_{1}\right) \in$ End $P$. Moreover, as $\left(p\left(x_{0}\right)^{-1} p\left(x_{1}\right)\right)\left(p\left(x_{1}\right)^{-1} p\left(x_{2}\right)\right)=p\left(x_{0}\right)^{-1} p\left(x_{2}\right)$, this specific parallelism choice turns out to be a groupoid morphism from End $P$ with the composition mapping to the pair groupoid $X \times X$.

The local section $p$ itself is $\mathcal{H}_{p}$-parallel. The induced locally defined connection $\chi^{\mathcal{H}_{p}}$ is easy to compute for any tangent vector $\delta_{0}=(d / d \epsilon)_{0} x_{\epsilon} \in T_{x_{0}} X$ of a curve $x_{\epsilon} \subset X$ :

$$
\left\langle\chi^{\mathcal{H}_{p}}, \delta_{0}\right\rangle=\left(\mathrm{d}_{\left(x_{0}, x_{0}\right)} \mathcal{H}_{p}\right)\left(0,(d / d \epsilon)_{0} x_{\epsilon}\right)=\left(\frac{\mathrm{d}}{\mathrm{d} \epsilon}\right)_{0}\left(\mathcal{H}_{p}\left(x_{0}, x_{\epsilon}\right)\right)=\left(\frac{\mathrm{d}}{\mathrm{d} \epsilon}\right)_{0} p\left(x_{0}\right)^{-1} p\left(x_{\epsilon}\right) \in V_{I d_{x_{0}}}^{s} \text { End } P
$$

Using now the projector $\pi^{G}:\left(T_{G} P\right)_{s(\psi)} \oplus\left(T_{G} P\right)_{t(\psi)} \rightarrow T_{\psi}$ End $P$ induced by $(p, q) \in P \times P \mapsto p^{-1} q \in$ End $P$ and the identification $V_{\psi}^{s}$ End $P=\left(T_{G} P\right)_{t(\psi)}$, we get:

$$
\left\langle\chi^{\mathcal{H}_{p}}, \delta_{0}\right\rangle=\pi^{G}\left(\left(\mathrm{~d}_{x_{0}} p\right)\left(\delta_{0}\right)\right)=\pi^{G}\left(\left\langle j_{x_{0}} p, \delta_{0}\right\rangle\right) \in T_{p\left(x_{0}\right)} P / G=\left(T_{G} P\right)_{x_{0}}
$$

The expression above holds for all tangent vectors $\delta_{0} \in T P$. Hence, the parallelism $\mathcal{H}_{p}$ induced by a local section $p$ leads to a flat principal connection $\chi^{\mathcal{H}_{p}} \in \Gamma(\mathrm{CP})$, that coincides with the $G$-covariant connection $x \in X \mapsto \pi^{G}\left(j_{x} p\right) \in(J P) / G=\mathrm{CP}$ generated by $p$.

We observe that, if a local parallelism $\mathcal{H}(x, \bar{x})$ is induced by some local section $p(x)$, then the corresponding connection $\chi^{\mathcal{H}} \in \Gamma(\mathrm{CP})$ is the projection of the jet section $j p \in \Gamma(X, J P)$ in the quotient space $J P / G=\mathrm{CP}$. Anyway, there exist several locally defined smooth principal connections and discrete parallelisms that are groupoid morphisms and are not generated by any local section $p(x)$. 


\section{Example 6.2}

If we consider the two-dimensional sphere $X$ with its standard Riemannnian metric and the bundle $\pi: P \rightarrow X$ of Riemannian referentials (a principal $S O(2)$-bundle, isomorph as a manifold to $S O(3)$ ), we may construct a parallelism on $P$ by choosing, for any pair of non-antipodal points, $x_{0} \neq x_{1} \in X$, the mapping $\mathcal{H}\left(x_{0}, x_{1}\right)=$ $\psi_{\left(x_{0} x_{1}\right)}: P_{x_{0}} \rightarrow P_{x_{1}}$ that transforms any referential on $x_{0}$ into the referential on $x_{1}$, obtained by parallel transport along the unique great-circle joining both points (and taking $\mathcal{H}(x, x)=\operatorname{Id}_{P_{x}}$ for the pair $x_{0}=x_{1}=x$ ). We observe that this parallelism $\mathcal{H}: X \times X \rightarrow$ End $P$ is not globally defined, nor is induced by any local section. In fact it is not a groupoid morphism: On spherical triangles parallel transport along two edges is not compatible with parallel transport along the remaining edge. The connection associated to this parallelism is Levi-Civitta's connection for the sphere.

Theorem 6.4 (Generation of Parallelisms through principal connections and faithful FD operators)

Consider a principal connection $\chi$ on a principal $G$-bundle $\pi: P \rightarrow X$, and any faithful FD operator $\Delta^{X}: U_{d} \rightarrow$ $T X$ where $\Delta^{X}\left(U_{d}\right)=U_{z}$ is a star-shaped domain. There exists a parallelism $\mathcal{H}$, whose associated connection is $\chi$ and whose values $\mathcal{H}\left(x_{0}, x_{1}\right)$ are precisely the parallel transport morphisms $T_{x_{0} x_{1}}: P_{x_{0}} \rightarrow P_{x_{1}}$, associated to the connection $\chi$, along the curve segment $\alpha_{x_{0} x_{1}}(\epsilon)$ generated by $\Delta^{X}$, following Theorem 2.2.

Proof

Consider the principal connection $\chi \in \Gamma(\mathrm{CP})$ as a $G$-covariant section $\chi \in \Gamma\left(T P \otimes \pi^{*} T^{*} X\right)$. Consider for each $\left(x_{0}, x_{1}\right)$ the trajectory $\left\{\alpha_{x_{0} x_{1}}(\epsilon)\right\}_{0 \leq \epsilon \leq 1} \subset X$.

Using the trajectory $\alpha_{x_{0} x_{1}}$ (joining $x_{0}$ at $\epsilon=0$ to $x_{1}$ at $\epsilon=1$ and characterized by (1)) and the principal connection, we may determine a trajectory $\psi_{x_{0} x_{1}}(\epsilon)$ on End $P$ starting at $\operatorname{Id}_{x_{0}}$ at $\epsilon=0$ and solving, for some choice of $p_{0} \in P_{x_{0}}$, the following ordinary differential equation on End $P$ :

$$
\psi_{x_{0} x_{1}}(0)=\operatorname{Id}_{x_{0}}, \quad\left(\frac{d}{d \epsilon}\right)_{c} p_{0} \psi_{x_{0} x_{1}}(\epsilon)=\left\langle\chi_{p_{0} \psi_{x_{0} x_{1}}(c)},\left(\frac{d}{d \epsilon}\right)_{c} \alpha_{x_{0} x_{1}}(\epsilon)\right\rangle \in T_{p_{0} \psi_{x_{0} x_{1}}(c)} P
$$

As $\chi$ is $G$-covariant, the equation for another choice of $g p_{0} \in P_{x_{0}}$ is simply the image by $\mathrm{d} \lambda_{g}$ of this equation, leading to an equivalent equation. The equation and its unique local solution do not depend on the particular choice of $p_{0} \in P_{x_{0}}$ (it does depend on $\left.x_{0}, x_{1}\right)$. As $\chi$ is a connection and $\pi(p \psi)=t(\psi)$, the projection with $\mathrm{d} \pi$ of the differential equation shows that $t\left(\psi_{x_{0} x_{1}}(\epsilon)\right)=x_{\epsilon}$ satisfies a differential equation $(d / d \epsilon)_{c}\left(x_{\epsilon}\right)=\left(\frac{d}{d \epsilon}\right)_{c} \alpha_{x_{0} x_{1}}(\epsilon)$ with $x_{\epsilon}=x_{0}$ at $\epsilon=0$. Hence $x_{\epsilon}=\alpha_{x_{0} x_{1}}(\epsilon)$.

Then, $\psi_{x_{0} x_{1}}(\epsilon)$ is a family of fiber-to-fiber endomorphisms with a common source $x_{0}$ and a variable target $x_{\epsilon}=\alpha_{x_{0} x_{1}}(\epsilon)$. We may define $\mathcal{H}\left(x_{0}, x_{1}\right)$ to be the extremal value $\psi_{x_{0} x_{1}}(1)$ of this trajectory, for points $\left(x_{0}, x_{1}\right)$ such that the solution is defined at $\epsilon=1$.

To prove that $\psi_{x_{0} x_{1}}(\epsilon)$ is defined at $\epsilon=1$, for choices $\left(x_{0}, x_{1}\right)$ close enough to the diagonal, we just observe from the local existence theorem of solutions of any ODE, that $\psi_{x_{0} x_{1}}(\epsilon)$ is indeed defined for some interval $\epsilon \in[0, r]$, with $r$ small enough. In the case $r<1$, recall that $\alpha_{x_{0} \bar{x}_{1}}(\epsilon)=\alpha_{x_{0} x_{1}}(\epsilon r)$, for any $x_{0}, x_{1} \in X, \bar{x}_{1}=\alpha_{x_{0} x_{1}}(r)$ and $0 \leq \epsilon \leq 1$. Any point $\bar{x}_{1}$ on the trajectory $\alpha_{x_{0} x_{1}}$ generates then a new trajectory $\alpha_{x_{0} \bar{x}_{1}}$, that is, (up to reparameterization) a segment of the original one.

Differential equation (12) defining $\psi_{x_{0} \bar{x}_{1}}(\epsilon)$ uses $\alpha_{x_{0} \bar{x}_{1}}(\epsilon)$, which we may substitute with $\alpha_{x_{0} x_{1}}(\epsilon r)$ to conclude that $\psi_{x_{0} x_{1}}(\epsilon r)$ solves indeed the differential equation defining $\psi_{x_{0} \bar{x}_{1}}(\epsilon)$. This proves that $\psi_{x_{0} \bar{x}_{1}}(\epsilon)$ is defined for $\epsilon \in[0,1]$, and hence $\mathcal{H}\left(x_{0}, \bar{x}_{1}\right)$ is defined when $\left(x_{0}, \bar{x}_{1}\right)$ are close enough to the diagonal.

Moreover, this also proves that, whenever $\mathcal{H}\left(x_{0}, x_{1}\right)$ is defined, for any point $x_{c}=\alpha_{x_{0} x_{1}}(c)$ with $c \in[0,1]$ (a point that belongs to the curve segment $\alpha_{x_{0} x_{1}}$ joining $x_{0}$ and $x_{1}$ ), there holds

$$
\mathcal{H}\left(x_{0}, x_{c}\right)=\psi_{x_{0} x_{c}}(1)=\psi_{x_{0} x_{1}}(c)
$$

To prove that $\mathcal{H}$ smoothly depends on $x_{0}, x_{1}$, we just indicate it as a direct consequence of the existence of smooth solutions of smooth ODEs with initial conditions (in our case (12)), and the unicity and smooth dependence of the solution on parameters, in the case that the equation depends smoothly on certain parameters (in our case the parameters are $\left.x_{0}, x_{1}\right)$. 
The parallel transport determined by $\chi$ along any trajectory $\left\{x_{\epsilon}\right\}_{0 \leq \epsilon \leq 1} \subset X$ is a family of morphisms $T_{c}: P_{x_{0}} \rightarrow$ $P_{x_{c}}$ characterized as:

$$
T_{0}(p)=p, \quad\left(\frac{\mathrm{d}}{\mathrm{d} \epsilon}\right)_{c} T_{\epsilon}(p)=\left\langle\chi_{T_{c}(p)},(d / d \epsilon)_{c} x_{\epsilon}\right\rangle
$$

In our particular case, for the trajectory $x_{\epsilon}$ under consideration we know that $\mathcal{H}\left(x_{0}, x_{\epsilon}\right)=\psi_{x_{0} x_{\epsilon}}(1)=\psi_{x_{0} x_{1}}(\epsilon)$, where $\psi_{x_{0} x_{1}}(\epsilon)$ solves differential equation (12). As a consequence, the family of morphisms $T_{c}=\mathcal{H}\left(x_{0}, x_{c}\right)$ solve (14) and $\mathcal{H}\left(x_{0}, x_{1}\right)$ is the parallel transport indicated by our statement.

Let us finally determine the connection associated to $\mathcal{H}$. We may take the derivative with respect to $\epsilon$ at $\epsilon=0$ in equation (1) defining $\alpha_{x_{0} x_{1}}(\epsilon)$. Here, as $\Delta^{X}$ is faithful we may apply Theorem 2.2 to obtain $(d / d \epsilon)_{0} \alpha_{x_{0} x_{1}}(\epsilon)=$ $\Delta^{X}\left(x_{0}, x_{1}\right)$.

Moreover, we know from (13) that the trajectory $\psi_{x_{0} x_{1}}(\epsilon)$ defined on End $P$ can be written as $\psi_{x_{0} x_{1}}(\epsilon)=$ $\mathcal{H}\left(x_{0}, x_{\epsilon}\right)$, for the value $x_{\epsilon}=\alpha_{x_{0} x_{1}}(\epsilon)$. We get then from (12):

$$
\mathcal{H}\left(x_{0}, x_{0}\right)=\operatorname{Id}_{x_{0}}, \quad\left(\frac{d}{d \epsilon}\right)_{c} p_{0} \mathcal{H}\left(x_{0}, x_{\epsilon}\right)=\left\langle\chi_{p_{0} \mathcal{H}\left(x_{0}, x_{c}\right)},\left(\frac{d}{d \epsilon}\right)_{c} \alpha_{x_{0} x_{1}}(\epsilon)\right\rangle \in T_{p_{0} \psi_{x_{0} x_{1}}(c)} P
$$

which taking into account that $(d / d \epsilon)_{0} x_{\epsilon}=\Delta^{X}\left(x_{0}, x_{1}\right)$ and going to the quotient by $G$ leads at $c=0$ to:

$$
\left\langle\mathrm{d}_{\left(x_{0}, x_{0}\right)} \mathcal{H}, \Delta^{X}\left(x_{0}, x_{1}\right)\right\rangle=\left\langle\chi_{x_{0}}, \Delta^{X}\left(x_{0}, x_{1}\right)\right\rangle \in\left(T_{G} P\right)_{x_{0}}
$$

Taking this for all possible values $x_{1}$ such that $\left(x_{0}, x_{1}\right)$ is in the open domain of $\mathcal{H}$ and knowing that $x_{1} \in X \mapsto$ $\Delta^{X}\left(x_{0}, x_{1}\right) \in T_{x_{0}} X$ is a local diffeomorphism, from a neighborhood of $x_{0} \in X$ to a neighborhood of $0 \in T_{x_{0}} X$, shows that $\mathrm{d}_{\left(x_{0}, x_{0}\right)} \mathcal{H}=\chi_{x_{0}}$. The parallelism $\mathcal{H}$ induced by a faithful FD operator $\Delta^{X}$ and a connection $\chi$ determines a connection $\chi^{\mathcal{H}}$ that coincides with $\chi$.

\section{Reduction of forward difference operators}

Let $\pi: P \rightarrow X$ be a $G$-principal bundle. We shall focus now on FD operators $\Delta: U_{d} \subset P \times P \rightarrow T P$ that are covariant for the $\lambda_{g} \times \lambda_{g}$ and $\mathrm{d} \lambda_{g}$ actions on $P \times P$ and $T P$, respectively. The mapping $\Delta$ factors then through the quotient spaces $(P \times P) / G$ and $T P / G$. The first one has a geometrical interpretation as the Gauge groupoid of fiber-to-fiber endomorphisms End $P$, and the second one as Atiyah's bundle $T_{G} P$ of invariant vector fields.

\section{Definition 7.1}

We call reduced forward difference (RFD) operator, any injective local diffeomorphism $\Delta_{G}: U_{\bar{e}} \rightarrow T_{G} P$, defined on an open neighbourhood $U_{\bar{e}}$ of the unitary section $\bar{e}: x \in X \mapsto \operatorname{Id}_{x} \in$ End $P$, transforming this unitary section into the zero section $\bar{z}: x \in X \mapsto 0_{x} \in T_{G} P$ and covering $\operatorname{Id}_{X}$ for the bundle structures $s$ : End $P \rightarrow X$ and $\pi_{A t}: T_{G} P \rightarrow X$.

The RFD operator $\Delta_{G}$ is called projectable if it factors by the projectors $(s, t)$ : End $P \rightarrow X \times X$ and $\overline{\mathrm{d} \pi}: T_{G} P \rightarrow T X$, as a locally mapping $\Delta^{X}: U_{d} \rightarrow T X$, leading to a commutative diagram of locally defined morphisms:

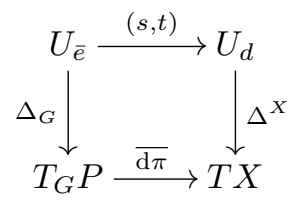

Observe that the injective local diffeomorphism condition implies that $\Delta_{G}$ is a diffeomorphism of an open neighbourhood $U_{\bar{e}} \subset$ End $P$, of the unitary section, with an open neighbourhood $U_{\bar{z}} \subset T_{G} P$, of the null section.

When $\Delta_{G}$ is an $X$-bundle morphism for the projectors $s, \pi_{A t}$, the injectivity is equivalent to injectivity on each fiber, and the local diffeomorphism condition means that the vertical differential (for the source fibration), $\left(\mathrm{d} \Delta_{G}\right)_{\psi}: V_{\psi}^{s}($ End $P) \rightarrow V_{\Delta_{G}(\psi)}^{\pi_{A t}}\left(T_{G} P\right)$, should be a vector bundle isomorphism. We may use the natural 
identifications $V_{\psi}^{s}($ End $P) \simeq\left(T_{G} P\right)_{t(\psi)}$ and $V_{a_{x}}^{\pi_{A t}}\left(T_{G} P\right) \simeq\left(T_{G} P\right)_{x}$ to express this vertical component as a linear morphism $\left(V \Delta_{G}\right)_{\psi}:\left(T_{G} P\right)_{t(\psi)} \rightarrow\left(T_{G} P\right)_{s(\psi)}$ :

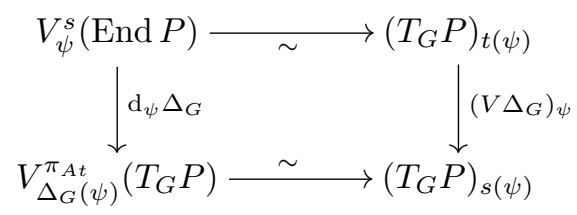

\section{Definition 7.2}

We say the RFD operator $\Delta_{G}$ is faithful if at each of the unitary elements $\operatorname{Id}_{x} \in \operatorname{End} P$, its differential on $s$-vertical vectors $\left(V \Delta_{G}\right)_{\operatorname{Id}_{x}}:\left(T_{G} P\right)_{x} \rightarrow\left(T_{G} P\right)_{x}$ is the identity map.

\section{Example 7.1}

If we consider any local $X$-bundle diffeomorphism $D: U_{\bar{e}} \rightarrow T_{G} P$ (such that $\pi_{A t} \circ D=s$ ), defined on a neighborhood $U_{\bar{e}} \subset$ End $P$ of the unitary section, we may obtain an $X$-bundle diffeomorphism that takes $\operatorname{Id}_{x} \in$ End $P$ into $0_{x} \in T_{G} P$ just by considering $\Delta_{G}(\psi)=D(\psi)-D\left(\operatorname{Id}_{s(\psi)}\right)$. As the term $D\left(\operatorname{Id}_{s(\psi)}\right)$ only depends on the source associated to $\psi$, the $s$-vertical component of the differential of this mapping, at any $\psi \in$ End $P$ is just $(V D)_{\psi}:\left(T_{G} P\right)_{t(\psi)} \rightarrow\left(T_{G} P\right)_{s(\psi)}$, invertible because $D$ is a local diffeomorphism. Therefore $\Delta_{G}$ is a local diffeomorphism and establishes a RFD operator on some open neighborhood of the identity section.

This RFD operator can be transformed into a faithful one just taking $\Delta_{G}(\psi)=(V D)_{\operatorname{Id}_{s(\psi)}}^{-1}\left(D(\psi)-D\left(\operatorname{Id}_{s(\psi)}\right)\right)$.

Recall that, for any principal $G$-bundle $\pi: P \rightarrow X$ and for its Atiyah bundle $\pi_{A t}: T_{G} P \rightarrow X$, there holds $\pi^{*} T_{G} P \simeq T P$ and that in this identification the action $\mathrm{d} \lambda_{g}$ on $T P$ gets identified with $\left(\lambda_{g}, \mathrm{Id}\right)$ on $P \times_{\left(\pi, \pi_{A t}\right)} T_{G} P$, while the projector $P \times_{\left(\pi, \pi_{A t}\right)} T_{G} P \rightarrow T_{G} P$ gets identified with $\overline{\mathrm{d} \pi}: T P \rightarrow T_{G} P$. In a similar way we observe:

\section{Proposition 7.1}

There exists a natural identification of the pull-back $\pi^{*}$ End $P$ of the bundle $s$ : End $P \rightarrow X$ by $\pi: P \rightarrow X$ with the pair bundle $\pi_{0}: P \times P \rightarrow P$.

The diagonal action $\lambda_{g} \times \lambda_{g}$ on $P \times P$ gets then identified with $\lambda_{g} \times$ Id on $P \times_{(\pi, s)}$ End $P$ and the projector $P \times{ }_{(\pi, s)}$ End $P \rightarrow$ End $P$ gets identified with $\pi^{G}: P \times P \rightarrow$ End $P$.

For any gauge transformation $\phi: P \rightarrow P$, the action $\phi \times \phi$ gets identified on the bundle $\pi^{*}$ End $P=$ $P \times{ }_{(\pi, s)}$ End $P$ with the action $\phi \times \operatorname{Conj}_{\phi}$, where we define:

$$
\operatorname{Conj}_{\phi}(\psi)=\phi_{t(\psi)} \circ \psi \circ \phi_{s(\psi)}^{-1}, \quad \psi \in \text { End } P
$$

\section{Proof}

The identification is given by $\left(p_{0}, p_{1}\right) \in P \times P \mapsto\left(p_{0}, p_{0}^{-1} p_{1}\right) \in P \times_{(\pi, s)}$ End $P$, and its inverse is defined by $\left(p_{0}, \psi\right) \in P \times_{(\pi, s)}$ End $P \mapsto\left(p_{0}, p_{0} \psi\right) \in P \times P$. The projector $\pi^{G}:\left(p_{0}, p_{1}\right) \mapsto p_{0}^{-1} p_{1}$ is then clearly identified with the projector to the End $P$-component. The actions $\lambda_{g} \times \lambda_{g}$ and $\phi \times \phi$ on $P \times P$ are clearly transformed into the actions given in our statement.

\section{Proposition 7.2}

Consider a RFD operator $\Delta_{G}: U_{\bar{e}} \rightarrow T_{G} P$ for the principal $G$-bundle $\pi: P \rightarrow X$. Consider the morphism $\Delta: U_{d}^{P} \rightarrow T P$ induced on $\pi^{*} U_{\bar{e}} \subset \pi^{*}$ End $P \simeq P \times P, \pi^{*} T_{G} P \simeq T P$. Then,

- $\Delta$ is a $G$-covariant FD operator on the bundle $P$, and the bundle morphism induced on the quotient coincides with $\Delta_{G}$.

- $\Delta$ is $\phi$-covariant for some gauge transformation $\phi: P \rightarrow P$ if and only if $\Delta_{G}$ is $\phi$-covariant (that is, $\left.\Delta_{G} \circ \operatorname{Conj}_{\phi}=\overline{\mathrm{d} \phi} \circ \Delta_{G}\right)$.

- $\Delta_{G}$ is projectable to $\Delta^{X}$ if and only if $\Delta$ is projectable to $\Delta^{X}$.

- $\Delta_{G}$ is faithful if and only if $\Delta$ is faithful. 
Proof

As $\Delta: U_{d}^{P} \rightarrow T P$ is given from $\operatorname{Id} \times \Delta_{G}: P \times_{(\pi, s)} U_{\bar{e}} \rightarrow P \times_{\left(\pi, \pi_{A t}\right)} T_{G} P$, using the identifications $P \times P \simeq$ $P \times_{(\pi, s)}$ End $P$ and $T P \simeq P \times_{\left(\pi, \pi_{A t}\right)} T_{G} P$, and $\Delta_{G}$ is an injective local diffeomorphism, also $\Delta$ is an injective local diffeomorphism. Moreover, as $\Delta_{G}$ transforms $\operatorname{Id}_{x}$ into $0_{x}$, then $\Delta$ transforms $(p, p) \in P \times P$ into $0_{p} \in T_{p} P$.

Clearly $\pi^{*} U_{\bar{e}}=P \times_{(\pi, s)} U_{\bar{e}}$ is invariant by $\lambda_{g} \times \mathrm{Id}$, therefore the domain $U_{d}^{P} \subset P \times P$ is invariant by $\lambda_{g} \times \lambda_{g}$. Moreover, as Id $\times \Delta_{G}$ is covariant when we consider the action $\lambda_{g} \times \operatorname{Id}$ on $P \times \operatorname{End} P$ and $\lambda_{g} \times \operatorname{Id}$ on $P \times T_{G} P$, we conclude that $\Delta$ is covariant when we consider the associated actions $\lambda_{g} \times \lambda_{g}$ on $P \times P$ and $\mathrm{d} \lambda_{G}$ on TP. Hence $\Delta$ is $G$-covariant.

Moreover, in the quotient by $\lambda_{g} \times \operatorname{Id}$ acting on $P \times_{(\pi, s)}$ End $P$ and on $P \times_{\left(\pi, \pi_{A t}\right)} T_{G} P$, the morphism Id $\times \Delta_{G}$ clearly projects to $\Delta_{G}$. Consequently, $\Delta: U_{d}^{P} \rightarrow T P$ projects as $\Delta_{G}$. Hence, $\Delta$ is a covariant FD operator that, in the quotient by the $G$-action coincides with $\Delta_{G}$.

Stating that $\Delta_{G} \circ \phi_{\text {End }}=\overline{\mathrm{d} \phi} \circ \Delta_{G}$ is equivalent to stating that $\left(\operatorname{Id} \times \Delta_{G}\right) \circ\left(\phi \times \phi_{\text {End }}\right)=(\phi \times \overline{\mathrm{d} \phi}) \circ\left(\operatorname{Id} \times \Delta_{G}\right)$. Using the identifications $\operatorname{Id} \times \Delta_{G}$ with $\Delta$, this is equivalent to stating that $\Delta \circ(\phi \times \phi)=\mathrm{d} \phi \circ \Delta$. Therefore, $\phi$ covariance of $\Delta$ is equivalent to $\phi$-covariance of $\Delta_{G}$.

For fixed $\Delta_{G}:$ End $P \rightarrow T_{G} P, \Delta^{X}: X \times X \rightarrow X$ (the mappings are locally defined but we shall use a lighter notation and will not keep track of the corresponding domains) consider the diagram:

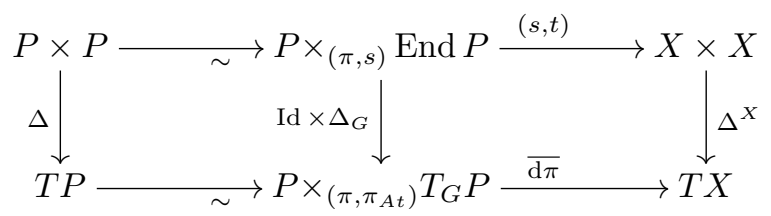

where the square on the left is commutative (because of the definition of $\Delta$ ). The square on the right, on the other hand, is commutative if and only if $\overline{\mathrm{d} \pi} \circ \Delta_{G}=(s, t) \circ \Delta^{X}$, which happens precisely if and only if the diagram in (15) is commutative. Therefore $\Delta$ is projectable to $\Delta^{X}$ if and only if $\Delta_{G}$ is projectable to $\Delta^{X}$.

Regarding the condition that $\Delta$ is faithful, observe the diagram of morphisms of vector bundles on $P_{x}$ :

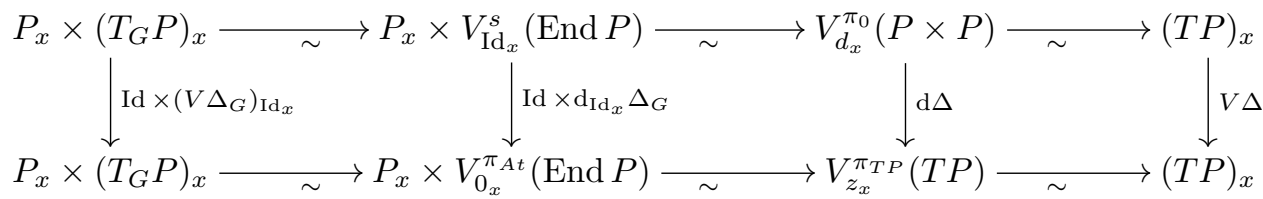

where the bundle $V_{d_{x}}^{\pi_{0}}(P \times P) \rightarrow P_{x}$ is the restriction of $V^{\pi_{0}}(P \times P) \rightarrow P \times P$ to the diagonal $d_{x}: P_{x} \hookrightarrow$ $P_{x} \times P_{x}$, the bundle $V_{z_{x}}^{\pi_{T P}}(T P) \rightarrow P_{x}$ is the restriction of $V^{\pi_{T P}}(T P) \rightarrow T P$ to the zero section $z_{x}: P_{x} \hookrightarrow(T P)_{x}$ and $(T P)_{x}$ is seen as a vector bundle on the diagonal $d\left(P_{x}\right) \subset P_{x} \times P_{x}$. Consequently, $\mathrm{d} \Delta, V \Delta$ stand for the corresponding restrictions to these sub-bundles.

The left and right hand side cycles are commutative because of the definition of $V \Delta_{G}$ and $V \Delta$. The cycle at the middle is commutative because $\Delta$ is identified with $\operatorname{Id} \times \Delta_{G}$ by our isomorphisms.

Observing now that both on the upper row or on the lower row the composition of isomorphisms is simply the natural identification $P_{x} \times\left(T_{G} P\right)_{x} \simeq(T P)_{x}$, this commutative diagram proves that $\left(V \Delta_{G}\right)_{\mathrm{Id}_{x}}$ is the identity if and only if $V \Delta_{(p, p)}$ is the identity, for any $p \in P_{x}$. Therefore $\Delta$ is faithful precisely when $\Delta_{G}$ is faithful.

\section{Remark 7.1}

We know that a covariant faithful projectable FD operator $\Delta: U_{d}^{P} \rightarrow T P$ on a principal $G$-bundle induces a faithful projectable RFD operator $\Delta_{G}: U_{\bar{e}} \rightarrow T_{G} P$. Its projection $\Delta^{X}: U_{d} \rightarrow T X$ is another faithful FD operator on the base manifold $X$, and its restriction to $U_{\bar{e}}^{\text {Gau }}=U_{\bar{e}} \cap$ Gau $P \subset$ End $P$ determines a mapping $\Delta_{\text {Gau }}: U_{\bar{e}}^{\text {Gau }} \rightarrow T_{G} P$. Moreover, considering that $\overline{\mathrm{d} \pi} \circ \Delta_{G}=\Delta^{X} \circ(s, t)$, we may conclude that $\overline{\mathrm{d} \pi}\left(\Delta_{G}\left(\phi_{x}\right)\right)=\Delta^{X}(x, x)=0_{x}$, for any 
$\phi_{x} \in$ Gau $P_{x}$. Hence, $\Delta_{\text {Gau }}$ takes values on $\operatorname{Ad} P \subset T_{G} P$, leading to the following commutative diagram:

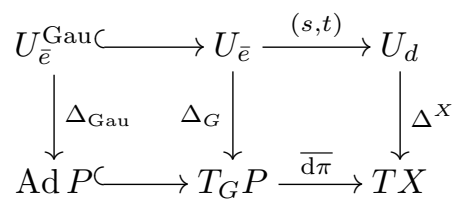

As $\Delta_{G}$ was an injective local diffeomorphism, its restriction is still an injective local immersion of Gau $P$ into Ad $P$. Taking into account the dimension, it is an injective local diffeomorphism transforming $\operatorname{Id}_{x}$ into $0_{x}$, and has a locally defined inverse that, in the case that $\Delta_{G}$ is faithful, represents a retraction map of the gauge bundle, as given in definition 7.3.

\section{Definition 7.3}

We call retraction map of the gauge bundle any smooth $X$-bundle morphism $\tau: U \rightarrow$ Gau $P$, defined on an open subset $U \subset \operatorname{Ad} P$, such that its restriction to each fiber $\tau_{x}: U_{0_{x}}=U \cap \operatorname{Ad} P_{x} \rightarrow \operatorname{Gau} P_{x}$ is a reduced retraction map on the Lie group Gau $P_{x}$ (according to Definition 2.1), using the natural identification $\operatorname{Ad} P_{x} \simeq \operatorname{Lie}$ Gau $P_{x}$ in (7), for each Lie group Gau $P_{x}$.

Being an $X$-bundle morphism and demanding that on each fiber it is a retraction map, implies that $\tau$ is an injective local diffeomorphism, identifying an open neighbourhood $U_{0}$ of the null section $0: X \rightarrow \operatorname{Ad} P$ with an open neighbourhood $U_{\bar{e}}$ of the unitary section $\bar{e}: X \rightarrow$ Gau $P$.

The exponential map exp: Ad $P \rightarrow$ Gau $P$ is a simple example of faithful retraction map of the gauge bundle. A local inverse of $\Delta_{\text {Gau }}: U_{\bar{e}}^{\text {Gau }} \rightarrow$ Ad $P$ defined from a faithful projectable RFD operator will also be an example of faithful retraction map of the gauge bundle.

The situation shown in (16) can be reversed to generate a faithful RFD operator:

Theorem 7.3 (Generation of faithful RFD operators)

For any faithful FD operator $\Delta^{X}: U_{d} \rightarrow T X\left(U_{d} \subset X \times X\right)$, any reduced retraction map $\tau: U \rightarrow$ Gau $P$ of the Gauge bundle $(U \subset \operatorname{Ad} P)$, and any parallelism $\mathcal{H}: U_{d}^{\prime} \rightarrow$ End $P\left(U_{d}^{\prime} \subset X \times X\right)$, the following expression is a (locally defined) faithful RFD operator, projectable to $\Delta^{X}$, whose restriction to Gau $P$ is the inverse $\tau^{-1}$ of the retraction map.

$$
\Delta_{G}(\psi)=\tau^{-1}\left(\mathcal{V}^{s}(\psi)\right)+\left\langle\chi_{s(\psi)}^{\mathcal{H}}, \Delta^{X}(s(\psi), t(\psi))\right\rangle
$$

where we use the connection $\chi_{x}^{\mathcal{H}}=\left(\mathrm{d}_{(x, x)} \mathcal{H}\right)$ and the source vertical projector $\mathcal{V}^{s}(\psi)=\psi \cdot(\mathcal{H}(s(\psi), t(\psi)))^{-1}$ associated to $\mathcal{H}$.

As a particular case, we may simply fix a faithful FD operator $\Delta^{X}$ and a fixed principal connection $\chi$. Then, a faithful RFD operator on $P$ can be obtained using the exponential $\operatorname{Ad} P \rightarrow \operatorname{Gau} P$ as retraction map and the parallelism $\mathcal{H}$ induced by $\Delta^{X}, \chi$ as described in Theorem 6.4. The simplest case, for a trivial principal $G$-bundle, arises from the consideration of the flat principal connection given by the trivialization, and of the retraction map of the gauge bundle, generated from a retraction map on the Lie group $G$ using the trivializations $P=X \times G$, Ad $P=X \times \operatorname{Lie} G$.

Proof

Consider the locally defined inverse $\tau^{-1}: U_{\bar{e}} \subset \operatorname{Gau} P \rightarrow U_{0} \subset \operatorname{Ad} P$ of the given retraction map $\tau$. As we know, the parallelism allows, in a neighbourhood of the identity section, to decompose elements $\psi \in$ End $P$ into horizontal and vertical components, so we may write:

$$
\psi=\tau\left(A_{\psi}\right) \cdot \mathcal{H}(s(\psi), t(\psi)), \quad A_{\psi}=\tau^{-1}\left(\mathcal{V}^{s}(\psi)\right) \in \operatorname{Ad} P_{s(\psi)}, \quad \mathcal{V}^{s}(\psi)=\psi \cdot(\mathcal{H}(s(\psi), t(\psi)))^{-1}
$$

Consider, for each $\psi \in$ End $P$ the trajectory $\left\{x_{\epsilon}\right\}_{0 \leq \epsilon \leq 1}$ on $X$ that joins $x_{0}=s(\psi)$ to $x_{1}=t(\psi)$, following Theorem 2.2. Consider $A: \psi \in$ End $P \rightarrow A_{\psi} \in \operatorname{Ad} P$ defined as $A_{\psi}=\tau^{-1}\left(\mathcal{V}^{s}(\psi)\right)$. We shall define $\Delta_{G}(\psi)$ to be the tangent vector at $\epsilon=0$ of the trajectory $\sigma_{\psi}(\epsilon)=\tau\left(\epsilon A_{\psi}\right) \cdot \mathcal{H}\left(x_{0}, x_{\epsilon}\right)$, a trajectory that joins $\operatorname{Id}_{x_{0}}$ at $\epsilon=0$ to $\psi$ 
at $\epsilon=1$. This tangent vector is:

$$
\Delta_{G}(\psi)=\left(\frac{\mathrm{d}}{\mathrm{d} \epsilon}\right)_{0} \sigma_{\psi}(\epsilon)=\left(\mathrm{d}_{0_{x}} \tau\right)\left(A_{\psi}\right)+\left(\mathrm{d}_{\left(x_{0}, x_{0}\right)} \mathcal{H}\right)\left(\Delta^{X}\left(x_{0}, x_{1}\right)\right)=\tau^{-1}\left(\mathcal{V}^{s}(\psi)\right)+\left\langle\chi_{s(\psi)}^{\mathcal{H}}, \Delta^{X}(s(\psi), t(\psi))\right\rangle
$$

where we use that $\tau\left(0_{x}\right)=\operatorname{Id}_{x}=\mathcal{H}(x, x),\left(\mathrm{d}_{0_{x}} \tau\right)\left(A_{\psi}\right)=A_{\psi}$ for the retraction map $\tau,(d / d \epsilon)_{0} x_{\epsilon}=\Delta^{X}\left(x_{0}, x_{1}\right)$, and the definition of the connection $\chi^{\mathcal{H}}$ associated to a parallelism $\mathcal{H}$.

First of all, to prove that $\Delta_{G}$ factors by $\Delta^{X}: U_{d} \rightarrow T X$ it suffices to observe that $\tau^{-1}\left(\mathcal{V}^{s}(\psi)\right) \in \operatorname{Ad} P \subset T_{G} P$ projects as 0 by $\overline{\mathrm{d} \pi}: T_{G} P \rightarrow T X$ and that $\left\langle\chi_{s(\psi)}^{\mathcal{H}}, \Delta^{X}(s(\psi), t(\psi))\right\rangle \in T_{G} P$ projects as $\Delta^{X}(s(\psi), t(\psi)) \in T X$ (because $\chi$ is a connection). Our mapping $\Delta_{G}$ is projectable as $\Delta^{X}$.

As $\Delta^{X}(x, x)=0$ and $\mathcal{V}^{s}$ is the identity on Gau $P$, we observe that $\Delta_{G}$ on Gau $P_{x} \subset$ End $P$ is just the mapping $\tau^{-1}$. As $\tau$ was a retraction map, whose differential at the identity was the identity on the adjoint bundle, we conclude that the differential of $\Delta_{G}$ on $V^{(s, t)}$ End $P \subset V^{s}$ End $P$ is the identity map. We still have to study this differential on $V^{s}$ End $P$.

For any choice $x_{0}, x_{1} \in X$, consider $x_{\epsilon}=\alpha_{x_{0} x_{1}}(\epsilon)$ (as in Theorem 2.2). Consider the curve $\psi_{\epsilon}=\mathcal{H}\left(x_{0}, x_{\epsilon}\right)$ on End $P$, that for $\epsilon=0$ has a tangent vector $\left(\mathrm{d}_{\left(x_{0}, x_{0}\right)} \mathcal{H}\right)\left(\Delta^{X}\left(x_{0}, x_{1}\right)\right)=\left\langle\chi_{x_{0}}^{\mathcal{H}}, \Delta^{X}\left(x_{0}, x_{1}\right)\right\rangle$. Its image by $\Delta_{G}$ is a curve on $\left(T_{G} P\right)_{x_{0}}$ defined as $\Delta_{G}\left(\psi_{\epsilon}\right)=\left\langle\chi_{x_{0}}^{\mathcal{H}}, \Delta^{X}\left(x_{0}, x_{\epsilon}\right)\right\rangle$ (because $\psi_{\epsilon}$ has a trivial vertical component). Taking into account that $\Delta^{X}\left(x_{0}, x_{\epsilon}\right)=\epsilon \Delta^{X}\left(x_{0}, x_{1}\right)$, the tangent vector of this curve $\Delta_{G}\left(\psi_{\epsilon}\right) \subset\left(T_{G} P\right)_{x_{0}}$ at $\epsilon=0$ is identified with $\left\langle\chi_{x_{0}}^{\mathcal{H}}, \Delta^{X}\left(x_{0}, x_{1}\right)\right\rangle$.

We conclude that $\mathrm{d}_{\mathrm{Id}} \Delta_{G}$ is the identity map on all elements with the form $\left\langle\chi_{x_{0}}^{\mathcal{H}}, \Delta^{X}\left(x_{0}, x_{1}\right)\right\rangle$, where $x_{1}$ is arbitrary on an open neighbourhood of $x_{0}$. As $\Delta^{X}(x, \cdot)$ is a local diffeomorphism from a neighborhood of $x \in X$ to a neighbourhood of $0_{x} \in T_{x} X$, we conclude that $\mathrm{d}_{\operatorname{Id}} \Delta_{G}$ is the identity on all elements of $\operatorname{Im} \chi_{x}^{\mathcal{H}} \subset\left(T_{G} P\right)_{x} \simeq$ $V_{I d_{x}}^{s}$ End $P$.

As we know, for any principal connection $\chi$ the image $\operatorname{Im} \chi_{x} \subset\left(T_{G} P\right)_{x}$ is a complementary subspace to $\operatorname{Ad} P_{x}$, and therefore we conclude that $\mathrm{d}_{\operatorname{Id}_{x}} \Delta_{G}$ is the identity map on the whole space $V_{\operatorname{Id}_{x}}^{s}$ End $P$. As $\Delta_{G}$ is a local diffeomorphism on some neighborhood of the identity section, it will be a diffeomorphism when restricted to some smaller neighborhood. On this neighborhood, the RFD operator $\Delta_{G}$ is then a faithful RFD operator, projectable to $\Delta^{X}$, and whose restriction to Gau $P$ is an inverse of the retraction map $\tau$.

\section{Discretization and reduction of Lagrangian densities}

Consider a volume form $\operatorname{vol}_{X}$ on a manifold $X$ and a smooth Lagrangian function $\mathcal{L} \in \mathcal{C}^{\infty}(J P)$, for some principal $G$-bundle $\pi: P \rightarrow X$.

In the case that the Lagrangian is $H$-invariant for some closed subgroup $H \subseteq G$, we know that the corresponding trivialized Lagrangian function $\ell(p, \chi): P \times_{X} \mathrm{CP} \rightarrow \mathbb{R}$ (Definition 4.12) is invariant for $H$ acting on the first component, and can be expressed as a reduced Lagrangian function $\ell(q, \chi): H \operatorname{Str} \times{ }_{X} \mathrm{CP} \rightarrow \mathbb{R}$, where $H \operatorname{Str}=$ $P / H$ is the bundle of $H$-structures and CP the bundle of principal connections. Moreover, following Corollary 4.2 , if $\mathcal{L}$ is $\phi$-invariant for some gauge transformation $\phi: P \rightarrow P$, the associated $H$-reduced Lagrangian is also $\phi$-invariant.

On the other hand, in the case that we consider a faithful projectable FD operator on $P$, the corresponding discrete volume function $\widetilde{\text { vol }}: \widetilde{X} \rightarrow \mathbb{R}^{+}$and FJ operator $J_{X}^{P}: \widetilde{J P} \rightarrow \pi_{0}^{*} J P$ allow to use $\mathcal{L}_{\text {vol }_{X}}$ to generate the corresponding discretized Lagrangian $\mathcal{L}_{d}: \widetilde{J P} \rightarrow \mathbb{R}$ (Definition 5.12), determined on the regular domain $\widetilde{J P} \subset$ $P^{\times n}$. This function only depends on the Lagrangian density $\mathcal{L} \mathrm{vol}_{X}$ and not on the particular decomposition as a volume form, on the base manifold multiplied with a function on the jet bundle. Moreover, following Proposition 5.3, if $\mathcal{L}$ is $H$-invariant then the associated discrete Lagrangian $\mathcal{L}_{d}$ is also $H$-invariant.

Is there a coherent way to $H$-reduce discrete Lagrangian functions $\mathcal{L}_{d}$, that are $H$-invariant and to discretize $H$ reduced Lagrangian densities $\ell \operatorname{vol}_{X}$ ? In this section, we shall introduce the space where reduced discrete theories have a sense and will explore the interplay of the reduction and discretization procedures for Lagrangian densities $\mathcal{L} \mathrm{Vol}_{X}$ that are $H$-invariant, when we employ covariant faithful FD operators (that is, faithful projectable RFD operators). 
Proposition 8.1

Consider any principal $G$-bundle $\pi: P \rightarrow X$. There exists a natural identification of the pull-back $\pi^{*}(\text { End } P)^{\times}{ }_{s} n-1$ of the bundle $s$ : (End $P)^{\times}{ }_{s} n-1 \rightarrow X$ by $\pi: P \rightarrow X$ with the bundle $\pi_{0}: P^{\times n} \rightarrow P$. Using this identification, the projector $\pi^{\times n}: P^{\times n} \rightarrow X^{\times n}$ gets identified with $\left(s, t^{\times n-1}\right): P \times_{(\pi, s)}(\text { End } P)^{\times_{s} n-1} \rightarrow X^{\times n}$. Moreover, the action $\lambda_{g}^{\times n}$ on $P^{\times n}$ gets identified with $\lambda_{g} \times \operatorname{Id}$ on $P \times{ }_{(\pi, s)}(\text { End } P)^{\times}{ }_{s} n-1$, and for any gauge transformation $\phi: P \rightarrow P$ the action $\phi^{\times n}$ gets identified with $\phi \times \phi_{\text {End }}^{\times n-1}$.

Proof

It suffices to apply Proposition 7.1. The identification is then given by $\left(p_{0}, \ldots, p_{n}\right) \in P^{\times n} \mapsto$ $\left(p_{0}, p_{0}^{-1} p_{1}, \ldots, p_{0}^{-1} p_{n}\right) \in P \times_{(\pi, s)}(\text { End } P)^{\times_{s} n-1}$ and its inverse $\left(p_{0}, \psi_{1}, \ldots, \psi_{n}\right) \in P \times_{(\pi, s)}(\text { End } P)^{\times_{s} n-1} \mapsto$ $\left(p_{0}, p_{0} \psi_{1}, \ldots, p_{0} \psi_{n}\right) \in P^{\times n}$.

Corollary 8.1

There exists a natural identification of the pull-back $\pi_{H \operatorname{Str}}^{*}(\text { End } P)^{{ }^{\times}{ }_{s} n-1}$ of the bundle $s:(\text { End } P)^{\times}{ }_{s} n-1 \rightarrow X$ by $\pi_{H \text { Str }}: H$ Str $=P / H \rightarrow X$ with the bundle $\pi_{0}: P^{\times n} / H \rightarrow P / H=H$ Str. For any gauge transformation $\phi: P \rightarrow P$, the action $\phi^{\times n}$ factors in the quotient $P^{\times n} / H \simeq H \operatorname{Str} \times{ }_{X}(\text { End } P)^{\times{ }_{s} n-1}$ as $a c_{\phi} \times \phi_{\text {End }}^{\times n-1}$, where $a c_{\phi}: H p \in P / H \mapsto H(p \phi) \in P / H$.

Recall that the FJ operator $J_{X}^{P}$ associated to a faithfull covariant projectable FD operator (equivalently, a faithful projectable RFD operator) is a covariant injective local diffeomorphism $\widetilde{J P} \rightarrow \pi_{0}^{*}(J P)$ and determines a mapping $\widetilde{J P} / G \rightarrow \pi_{0}^{*}(J P / G)$ on the quotient manifolds. Using the identifications $J P \simeq P \times{ }_{X} \mathrm{CP}$ and $P^{\times n} \simeq$ $P \times_{X}(\text { End } P)^{\times_{s} n-1}$, where the group $G$ acts only on the leading $P$-component, we get a more convenient expression for the FJ operator $J_{X}^{P}$, that we might express in terms of the RFD operator.

\section{Definition 8.1}

For a fixed projectable RFD operator $\Delta_{G}: U_{\bar{e}} \rightarrow T_{G} P$, we denote by $\tilde{\pi}_{\mathrm{CP}}=\widetilde{\mathrm{CP}} \rightarrow \widetilde{X}$ and call discrete connection bundle associated to the principal $G$-bundle $\pi: P \rightarrow X$, the open sub-bundle of the bundle $\left(s, t^{\times n-1}\right)$ : (End $\left.P\right)^{\times{ }_{s} n-1} \rightarrow X^{\times n}$ given by elements $\left(\psi_{1}, \ldots, \psi_{n}\right)$ such that, $\Delta_{G}\left(\psi_{i}\right)$ is defined and whose projection by $\left(s, t^{\times n-1}\right)$ is a point $\left(x_{0}, x_{1}, \ldots, x_{n}\right)$ in the set $\widetilde{X} \subset X^{\times n}$ of regular points. That is, $\widetilde{\mathrm{CP}}=$ $\left(U_{\bar{e}}\right)^{{ }_{s} n-1} \cap\left(s, t^{\times n-1}\right)^{-1} \widetilde{X}$.

One element in $\widetilde{\mathrm{CP}}$ is a regular point $\left(x_{0}, \ldots, x_{n}\right) \in \widetilde{X} \subset X^{\times n}$ together with a sequence of fiber-to-fiber endomorphisms $\psi_{i} \in$ End $P(i=1 \ldots n)$ in the domain of $\Delta_{G}$, with $s\left(\psi_{i}\right)=x_{0}, t\left(\psi_{i}\right)=x_{i} \forall i=1, \ldots, n$. Considering now the restriction of the previous identification to the open sub-bundle $\widetilde{J P} \subset P^{\times n}$ (see Definition 5.5), we have:

\section{Corollary 8.2}

There exists a natural diffeomorphism of the bundle $\pi^{*} \widetilde{\mathrm{CP}}=P \times_{X} \widetilde{\mathrm{CP}} \rightarrow \pi^{*} \widetilde{X}$ with the bundle $\widetilde{J P} \rightarrow \pi_{0}^{*} P$ (where $\left.\pi_{0}: \widetilde{X} \rightarrow X, \pi: P \rightarrow X\right)$. This identification transforms the action $\lambda_{g} \times \operatorname{Id}$ on $\pi^{*} \widetilde{\mathrm{CP}}=P \times{ }_{X} \widetilde{\mathrm{CP}}$ into the action $\lambda_{g}^{\times n}$ on $\widetilde{J P} \subset P^{\times n}$. It also identifies the action $\phi \times \mathrm{Conj}_{\phi}^{\times n-1}$ with $\phi^{\times n}$, for any gauge transformation $\phi$.

In the quotient, by the action of any closed subgroup $H \subseteq G$, this induces a natural diffeomorphism of the bundle $\pi_{H \text { Str }}^{*} \widetilde{C P}=H \operatorname{Str} \times{ }_{X} \widetilde{\mathrm{CP}} \rightarrow \widetilde{X}$ with the bundle $\widetilde{J P} / H \rightarrow \widetilde{X}$. The action $\phi^{\times n}$ of any gauge transformation $\phi$ on $\widetilde{J P}$ factors in the quotient by $H$ as $a c_{\phi} \times \operatorname{Conj}_{\phi}^{\times n-1}$, using the identification $\widetilde{J P} / H \simeq H \operatorname{Str} \times{ }_{X} \widetilde{C P}$.

In the same manner as projectable FD operators induce corresponding FJ operators, also projectable RFD operators induce reduced Forward Jacobi operators.

\section{Proposition 8.2}

Consider the projectable covariant FD operator $\Delta$ on $P$, determined by some projectable RFD operator $\Delta_{G}: U_{\bar{e}} \rightarrow$ $T_{G} P$ on a principal $G$-bundle $\pi: P \rightarrow X$. The $n$-Jacobian immersion $J^{P}: P^{\times n} \rightarrow \pi_{0}^{*}\left(T P \otimes \mathbb{R}^{n}\right)$ and FJ operator $J_{X}^{P}: \widetilde{J P} \rightarrow \pi_{0}^{*}(J P)$ associated to $\Delta$ (given by Lemma 5.3 and Definition 5.9) factor by the diagonal action of $G$ on $P^{\times n}$, the tangent action on $T P \otimes \mathbb{R}^{n}$ and the 1-jet action on $J P$. The corresponding projected morphisms (reduced 
$n$-Jacobian map and reduced FJ operator) are:

$$
\begin{aligned}
& R J^{P}:\left(\psi_{1}, \ldots, \psi_{n}\right) \in(\text { End } P)^{\times_{s} n-1} \mapsto \sum \Delta_{G}\left(\psi_{i}\right) \otimes e_{i} \in \pi_{0}^{*}\left(T_{G} P \otimes \mathbb{R}^{n}\right) \\
& J_{\mathrm{CP}}=c_{\mathbb{R}^{n}}\left(R J^{P} \otimes J_{X}^{-1}\right): \widetilde{\mathrm{CP}} \rightarrow \pi_{0}^{*}(\mathrm{CP}) \subset \pi_{0}^{*}\left(T_{G} P \otimes T^{*} X\right)
\end{aligned}
$$

Proof

To prove that these operators factor by the $G$-action, it suffices to apply the covariance properties stated in Lemma 5.2 and Theorem 5.1, for each of the bundle automorphisms $\lambda_{g}: P \rightarrow P$. The particular expressions for the induced morphism $R J^{P}$ on the quotient manifolds is a direct consequence of the definition for $\Delta_{G}$. The particular expression for $J_{\mathrm{CP}}$ is a consequence of the definition of $J_{X}^{P}$, using $c_{\mathbb{R}^{n}}\left(J^{P} \otimes J_{X}^{-1}\right)$ and the factorization of $J^{P}$ as $R J^{P}$.

\section{Definition 8.2}

For a projectable, faithful RFD operator $\Delta_{G}: U_{\bar{e}} \rightarrow T_{G} P$, we call Reduced $n$-Jacobian immersion $R J^{P}:(\text { End } P)^{\times}{ }_{s}^{n-1} \rightarrow \pi_{0}^{*}\left(T_{G} P \otimes \mathbb{R}^{n}\right)$ and Reduced Forward Jacobi $(\mathrm{RFJ})$ operator $J_{\mathrm{CP}}: \widetilde{\mathrm{CP}} \rightarrow \pi_{0}^{*}(\mathrm{CP})$, respectively, the mappings given in (17).

Our previous results (Theorem 5.1, Corollary 8.2 and Proposition 8.2) lead to the first relevant property of the RFJ operator:

Theorem 8.3

The RFJ operator $J_{\mathrm{CP}}: \widetilde{\mathrm{CP}} \rightarrow \pi_{0}^{*}(\mathrm{CP})$ is an injective local diffeomorphism of $\widetilde{X}$-bundles. Moreover, if $\Delta_{G}$ is covariant for some gauge transformation $\phi: P \rightarrow P$, there holds:

$$
J_{\mathrm{CP}} \circ \operatorname{Conj}_{\phi}^{\times n-1}=(\mathrm{c} \phi) \circ J_{\mathrm{CP}}
$$

where for any gauge transformation $\phi$, we denote $\operatorname{Conj}_{\phi} \psi=\phi_{t(\psi)} \circ \psi \circ \phi_{s(\psi)}^{-1}: P_{s(\psi)} \rightarrow P_{t(\psi)}$, for each $\psi \in$ End $P$, and $\mathrm{c} \phi: \mathrm{CP} \rightarrow \mathrm{CP}$ is the morphism induced by $j \phi: J P \rightarrow J P$ on the quotient $\mathrm{CP}=J P / G$.

If $P \rightarrow X$ is a principal $G$-bundle, $H \subseteq G$ a closed subgroup, and $\Delta_{G}$ a faithful projectable RFD operator, all definitions involved lead to the following commutative diagram, where all morpisms are covariant for the natural action of any gauge transformation $\phi: P \rightarrow P$, on the corresponding spaces:

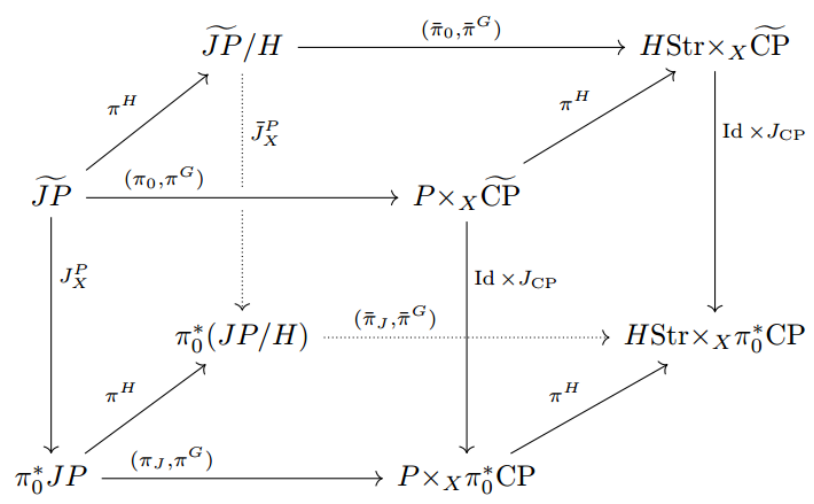

Figure 2. Commutative diagram.

In this diagram the upper side refers to discrete theory, lower side to smooth theory, left hand side to non trivialized (jet bundle) formulation, right hand side to trivialized (principal connection bundle) formulation, front side to unreduced formalism, back side to $H$-reduced formalism. Morphisms going downwards are Forward-Jacobi operators relating smooth and discrete formulations. Morphisms going rightwards are trivialization isomorphisms 
relating jets with principal connections. Finally, morphisms going backwards are quotient morphisms by the $H$ action, relating any formalism with its $H$-reduced version.

Moreover, in a similar way as in Theorem 5.2, in the case of faithful projectable RFD operators, the reduced forward Jacobi operator has the expected behaviour when one considers points that coalesce to a certain diagonal point $\left(x_{0}, \ldots, x_{0}\right)$. To be precise:

\section{Theorem 8.4}

Let $\Delta_{G}: U_{\bar{e}} \rightarrow T_{G} P$ be a faithful projectable RFD operator for the principal $G$-bundle $\pi: P \rightarrow X$ and $J_{\mathrm{CP}}: \widetilde{\mathrm{CP}} \rightarrow$ $\pi_{0}^{*}(\mathrm{CP})$ the associated RFJ operator.

Consider any smooth trajectory $\tilde{x}(\epsilon)=\left(x_{0}, x_{1}(\epsilon), \ldots, x_{n}(\epsilon)\right)_{0<\epsilon<\epsilon_{\max }} \subset \widetilde{X}$ coalescing to a diagonal point (see Definition 5.10) and contained in the regular domain $\tilde{X} \subset X^{\times n}$. For each parallelism $\mathcal{H}: U_{d} \rightarrow$ End $P$, there exists $\epsilon_{\max }>0$ so that, the induced smooth trajectory $\tilde{\psi}(\epsilon)=\left(\psi_{i}(\epsilon)\right)_{i=1 \ldots n} \in \widetilde{\mathrm{CP}}_{x_{0}}$ defined by $\psi_{i}(\epsilon)=\mathcal{H}\left(x_{0}, x_{i}(\epsilon)\right)$ belongs to the regular domain $\widetilde{\mathrm{CP}}$, for $0<\epsilon<\epsilon_{\max }$ and is transformed by the RFJ operator into a trajectory $J_{\mathrm{CP}}(\epsilon) \in \mathrm{CP}_{x_{0}}$, whose limit value for $\epsilon \rightarrow 0^{+}$(if it exists) coincides with the value at $x_{0}$ of the connection $\chi^{\mathcal{H}}$ associated to the parallelism $\mathcal{H}$.

\section{Proof}

As $\lim x_{i}(\epsilon)=x_{0}$, and $U_{d}$ is an open neighbourhood of the diagonal, we get $\epsilon_{\max }>0$ such that $\left(x_{0}, x_{i}(\epsilon)\right) \in U_{d}$ for $\epsilon<\epsilon_{\max }$. Moreover, there holds $\lim \mathcal{H}\left(x_{0}, x_{i}(\epsilon)\right)=\operatorname{Id}_{x_{0}}$ and therefore as $U_{\bar{e}}$ is an open neighbourhood of the unitary section, for a possibly smaller $\epsilon_{\max }$ we can assure that $\psi_{i}(\epsilon)=\mathcal{H}\left(x_{0}, x_{i}(\epsilon)\right) \in U_{\bar{e}}$ for $0<\epsilon<\epsilon_{\max }$. As the trajectory $\tilde{x}(\epsilon)=\left(x_{0}, x_{1}(\epsilon), \ldots, x_{n}(\epsilon)\right)$ was contained in $\tilde{X}$ for $\epsilon \neq 0$, we conclude that $\tilde{\psi}(\epsilon)$ is contained in the regular domain $\widetilde{\mathrm{CP}}$ for $0<\epsilon<\epsilon_{\max }$.

Both $J^{X}$ and $R J^{P}$ are smooth mappings and transform smooth curves $\tilde{x}(\epsilon)$ and $\tilde{\psi}(\epsilon)$ into smooth curves $J^{X}(\epsilon)$, $R J^{P}(\epsilon)$.

By definition, we have:

$$
\begin{aligned}
& R J^{P}(\tilde{\psi})=\sum \Delta_{G}\left(\psi_{i}\right) \otimes e_{i} \Rightarrow\left\langle R J^{P}(\tilde{\psi}), e_{i}\right\rangle=\Delta_{G}\left(\psi_{i}\right) \quad\left(\tilde{\psi}=\left(\psi_{i}\right)_{i=1 \ldots n}\right) \\
& \psi_{i}(0)=\operatorname{Id}_{x_{0}}, s\left(\psi_{i}(\epsilon)\right)=x_{0} \Rightarrow \dot{\psi}_{i}(0) \in V_{\operatorname{Id}_{x_{0}}}^{s} \text { End } P
\end{aligned}
$$

Taking now into account that $\Delta_{G}$ is faithful and projectable to $\Delta^{X}$ we get:

$$
\begin{gathered}
\dot{\psi}_{i}(0)=\left(\mathrm{d} \Delta_{G}\right)_{\operatorname{Id}_{x_{0}}}\left(\dot{\psi}_{i}(0)\right)=\lim _{\epsilon \rightarrow 0^{+}} \frac{\Delta_{G}\left(\psi_{i}(\epsilon)\right)-\Delta_{G}\left(\operatorname{Id}_{x_{0}}\right)}{\epsilon}=\lim _{\epsilon \rightarrow 0^{+}} \frac{\left\langle R J^{P}(\epsilon), e_{i}\right\rangle-0}{\epsilon} \\
\dot{x}_{i}(0)=\left(\mathrm{d} \Delta^{X}\right)_{\left(x_{0}, x_{0}\right)}\left(\dot{x}_{i}(0)\right)=\lim _{\epsilon \rightarrow 0^{+}} \frac{\Delta^{X}\left(x_{0}, x_{i}(\epsilon)\right)-\Delta^{X}\left(x_{0}, x_{0}\right)}{\epsilon}=\lim _{\epsilon \rightarrow 0^{+}} \frac{\left\langle J^{X}(\epsilon), e_{i}\right\rangle-0}{\epsilon}
\end{gathered}
$$

where $\dot{x}_{i}(0) \in T_{x_{0}} X, \dot{\psi}_{i}(0) \in V_{\mathrm{Id}_{x_{0}}}^{s}$ End $P$ stand for the tangent vectors at $\epsilon=0$ of the given curve $x_{i}(\epsilon)$ and its image $\psi_{i}(\epsilon)$.

Hence, if there exists a limit $J_{0}$ for $J_{\mathrm{CP}}(\epsilon) \in \mathrm{CP}_{x_{0}} \subset \mathrm{Ad} P_{x_{0}} \otimes T_{x_{0}}^{*} X$ as $\epsilon$ tends to zero, we have:

$$
\left\langle J_{0}, \dot{x}_{i}(0)\right\rangle=\left\langle\lim _{\epsilon \rightarrow 0^{+}} J_{\mathrm{CP}}(\epsilon), \lim _{\epsilon \rightarrow 0^{+}} \frac{\left\langle J^{X}(\epsilon), e_{i}\right\rangle}{\epsilon}\right\rangle=\lim _{\epsilon \rightarrow 0^{+}} \frac{\left\langle J_{\mathrm{CP}}(\epsilon),\left\langle J^{X}(\epsilon), e_{i}\right\rangle\right\rangle}{\epsilon}=\lim _{\epsilon \rightarrow 0^{+}} \frac{\left\langle R J^{P}(\epsilon), e_{i}\right\rangle}{\epsilon}=\dot{\psi}_{i}(0)
$$

On the other hand, by definition:

$$
\chi_{x}^{\mathcal{H}}=\mathrm{d}_{(x, x)} \mathcal{H} \in \mathrm{CP}_{x}, \quad \mathcal{H}\left(x_{0}, x_{i}(\epsilon)\right)=\psi_{i}(\epsilon) \Rightarrow\left\langle\chi_{x_{0}}^{\mathcal{H}}, \dot{x}_{i}(0)\right\rangle=\left\langle\mathrm{d}_{\left(x_{0}, x_{0}\right)} \mathcal{H}, \dot{x}_{i}(0)\right\rangle=\dot{\psi}_{i}(0)
$$

Therefore both $J_{0}$ and $\chi_{x_{0}}^{\mathcal{H}}$ coincide on a certain basis of $T_{x_{0}} X$, which proves that they coincide as linear mappings from $T_{x_{0}} X$ to Ad $P_{x_{0}}$. 
This result parallels the result obtained in Theorem 5.2 for non-reduced FD operators. In the same manner as a section $y \in \Gamma(Y)$ generates (fixing $\tilde{x} \in \widetilde{X}$ ) two objects $\widetilde{y} \in Y^{\times n}$ and $j_{x_{0}}$ y, being the first one a discrete approximation (through $J_{X}^{Y}$ ) of the second one, also a parallelism $\mathcal{H} \in \Gamma($ End $P$ ) generates two objects $\tilde{\psi} \in \widetilde{\mathrm{CP}}$ and $\chi_{x_{0}}^{\mathcal{H}} \in \mathrm{CP}_{x_{0}}$, where the first one is a discrete approximation (through $J_{\mathrm{CP}}$ ) of the second one. These approximations are the identity in the limit case, when regular points $\tilde{x}$ coalesce to a diagonal point. Moreover, it justifies our choice of $\widetilde{\mathrm{CP}} \subset(\text { End } P)^{\times{ }_{s} n-1}$ as discrete connection bundle, rather than simply $(P \times P) / G=$ End $P$. However, for the case of mechanics $X=\mathbb{R}, n=1$, and for principal $G$-bundles on $\mathbb{R}$, both notions coincide.

Finally, we are in situation to create $H$-reduced discrete Lagrangians. There are two possibilities, namely reducing a given discrete Lagrangian function $\mathcal{L}_{d}: \widetilde{J P} \rightarrow \mathbb{R}$ that is $H$-invariant, or discretizing a given $H$-reduced Lagrangian density $\ell \cdot \operatorname{vol}_{X}$, where $\ell \in \mathcal{C}^{\infty}\left(H \operatorname{Str} \times_{X} \widetilde{\mathrm{CP}}\right)$.

\section{Definition 8.3}

We call trivialized discrete Lagrangian associated to a discrete Lagrangian $\mathcal{L}_{d}\left(p_{0}, \ldots, p_{n}\right): \widetilde{J P} \rightarrow \mathbb{R}$, the function $\ell_{d}\left(p, \psi_{1}, \ldots, \psi_{n}\right): P \times_{X} \widetilde{\mathrm{CP}} \rightarrow \mathbb{R}$ determined by $\mathcal{L}_{d}$, using the identification $\widetilde{J P} \simeq P \times_{X} \widetilde{\mathrm{CP}}$ given in Corollary 8.2.

We say that a given discrete Lagrangian function $\mathcal{L}_{d}: \widetilde{J P} \rightarrow \mathbb{R}$ is $H$-invariant if there holds $\mathcal{L}_{d} \circ \lambda_{h}^{\times n}=\mathcal{L}_{d}$, for each $h \in H$. In this case, the associated trivialized discrete Lagrangian $\ell_{d}$ satisfies $\ell_{d} \circ\left(\lambda_{h} \times \mathrm{Id}\right)=\ell_{d}$ and factors by a function $\ell_{d}\left(q, \psi_{1}, \ldots, \psi_{n}\right): H \operatorname{Str} \times{ }_{X} \widetilde{\mathrm{CP}} \rightarrow \mathbb{R}$ that we call $H$-reduced discrete Lagrangian associated to $\mathcal{L}_{d}$.

As in the case of smooth Lagrangian densities, for the case $H=\{e\}$, the $H$-reduced discrete Lagrangian is the trivialized discrete Lagrangian associated to $\mathcal{L}_{d}$. Also for the case $H=G$, the $H$-reduced discrete Lagrangian shall be called simply the reduced discrete Lagrangian, a function $\ell_{d}: \widetilde{\mathrm{CP}} \rightarrow \mathbb{R}$.

\section{Definition 8.4}

For any $H$-reduced Lagrangian density $\ell \operatorname{vol}_{X}$ determined by a volume function $\operatorname{vol}_{X}$ and a smooth function $\ell: H \operatorname{Str} \times{ }_{X} \mathrm{CP} \rightarrow \mathbb{R}$, we call discretized $H$-reduced Lagrangian, $\ell_{d}: H \operatorname{Str} \times{ }_{X} \widetilde{\mathrm{CP}} \rightarrow \mathbb{R}$, the function

$$
\ell_{d}=\left(\ell \circ\left(\operatorname{Id} \times J_{\mathrm{CP}}\right)\right) \cdot\left(\widetilde{\operatorname{vol}} \circ\left(s, t^{\times n-1}\right)\right): H \operatorname{Str} \times{ }_{X} \widetilde{\mathrm{CP}} \rightarrow \mathbb{R}
$$

where $\mathrm{Id} \times J_{\mathrm{CP}}: H \operatorname{Str} \times{ }_{X} \widetilde{\mathrm{CP}} \rightarrow \pi_{0}^{*}\left(H \operatorname{Str} \times_{X} \mathrm{CP}\right)$ is associated to $\Delta_{G}, \widetilde{\operatorname{vol}}$ is defined in (9) and $\left(s, t^{\times n-1}\right): \widetilde{\mathrm{CP}} \rightarrow$ $\widetilde{X}$ is the restriction of $\left(s, t^{\times n-1}\right)$ : (End $\left.P\right)^{\times_{s} n-1} \rightarrow X^{\times n}$ to the set of regular points.

\section{Remark 8.1}

In the same way as for the non-reduced formulation, from the definition of $J_{\mathrm{CP}}$ we observe that for any smooth function on the base manifold, $f \in \mathcal{C}^{\infty}(X)$, any $H$-reduced Lagrangian function $\ell \in \mathcal{C}^{\infty}\left(H \operatorname{Str} \times{ }_{X} \mathrm{CP}\right)$, and any volume form $\operatorname{vol}_{X} \in \Omega^{n}(X)$ there holds

$$
(f \cdot \ell) \circ\left(\operatorname{Id} \times J_{\mathrm{CP}}\right)=\left(f \circ \pi_{0}\right) \cdot\left(\ell \circ\left(\operatorname{Id} \times J_{\mathrm{CP}}\right)\right), \quad \widetilde{f \cdot \operatorname{vol}}=\left(f \circ \pi_{0}\right) \cdot \widetilde{\mathrm{vol}}
$$

As a consequence, the discrete $H$-reduced Lagrangian $\ell_{d}$ associated to a density $\ell \operatorname{vol}_{X}$ only depends on the particular RFD operator $\Delta_{G}$ and on the reduced Lagrangian density $\ell \mathrm{vol}_{X}$, but not on the particular decomposition as a volume form $\operatorname{vol}_{X} \in \Omega^{n}(X)$ and a smooth function $\ell \in \mathcal{C}^{\infty}(\mathrm{CP})$.

\section{Remark 8.2}

If $\mathcal{H} \in \Gamma($ End $P)$ is a locally defined parallelism, $q \in \Gamma(H$ Str $)$ and if $K \subset X$ is a simplicial domain with vertices $x_{0}, x_{1}, \ldots, x_{n} \in X$, ordered so that $\Delta^{X}\left(x_{0}, x_{i}\right)$ are a positively oriented basis on $T_{x_{0}} X$, taking into account Theorem 8.4 and considering a quadrature rule for $\int_{K} \ell \operatorname{vol}_{X}$ with a single node $x_{0}$ (resembling the arguments that led to Definition 5.4), we shall consider $\ell_{d}\left(q_{x_{0}}, \mathcal{H}\left(x_{0}, x_{1}\right), \ldots, \mathcal{H}\left(x_{0}, x_{n}\right)\right) \in \mathbb{R}$ to be a good approximation of the action functional associated to $\ell \mathrm{vol}_{X}$, on the domain $K$ :

$$
\int_{x \in K} \ell\left(q_{x}, \chi_{x}^{\mathcal{H}}\right) \operatorname{vol}_{X} \simeq \ell_{d}\left(q_{x_{0}}, \mathcal{H}\left(x_{0}, x_{1}\right), \ldots, \mathcal{H}\left(x_{0}, x_{n}\right)\right)
$$

Our discretization mechanisms are, furthermore, covariant, and commute with the reduction mechanisms: 
Theorem 8.5

Let $\pi: P \rightarrow X$ be a principal bundle and $\Delta_{G}$ a faithful projectable RFD operator. Consider the bundles $\widetilde{J P}$ and $\widetilde{C P}$ of discrete jets and connections, and the associated FJ and RFJ operators. Consider any gauge transformation $\phi: P \rightarrow P$. If $\Delta_{G}$ is covariant for $\phi$ there holds that,

1. Any given $H$-invariant discrete Lagrangian function $\mathcal{L}_{d}: \widetilde{J P} \rightarrow \mathbb{R}$ that is $\phi$-invariant, induces a $\phi$-invariant $H$-reduced discrete Lagrangian function $\ell_{d}: H \operatorname{Str} \times{ }_{X} \widetilde{\mathrm{CP}} \rightarrow \mathbb{R}$.

2. Any given $H$-reduced Lagrangian density $\ell \operatorname{vol}_{X}$ determined by some $\phi$-invariant function $\ell \in$ $\mathcal{C}^{\infty}\left(H \operatorname{Str} \times_{X} \mathrm{CP}\right)$ and volume form $\operatorname{vol}_{X} \in \Omega^{n}(X)$, induces an $H$-reduced discrete Lagrangian function $\ell_{d}: H \operatorname{Str} \times_{X} \widetilde{\mathrm{CP}} \rightarrow \mathbb{R}$ that is $\phi$-invariant.

3. If $\mathcal{L}: J P \rightarrow \mathbb{R}$ is an $H$-invariant function and $\operatorname{vol}_{X} \in \Omega^{n}(X)$ a volume form, then the associated $H$-reduced Lagrangian denstity $\ell \mathrm{vol}_{X}$ and the associated discrete Lagrangian function $\mathcal{L}_{d}$ generate, by discretization in the first case, and by $H$-reduction in the second case, the same $H$-reduced discrete Lagrangian function $\ell_{d}: H \operatorname{Str} \times_{X} \widetilde{\mathrm{CP}} \rightarrow \mathbb{R}$.

Proof

It is a direct consequence of the fact that the Figure 2 is commutative, and all its morphisms covariate for the natural action of a gauge transformation $\phi: P \rightarrow P$ on the corresponding bundles.

\section{Concluding remarks and future work}

In the same manner as numerical algorithms have been extended to manifolds using FD operators (or retraction maps) $[1,17,20,38]$, in the category of fibred manifolds numerical algorithms dealing at the same time with objects and its projections to a quotient space may benefit from the idea of projectable FD operators. A particular situation would be that of numerical algorithms dealing with mappings between two manifolds or more generally, with tangent fields, moving frames on a manifold or similar geometrical objects.

In this category of fibred manifolds, leaving aside the simplest case of trivial bundles, our work focuses in the study of general bundles where one has a Lie group and a free, proper action, transitive on each fiber. With this we don't pretend to restrict our tools to the specific situation of principal bundles, but also to include the next interesting situation: that of a general group acting freely by $X$-bundle automorphisms on a bundle $\pi: Y \rightarrow X$. This bundle is obtained as the composition of a $G$-principal bundle $\pi^{G}: Y \rightarrow Y / G$ and the bundle $\bar{\pi}: Y / G \rightarrow X$. Covariant projectable difference operators and a covariant discretization of Lagrangian densities in this case could be achieved using the general theory in section 5 using any $\bar{\pi}$-projectable faithful FD operator $\Delta$ on the bundle $\bar{\pi}$, and its extension by a choice of faithful RFD operator $\Delta_{G}$ for the principal $G$-bundle $\pi^{G}$, projecting to $\Delta$. In this sense the applications of the work presented here do not restrict to principal bundles, but to general bundles with a free, proper Lie group action. In this area our tools seem natural for the development and study of new numerical algorithms, extending ideas arising from Lie group retraction mappings, already in application when one discretizes objects in the category of Lie groups.

However the aim of this paper was not to open new questions in the area mentioned above, the present work naturally arose as a first step in a larger study of the discretization of variational formulations of field theories that admit symmetries and of its reduction. The focus here was on forward difference operators as main tool used for the discretization of a Lagrangian density that admits symmetries, or the discretization of a reduced lagrangian density, defined on the bundle of connections, preserving at the same time all additional symmetries that could lead to Noether currents. Our larger study has several natural steps ahead. The first one, developing variational principles for discrete (reduced or un-reduced) Lagrangians has already been performed in the companion work [12]. This companion paper represents a first application of the theory we present here, and achieves a construction of variational integrators for Euler-Poincare equations on field theories, with Noether current conservation properties. Further work based on these papers is already in progress. 
Again, as indicated above, our work, though centred on principal bundles, may be extended to other bundles with a free proper action of a Lie group. The restriction to principal bundles (gauge fields) and reduced (EulerPoincaré) equations must not be seen as a limitation of the tools to a particular case, but a benchmark to evaluate the capacities of our tools in the presence of a large (transitive) symmetry group acting freely. The same tools can be easily applied for free, non-transitive actions.

\section{Acknowledgements}

This work was partially supported by Fundação para a Ciência e a Tecnologia (Portuguese Foundation for Science and Technology) through the project UID/MAT/00297/2013 (Centro de Matemática e Apliçõs) and UID/MAT/04561/2013 (Centro de Matemática, Aplicao̧ões Fundamentais e Investigação Operacional of Universidade de Lisboa CMAF-CIO).

\section{References}

1. P.A. Absil, R. Mahony, and R. Sepulchre. Optimization algorithms on matrix manifolds. Princeton Univ. Press, Princeton, NJ, 2008

2. R.L. Adler, J.P. Dedieu, J.Y. Margulies, M. Martens, M. Shub. Newton's method on Riemannian manifolds and a geometric model for the human spine. IMA Journal of Numerical Analysis, 22(3), (2002) 359-390.

3. M. F. Atiyah, Complex analytic connections in fibre bundles. Transactions of the American Mathematical Society 85.1 (1957): 181207.

4. I. Biswas, F. Neumann. Atiyah sequences, connections and characteristic forms for principal bundles over groupoids and stacks. Comptes Rendus Mathematique 352.1 (2014): 59-64.

5. A.I. Bobenko, Yu.B. Suris. Discrete Lagrangian reduction, discrete Euler-Poincaré equations, and semidirect products. Letters in Mathematical Physics 49.1 (1999): 79-93.

6. R.D. Bos. Continuous representations of groupoids. Houston J. Math 37.3 (2011): 807-844.

7. R.D. Bos. Groupoids in geometric quantization. PhD Thesis Radboud University of Nijmegen, 2007.

8. N. Bou-Rabee, J.E. Marsden. Hamilton-Pontryagin integrators on Lie groups part I: Introduction and structure-preserving properties Foundations of Computational Mathematics 9.2 (2009): 197-219.

9. A. Cannas da Silva, A. Weinstein. Geometric models for noncommutative algebras. Vol. 10. American Mathematical Soc., 1999.

10. A.C. Casimiro, C. Rodrigo, First variation formula and conservation laws in several independent discrete variables. J. Geom. Phys. 62.1 (2012), 61-86.

11. A.C. Casimiro, C. Rodrigo, First variation formula for discrete variational problems in two independent variables. RACSAM Rev. R. Acad. A 106.1 (2012), 111-135.

12. A.C. Casimiro, C. Rodrigo, Variational Integrators for reduced field equations, Statistics Opt. Inform. Comput., Vol. 6, (2018),86-115.

13. M. Castrillón López, Field theories: reduction, constraints and variational integrators. Revista de la Real Academia de Ciencias Exactas, Físicas y Naturales. Serie A. Matematicas 106(1) (2012): 67-74

14. M. Castrillón López, P.L. García, T.S. Ratiu, Euler-Poincaré reduction on principal bundles, Lett. Math. Phys. 58 (2001) 167-180

15. M. Castrillón López, P.L. García, C. Rodrigo, Euler-Poincaré reduction in principal bundles by a subgroup of the structure group, Journal of Geometry and Physics, Volume 74, December 2013, Pages 352-369

16. K. Crane, Discrete connections for geometry processing. Diss. California Institute of Technology, 2010.

17. P.E. Crouch, R. Grossman. Numerical integration of ordinary differential equations on manifolds. Journal of Nonlinear Science 3.1 (1993): 1-33.

18. F. Demoures, F. Gay-Balmaz, S. Leyendecker, S. Ober-Bl?baum, T.S. Ratiu, T. S., Y. Weinand. Discrete variational Lie group formulation of geometrically exact beam dynamics. Numerische Mathematik, 130(1), (2015) 73-123.

19. F. Demoures, F. Gay-Balmaz, T.S. Ratiu. Multisymplectic variational integrators and space/time symplecticity. Analysis and Applications, 14(03), (2016) 341-391.

20. A. Edelman, T.A. Arias, S.T. Smith. The geometry of algorithms with orthogonality constraints. SIAM journal on Matrix Analysis and Applications 20.2 (1998): 303-353.

21. C. Ehresmann, Les connexions infinitésimales dans un espace fibré différentiable, Coll. de Topologie, Bruxelles, CBRM (1950), $29-55$.

22. D.C.P. Ellis, F. Gay-Balmaz, D.D. Holm, V. Putkaradze, T.S. Ratiu, Symmetry reduced dynamics of charged molecular strands, Arch. Rat. Mech. and Anal., 197(2), 811-902 (2010)

23. D.C.P. Ellis, F. Gay-Balmaz, D.D. Holm, T.S. Ratiu, Lagrange-Poincaré field equations, J. Geom. Phys. 61 (11) (2011) $2120-2146$.

24. J. Fernandez, M. Zuccalli. A geometric approach to discrete connections on principal bundles. Journal of Geometric Mechanics . Dec2013, Vol. 5 Issue 4, p433-444. 12p.

25. E.S. Gawlik, P. Mullen, D. Pavlov, J.E. Marsden, M. Desbrun. Geometric, variational discretization of continuum theories. Physica D: Nonlinear Phenomena, 240(21), (2011) 1724-1760.

26. A. Iserles, H.Z. Munthe-Kaas, S. P. N?rsett, A. Zanna. Lie-group methods. Acta Numerica, (2005) 1-148.

27. F. Jiménez, M. Kobilarov, D. Martín de Diego. Discrete variational optimal control. J. of Nonlinear Sci. 23.3 (2013): $393-426$. 
28. M. B. Kobilarov, J. E. Marsden. Discrete geometric optimal control on Lie groups IEEE Transactions on Robotics 27.4 (2011): 641-655.

29. M. Kobilarov. Solvability of Geometric Integrators for Multi-body Systems. Multibody Dynamics. Springer International Publishing, 2014. $145-174$.

30. A. Kock. Synthetic geometry of manifolds. Vol. 180. Cambridge University Press, 2010.

31. R. McLachlan, M. Perlmutter. Integrators for nonholonomic mechanical systems Journal of nonlinear science 16.4 (2006): 283-328.

32. M. Leok, J.E. Marsden, and A.D. Weinstein. A discrete theory of connections on principal bundles. arXiv preprint math/0508338 (2005).

33. J.E. Marsden, S. Pekarsky, S. Shkoller. Symmetry reduction of discrete Lagrangian mechanics on Lie groups. Journal of geometry and physics 36.1 (2000): 140-151.

34. J.E. Marsden, J. Scheurle. The reduced Euler-Lagrange equations. Fields Institute Comm 1 (1993): 139-164.

35. A. Saccon. Midpoint rule for variational integrators on Lie groups. International journal for numerical methods in engineering 78.11 (2009): 1345.

36. D.J. Saunders. The geometry of jet bundles. Vol. 142. Cambridge University Press, 1989.

37. J.P. Serre, Lie algebras and Lie groups, Lectures given at Harvard University, 1964, Benjamin, New York, 1965.

38. M. Shub. Some Remarks on Dynamical Systems and Numerical Analysis, in: Dynamical Systems and Partial Differential Equations, Proceedings of VII ELAM (L. Lara-Carrero and J. Lewowicz eds.), Equinoccio, Universidad Simon Bolivar, Caracas, (1986), 69-92

39. J. Vankerschaver. Euler-Poincaré reduction for discrete field theories. Journal of mathematical physics, 48(3), (2007) 032902.

40. J. Vankerschaver, F. Cantrijn. Discrete Lagrangian field theories on Lie groupoids. Journal of Geometry and Physics 57(2) (2007): 665-689. 